\title{
1,6-Stannatropic Strategy : Effective Generation and Cyclization of 1,5-Dipoles from o-Stannylmethylated Thioanilides or Phenyl Isothiocyanates
}

Satoshi Minakata,* Yukihiro Kasano, Hirofumi Ota, Yoji Oderaotoshi, and Mitsuo Komatsu*

\section{Contents}

S1 General Methods

S2-S19 Experimental Procedure and Spectral Data

S20 Time Course of Thermal Reaction of Thioanilide 1a

S21-S76 ${ }^{1} \mathrm{H}$ and ${ }^{13} \mathrm{C}$ NMR Spectra of Compound 1a-h, 2a-h, 3a-d, 4a, 5a-d, 6-8

General Methods.: Melting points were determined on a Yanaco micro melting point apparatus and are uncorrected. IR spectra were obtained on a Jasco FT/IR-410 infrared spectrophotometer. $\quad{ }^{1} \mathrm{H}$ and ${ }^{13} \mathrm{C}$-NMR spectra were recorded on a JEOL FTNMR JNM EX 270 spectrometer ( $\left.{ }^{1} \mathrm{H}-\mathrm{NMR}, 270 \mathrm{MHz} ;{ }^{13} \mathrm{C}-\mathrm{NMR}, 68 \mathrm{MHz}\right)$ using tetramethylsilane as an internal standard. Mass spectra and high-resolution mass spectral data were obtained on a JEOL DX-303 mass spectrometer. Products were purified by chromatography on silica gel BW-300 (Fuji Silysia Chemical Co.). Preparative gel permeation liquid chromatography (GPLC) was performed on a JAI (Japan Analytical Industry) LC-908 instrument with JAIGEL 1H-2H columns and 
chloroform as an eluent. Analytical thin layer chromatography was performed using EM reagent $0.25 \mathrm{~mm}$ silica gel glass plates (silica gel $60 \mathrm{~F} 254,0.25 \mathrm{~mm}$ thickness) (MERCK Co.). Visualization was accomplished with UV light and ethanolic phosphomolybdic acid followed by heating. All reactions were carried out under an atmosphere of nitrogen. Organic solvents were dried and distilled prior to use.

\section{Experimental Procedure and Spectral Data}

2-Benzylbenzenamine, 2-benzyl-4-chlorobenzenamine, 2-benzyl-4-methoxybenzenamine, and 2-(4-chlorophenylmethyl)benzenamine were produced by reduction of the corresponding ketones. ${ }^{1}$

General procedure of preparation of $o$-tributylstannylmethylated aniline derivatives n-Butyllithium (1.6 M hexane solution, $7.5 \mathrm{~mL}, 12 \mathrm{mmol}$ ) was slowly added to a THF solution (50 $\mathrm{mL}$ ) of a 2-benzylbenzenamine derivative (10 mmol) over $5 \mathrm{~min}$ at $78{ }^{\circ} \mathrm{C}$, and was stirred for $1 \mathrm{~h}$ at $-78{ }^{\circ} \mathrm{C}$. To the solution was added trimethylsilyl chloride (1.5 mL, $12 \mathrm{mmol})$ over $5 \mathrm{~min}$. After the solution was stirred for $1 \mathrm{~h}$ at $-78{ }^{\circ} \mathrm{C}$, sec-butyllithium (1.3 M cyclohexane solution, $11.5 \mathrm{~mL}, 15 \mathrm{mmol}$ ) was added over 10 min. Then, the reaction mixture was stirred for $1 \mathrm{~h}$ at $-78{ }^{\circ} \mathrm{C}$, and tributylstannyl chloride (7.3 mL, $27 \mathrm{mmol})$ was added. The reaction mixture was warmed to room temperature, and was stirred for $1 \mathrm{~h}$. After the solvents were removed from the mixture in vacuo, diethyl ether $(50 \mathrm{~mL})$ was added to the residue. The solution was washed with water $(50 \mathrm{~mL})$, and was dried over anhydrous potassium carbonate. The organic layer was concentrated and purified by silica gel column chromatography (eluent: hexane/ethyl acetate) to give the corresponding o-tributylstannylmethylated 
aniline derivative.

2-( $\alpha$-Tributylstannylbenzyl)benzenamine: 89\% yield; yellow oil; IR (neat) $v_{\max } 3435$, 2956, 2924, 1618, 1491, 1452, $1271 \mathrm{~cm}^{-1} ;{ }^{1} \mathrm{H}-\mathrm{NMR}\left(\mathrm{CDCl}_{3}, 270 \mathrm{MHz}\right) \delta$ 0.71-0.95 (m, 15H), 1.15-1.46 (m, 12H), 3.46 (brs, 2H), 4.00 (t, 1H, $\left.{ }^{2} J_{\mathrm{H}-\mathrm{Sn}}=35 \mathrm{~Hz}\right), 6.67-6.81(\mathrm{~m}, 2 \mathrm{H})$, 7.00-7.47 (m, 7H); ${ }^{13} \mathrm{C}-\mathrm{NMR}\left(\mathrm{CDCl}_{3}, 68 \mathrm{MHz}\right) \delta 11.2,13.7,27.4,29.0,38.2,116.3$, 118.9, 124.0, 126.4, 128.4, 128.5, 131.2, 144.0, 144.5; EI-MS m/z $473\left(\mathrm{M}^{+}, 3\right), 416\left(\mathrm{M}^{+}\right.$ - $\mathrm{Bu}, 100), 182\left(\mathrm{M}^{+}-\mathrm{Bu}_{3} \mathrm{Sn}, 83\right)$; Anal. Calcd for $\mathrm{C}_{25} \mathrm{H}_{39} \mathrm{NSn}$ : C, 63.58; H, 8.32; N, 2.97. Found: C, 63.46; H, 8.40; N, 2.96.

4-Chloro-2-( $\alpha$-tributylstannylbenzyl)benzenamine: $80 \%$ yield; yellow oil; IR (neat) $v_{\max }$ 3439, 2956, 2924, 1620, 1491, 1279, 1074, 806, 752, $700 \mathrm{~cm}^{-1} ;{ }^{1} \mathrm{H}-\mathrm{NMR}\left(\mathrm{CDCl}_{3}, 270\right.$ MHz) $\delta$ 0.71-0.95 (m, 15H), 1.16-1.49 (m, 12H), 3.44 (brs, 2H), 3.95 (t, 1H, ${ }^{2} J_{\mathrm{H}-\mathrm{Sn}}=35$ $\mathrm{Hz}$ ), 6.60 (d, 1H, $J=8.4 \mathrm{~Hz}), 6.96-7.08$ (m, 5H), 7.19-7.25 (m, 2H); ${ }^{13} \mathrm{C}-\mathrm{NMR}\left(\mathrm{CDCl}_{3}\right.$, $68 \mathrm{MHz}) \delta 11.4,13.7,27.4,29.0,38.3,117.3,123.4,124.3,126.0,126.6,128.6,130.3$, 130.6, 142.87, 142.93; EI-MS m/z 507 (M+, 2), 450 (M+ $\left.\mathrm{M}^{+}-\mathrm{Bu}, 100\right), 291\left(\mathrm{Bu}_{3} \mathrm{Sn}^{+}, 50\right)$, $216\left(\mathrm{M}^{+}-\mathrm{Bu}_{3} \mathrm{Sn}, 80\right)$; Anal. Calcd for $\mathrm{C}_{25} \mathrm{H}_{38} \mathrm{ClNSn}$ : C, 59.25; H, 7.56; N, 2.76. Found: C, 59.14 H, 7.67; N, 2.83.

4-Methoxy-2-( $\alpha$-tributylstannylbenzyl)benzenamine: 73\% yield; yellow oil; IR (neat) $v_{\max } 3419,2924,1601,1498,1460,1246,1043,806,758,700 \mathrm{~cm}^{-1} ;{ }^{1} \mathrm{H}-\mathrm{NMR}\left(\mathrm{CDCl}_{3}\right.$, $270 \mathrm{MHz}) \delta$ 0.70-0.95 (m, 15H), 1.15-1.44 (m, 12H), 3.77 (brs, 2H), 3.75 (s, 3H), 4.01 $\left(\mathrm{t}, 1 \mathrm{H},{ }^{2} J_{\mathrm{H}-\mathrm{Sn}}=35 \mathrm{~Hz}\right), 6.62-6.71(\mathrm{~m}, 3 \mathrm{H}), 6.99-7.05(\mathrm{~m}, 3 \mathrm{H}), 7.17-7.24(\mathrm{~m}, 2 \mathrm{H}) ;{ }^{13} \mathrm{C}-$ NMR $\left(\mathrm{CDCl}_{3}, 68 \mathrm{MHz}\right) \delta$ 11.4, 13.7, 27.4, 29.0, 38.8, 55.7, 111.4, 116.7, 117.5, 124.0, 
126.5, 128.5, 130.7, 137.9, 143.7, 153.0; CI-MS m/z $504\left(\mathrm{MH}^{+}, 22\right), 446\left(\mathrm{M}^{+}-\mathrm{Bu}, 94\right)$, $291\left(\mathrm{Bu}_{3} \mathrm{Sn}^{+}, 42\right), 212\left(\mathrm{M}^{+}-\mathrm{Bu}_{3} \mathrm{Sn}, 20\right), 87$ (100); Anal. Calcd for $\mathrm{C}_{26} \mathrm{H}_{41} \mathrm{NOSn}$ : C, 62.17; H, 8.23; N, 2.79. Found: C, 61.89; H, 8.21; N, 2.87.

2-[(4-Chlorophenyl)-tributylstannylmethyl]benzenamine: yellow oil; 78\% yield; IR (neat) $v_{\max } 3439,2924,1618,1487,1456,1375,1281,1171,1092,1011,960,829,750$ $\mathrm{cm}^{-1} ;{ }^{1} \mathrm{H}-\mathrm{NMR}\left(\mathrm{CDCl}_{3}, 270 \mathrm{MHz}\right) \delta$ 0.71-0.95 (m, 15H), 1.16-1.42 (m, 12H), 3.43 (brs, 2H), $3.94\left(\mathrm{t}, 1 \mathrm{H},{ }^{2} \mathrm{~J}_{\mathrm{H}-\mathrm{Sn}}=35 \mathrm{~Hz}\right), 6.68-6.81(\mathrm{~m}, 2 \mathrm{H}), 6.92-7.18(\mathrm{~m}, 6 \mathrm{H}) ;{ }^{13} \mathrm{C}-\mathrm{NMR}$ $\left(\mathrm{CDCl}_{3}, 68 \mathrm{MHz}\right) \delta 11.2,13.7,27.4,29.0,37.5,116.5,119.0,126.7,127.6,128.0,128.5$, 129.4, 131.3, 142.8, 144.4; EI-MS m/z $507\left(\mathrm{M}^{+}, 2\right), 450\left(\mathrm{M}^{+}-\mathrm{Bu}, 100\right), 291\left(\mathrm{Bu}_{3} \mathrm{Sn}^{+}\right.$, 49), $216\left(\mathrm{M}^{+}-\mathrm{Bu}_{3} \mathrm{Sn}, 86\right)$; Anal. Calcd for $\mathrm{C}_{25} \mathrm{H}_{38} \mathrm{ClNSn}$ : C, 59.25; H, 7.56; N, 2.76 . Found: C, 59.10; H, 7.38; N, 2.76.

Preparation of a $o$-tributylstannylmethylated aniline derivative n-Butyllithium (1.6 M hexane solution, $7.5 \mathrm{~mL}, 12 \mathrm{mmol}$ ) was slowly added to a THF solution $(50 \mathrm{ml})$ of $o$-toluidine $(1.1 \mathrm{~mL}, 10 \mathrm{mmol})$ over $5 \mathrm{~min}$ at $-78{ }^{\circ} \mathrm{C}$, and was stirred for $1 \mathrm{~h}$ at $-78^{\circ} \mathrm{C}$. To the solution was added trimethylsilyl chloride (1.5 mL, 12 mmol) over $5 \mathrm{~min}$, and the mixture was stirred for $1 \mathrm{~h}$ at room temperature. THF was removed from the solution under reduced pressure, and to the residue was added hexane (50 mL). $\quad n$-Butyllithium (1.6 M hexane solution, $7.5 \mathrm{~mL}, 12 \mathrm{mmol}$ ) was added to the mixture over $5 \mathrm{~min}$ at $0{ }^{\circ} \mathrm{C}$ and was stirred for $1 \mathrm{~h}$. sec-Butyllithium (1.3 $\mathrm{M}$ cyclohexane solution, $11.5 \mathrm{~mL}, 12 \mathrm{mmol}$ ) was added to the solution over $10 \mathrm{~min}$ at $0{ }^{\circ} \mathrm{C}$ and was refluxed for $6 \mathrm{~h}$. To the solution was added tributylstannyl chloride (7.3 mL, $27 \mathrm{mmol}$ ) at room temperature and the mixture was stirred for $1 \mathrm{~h}$. After the solvent 
was removed from the mixture in vacuo, diethyl ether $(50 \mathrm{ml})$ was added to the residue. The solution was washed with water $(50 \mathrm{ml})$ and dried over potassium carbonate. The organic layer was concentrated and purified by silica gel column chromatography (eluent: hexane / ethyl acetate) and gel permeation column chromatography (JAIGEL2H, Japan Analytical Industry, eluent: chloroform) to give 2(tributylstannylmethyl)benzenamine (1.35 g, 34\%).

2-Tributylstannylmethylbenzenamine: orange colored oil; IR (neat) $v_{\max } 2924,1620$, 1495, 1456, 1076, 864, $743 \mathrm{~cm}^{-1} ;{ }^{1} \mathrm{H}-\mathrm{NMR}\left(\mathrm{CDCl}_{3}, 270 \mathrm{MHz}\right) \delta$ 0.69-0.95 (m, 15H), 1.18-1.48 (m, 12H), $2.11\left(\mathrm{t}, 2 \mathrm{H},{ }^{2} J_{\mathrm{H}-\mathrm{Sn}}=27 \mathrm{~Hz}\right), 3.41$ (brs, 2H), 6.60-6.68 (m, 2H), 6.82-6.92 (m, 2H); ${ }^{13} \mathrm{C}-\mathrm{NMR}\left(\mathrm{CDCl}_{3}, 68 \mathrm{MHz}\right) \delta 10.1$, 13.8, 13.9, 27.4, 29.1, 115.0, 118.9, 124.2, 128.0, 128.2, 142.0; Anal. Calcd for $\mathrm{C}_{19} \mathrm{H}_{35} \mathrm{NSn}$ : C, 57.60; H, 8.90; N, 3.54. Found: C, 57.42 H, 8.83; N, 3.64.

General procedure of preparation of $o$-tributylstannylmethylated thioanilide derivatives

An acid chloride (2 mmol) was added to a THF solution $(5 \mathrm{~mL})$ of an $o^{-}$ tributylstannylmethylated aniline derivative and triethylamine $(0.279 \mathrm{ml}, 2 \mathrm{mmol})$ at 0 ${ }^{\circ} \mathrm{C}$, and was stirred for $3 \mathrm{~h}$ at room temperature. The solvent was removed from the solution and diethyl ether (30 mL) was added to the residue. The mixture was washed with a saturated ammonium chloride aqueous solution $(10 \mathrm{~mL})$ and dried over potassium carbonate. The organic layer was concentrated and was purified by silica gel column chromatography (eluent: hexane/ethyl acetate) to give the corresponding $o$ tributylstannylmethylated anilide derivative.

A benzene solution $(15 \mathrm{~mL})$ of an $o$-tributylstannylmethylated anilide 
derivative (2 mmol) with Lawesson's reagent $(0.61 \mathrm{~g}, 1.5 \mathrm{mmol})$ was heated at $60{ }^{\circ} \mathrm{C}$ for $3 \mathrm{~h}$. The solution was cooled to room temperature and the white precipitate was removed by filtration. After the filtrate was concentrated the residue was purified by silica gel column chromatography (eluent: hexane/ethyl acetate) to give the corresponding o-tributylstannylmethylated thioanilide derivative.

$N$-[2-( $\alpha$-Tributylstannylbenzyl)phenyl]benzamide: 96\% yield; colorless solid; mp 52-53 ${ }^{\circ} \mathrm{C}$; IR (KBr) $v_{\max }$ 2954, 2925, $1666(\mathrm{C}=\mathrm{O}), 1521,1450,1303,752 \mathrm{~cm}^{-1}$; ${ }^{1} \mathrm{H}-\mathrm{NMR}$ $\left(\mathrm{CDCl}_{3}, 270 \mathrm{MHz}\right) \delta 0.77-0.86(\mathrm{~m}, 15 \mathrm{H}), 1.11-1.41(\mathrm{~m}, 12 \mathrm{H}), 4.08\left(\mathrm{t}, 1 \mathrm{H},{ }^{2} J_{\mathrm{H}-\mathrm{Sn}}=34\right.$ Hz), 7.04-7.49 (m, 13H), 7.73 (brs, $1 \mathrm{H}), 8.19$ (d, 1H, $J=7.3 \mathrm{~Hz}) ;{ }^{13} \mathrm{C}-\mathrm{NMR}\left(\mathrm{CDCl}_{3}, 68\right.$ MHz) $\delta 10.7,13.6,27.3,28.8,38.3,123.0,124.4,124.9,126.7,128.4,128.5,129.0$, 131.5, 131.7, 133.3, 134.7, 136.5, 143.4, 164.8; EI-MS m/z $577\left(\mathrm{M}^{+}, 34\right), 520\left(\mathrm{M}^{+}-\mathrm{Bu}\right.$, 83), $105\left(\mathrm{PhCO}^{+}, 100\right)$; Anal. Calcd for $\mathrm{C}_{32} \mathrm{H}_{43} \mathrm{NOSn}$ : C, 66.68; H, 7.52; N, 2.43. Found: C, 66.42; H, 7.42; N, 2.42.

$N$-[2-( $\alpha$-Tributylstannylbenzyl)phenyl]thiobenzamide (1a): 95\% yield; yellow solid; mp 76-77 ${ }^{\circ} \mathrm{C}$; IR (KBr) $v_{\max }$ 3255, 2927, 1506, 1486, 1448, $1358(\mathrm{C}=\mathrm{S}), 1213,698 \mathrm{~cm}^{-1}$; ${ }^{1} \mathrm{H}-\mathrm{NMR}\left(\mathrm{CDCl}_{3}, 270 \mathrm{MHz}\right) \delta$ 0.78-0.97 (m, 15H), 1.13-1.41 (m, 12H), $4.12\left(\mathrm{t}, 1 \mathrm{H},{ }^{2} J_{\mathrm{H}-}\right.$ $\mathrm{Sn}=33 \mathrm{~Hz}), 6.98-7.45(\mathrm{~m}, 11 \mathrm{H}), 7.64(\mathrm{~d}, 2 \mathrm{H}, J=7.3 \mathrm{~Hz}), 7.89(\mathrm{~d}, 1 \mathrm{H}, J=6.8 \mathrm{~Hz}), 8.71$ (brs, $1 \mathrm{H}) ;{ }^{13} \mathrm{C}-\mathrm{NMR}\left(\mathrm{CDCl}_{3}, 68 \mathrm{MHz}\right) \delta 10.8,13.7,27.4,29.0,37.5,124.4,126.1$, 126.5, 126.6, 126.9, 127.3, 128.3, 128.7, 131.1, 132.1, 137.7, 138.6, 141.8, 143.9, 197.9; CI-MS m/z $594\left(\mathrm{MH}^{+}, 40\right), 302\left(\mathrm{M}^{+}-\mathrm{Bu}_{3} \mathrm{Sn}, 92\right), 270\left(\mathrm{M}^{+}-\mathrm{Bu}_{3} \mathrm{Sn}-\mathrm{S}, 100\right)$; Anal. Calcd for $\mathrm{C}_{32} \mathrm{H}_{43} \mathrm{NSSn}$ : C, 64.87; H, 7.32; N, 2.36. Found: C, 64.76; H, 7.25; N, 2.31 . 
$N$-[2-( $\alpha$-Tributylstannylbenzyl)phenyl]-4-methoxybenzamide: $88 \%$ yield; colorless solid; mp 45-47 ${ }^{\circ} \mathrm{C}$; IR (KBr) $v_{\max }$ 3352, 2956, 1643 (C=S), 1502, $1253 \mathrm{~cm}^{-1}$; ${ }^{1} \mathrm{H}-\mathrm{NMR}$ $\left(\mathrm{CDCl}_{3}, 270 \mathrm{MHz}\right) \delta$ 0.71-0.95 (m, 15H), 1.11-1.39 (m, 12H), 3.83 (s, 3H), $4.08(\mathrm{t}, 1 \mathrm{H}$, ${ }^{2} J_{\mathrm{H}-\mathrm{Sn}}=35 \mathrm{~Hz}$ ), 6.81 (d, 2H, $J=8.9 \mathrm{~Hz}$ ), 7.04-7.35 (m, 10H), 7.64 (brs, 1H), 8.16 (d, $1 \mathrm{H}, J=7.3 \mathrm{~Hz}) ;{ }^{13} \mathrm{C}-\mathrm{NMR}\left(\mathrm{CDCl}_{3}, 68 \mathrm{MHz}\right) \delta 10.7,13.6,27.4,29.0,38.3,55.4,113.4$, 122.9, 124.2, 124.9, 126.7, 126.8, 127.0, 128.7, 129.0, 131.7, 133.1, 136.7, 143.5, 162.1, 164.3; EI-MS m/z 607 (M+, 37), 550 (M+ - Bu, 88), 135 (4- $\mathrm{CH}_{3} \mathrm{OC}_{6} \mathrm{H}_{4} \mathrm{CO}^{+}$, 100); Anal. Calcd for $\mathrm{C}_{33} \mathrm{H}_{45} \mathrm{NO}_{2} \mathrm{Sn}$ : C, 65.36; H, 7.48; N, 2.31. Found: C, 65.28; H, 7.36; N, 2.28. $N$-[2-( $\alpha$-Tributylstannylbenzyl)phenyl]-4-methoxythiobenzamide $\quad(\mathbf{1 b}): \quad 98 \% \quad$ yield; yellow solid; mp 85-88 ${ }^{\circ} \mathrm{C}$; IR (KBr) $v_{\max }$ 3216, 2927, 1606, 1498, 1352 (C=S), 1245, $1182 \mathrm{~cm}^{-1} ;{ }^{1} \mathrm{H}-\mathrm{NMR}\left(\mathrm{CDCl}_{3}, 270 \mathrm{MHz}\right) \delta$ 0.73-0.97 (m, 15H), 1.13-1.41 (m, 12H), 3.83 (s, 3H), $4.11\left(\mathrm{t}, 1 \mathrm{H},{ }^{2} J_{\mathrm{H}-\mathrm{Sn}}=33 \mathrm{~Hz}\right), 6.79-6.82$ (m, 2H), 6.97-7.09 (m, 3H), 7.18-7.37 (m, 5H), 7.60-7.62 (m, 2H), 7.85 (brs, $1 \mathrm{H}), 8.63$ (brs 1H); ${ }^{13} \mathrm{C}-\mathrm{NMR}\left(\mathrm{CDCl}_{3}, 68 \mathrm{MHz}\right) \delta$ 10.8, 13.7, 27.3, 29.0, 37.5, 55.5, 113.4, 124.4, 126.1, 126.5, 126.97, 127.04, 128.5, 128.7, 132.0, 134.0, 137.9, 138.4, 143.9, 162.1, 196.6; EI-MS m/z $624\left(\mathrm{MH}^{+}, 35\right), 566$ $\left(\mathrm{M}^{+}-\mathrm{Bu}, 14\right), 332\left(\mathrm{M}^{+}-\mathrm{Bu}_{3} \mathrm{Sn}, 68\right), 300\left(\mathrm{M}^{+}-\mathrm{Bu}_{3} \mathrm{Sn}-\mathrm{S}, 100\right)$; Anal. Calcd for $\mathrm{C}_{33} \mathrm{H}_{45} \mathrm{NOSSn}$ : C, 63.67; H, 7.29; N, 2.25. Found: C, 63.58; H, 7.11; N, 2.27.

$N$-[2-( $\alpha$-Tributylstannylbenzyl)phenyl]-4-trifluoromethylbenzamide: $96 \%$ yield; pale yellow solid; mp 65-67 ${ }^{\circ} \mathrm{C}$; IR (KBr) $v_{\max }$ 3315, 2925, 1649 (C=O), 1531, 1502, 1332, 1128, $1068 \mathrm{~cm}^{-1}$; ${ }^{1} \mathrm{H}-\mathrm{NMR}\left(\mathrm{CDCl}_{3}, 270 \mathrm{MHz}\right) \delta$ 0.71-0.95 (m, 15H), 1.02-1.45 (m, $12 \mathrm{H}), 4.07$ (t, $1 \mathrm{H},{ }^{2} J_{\mathrm{H}-\mathrm{Sn}}=34 \mathrm{~Hz}$ ), $7.00-7.76$ (m, 12H), 7.88 (brs, $\left.1 \mathrm{H}\right), 8.20$ (d, $1 \mathrm{H}, J=$ 8.1 Hz); ${ }^{13} \mathrm{C}-\mathrm{NMR}\left(\mathrm{CDCl}_{3}, 68 \mathrm{MHz}\right) \delta 10.7,13.6,27.3,28.9,38.3,122.7,123.5$ (q, $J=$ $272 \mathrm{~Hz}), 124.8,125.1,125.3,125.4,125.5,126.7,127.2,131.8,132.3$ (q, $J=32 \mathrm{~Hz})$, 
133.2, 136.2, 138.0, 143.2, 163.3; EI-MS m/z $645\left(\mathrm{M}^{+}\right.$, 78), $588\left(\mathrm{M}^{+}-\mathrm{Bu}, 100\right), 291$ $\left(\mathrm{Bu}_{3} \mathrm{Sn}^{+}, 72\right), 173\left(4-\mathrm{CF}_{3} \mathrm{C}_{6} \mathrm{H}_{4} \mathrm{CO}^{+}\right.$, 69); Anal. Calcd for $\mathrm{C}_{33} \mathrm{H}_{42} \mathrm{~F}_{3} \mathrm{NOSn}$ : C, 61.51; $\mathrm{H}$, 6.57; N, 2.17. Found: C, 61.50; H, 6.63; N, 2.18.

$N$-[2-( $\alpha$-Tributylstannylbenzyl)phenyl]-4-trifluoromethylthiobenzamide $\quad$ (1c): $\quad 90 \%$ yield; yellow solid; mp 86-88 ${ }^{\circ} \mathrm{C}$; IR (KBr) $v_{\max }$ 3218, 2929, 1491, 1325 (C=S), 1164, 1128, $1068 \mathrm{~cm}^{-1}$; ${ }^{1} \mathrm{H}-\mathrm{NMR}\left(\mathrm{CDCl}_{3}, 270 \mathrm{MHz}\right) \delta$ 0.73-0.97 (m, 15H), 1.13-1.43 (m,12H), $4.08\left(\mathrm{t}, 1 \mathrm{H},{ }^{2} J_{\mathrm{H}-\mathrm{Sn}}=33 \mathrm{~Hz}\right), 6.95-7.37(\mathrm{~m}, 8 \mathrm{H}), 7.56-7.67(\mathrm{~m}, 4 \mathrm{H}), 7.95$ (d, $1 \mathrm{H}, J=6.5$ $\mathrm{Hz}), 8.74$ (brs, $1 \mathrm{H}) ;{ }^{13} \mathrm{C}-\mathrm{NMR}\left(\mathrm{CDCl}_{3}, 68 \mathrm{MHz}\right) \delta 10.7,13.5,27.2,28.9,37.5,123.5$ (q, $J=272 \mathrm{~Hz}), 124.4,125.1,126.1,126.3,126.4,126.8,127.4,128.7,132.1,132.3$ (q, $J=$ $33 \mathrm{~Hz}), 137.3,138.2,143.6,144.6,195.8$; EI-MS m/z $661\left(\mathrm{M}^{+}, 1\right), 604\left(\mathrm{M}^{+}\right.$- Bu, 7), 370 $\left(\mathrm{M}^{+}-\mathrm{Bu}_{3} \mathrm{Sn}, 100\right), 338\left(\mathrm{M}^{+}-\mathrm{Bu}_{3} \mathrm{Sn}-\mathrm{S}, 88\right)$; Anal. Calcd for $\mathrm{C}_{33} \mathrm{H}_{42} \mathrm{~F}_{3} \mathrm{NSSn}$ : C, 60.01; H, 6.41; N, 2.12. Found: C, 59.96; H, 6.40; N, 2.21.

$N$-[2-( $\alpha$-Tributylstannylbenzyl)phenyl]cinnamamide: 76\% yield; colorless solid; mp 56$57{ }^{\circ} \mathrm{C}$; IR (KBr) $v_{\max } 3232,2954,1657(\mathrm{C}=\mathrm{O}), 1622,1535,1448,1344,1191,750 \mathrm{~cm}^{-1}$; ${ }^{1} \mathrm{H}-\mathrm{NMR}\left(\mathrm{CDCl}_{3}, 270 \mathrm{MHz}\right) \delta$ 0.78-1.00 (m, 15H), 1.15-1.42 (m, 12H), $4.10\left(\mathrm{t}, 1 \mathrm{H},{ }^{2} J_{\mathrm{H}-}\right.$ $\mathrm{Sn}=34 \mathrm{~Hz}), 6.22$ (d, 1H, $J=16 \mathrm{~Hz}), 7.06-7.50$ (m, 15H), 8.06 (brs, $1 \mathrm{H}) ;{ }^{13} \mathrm{C}-\mathrm{NMR}$ $\left(\mathrm{CDCl}_{3}, 68 \mathrm{MHz}\right) \delta 10.8,13.6,27.3,28.9,37.8,121.2,123.5,124.6,126.4,126.6,126.7$, 127.6, 128.6, 128.8, 129.6, 131.4, 134.5, 136.0, 141.2, 143.6, 163.5; CI-MS m/z 604 $\left(\mathrm{MH}^{+}, 100\right), 546\left(\mathrm{M}^{+}-\mathrm{Bu}, 46\right)$; Anal. Calcd for $\mathrm{C}_{34} \mathrm{H}_{45} \mathrm{NOSn}$ C, 67.79; H, 7.53; N, 2.33. Found: C, 67.70; H, 7.52; N, 2.25.

$N$-[2-( $\alpha$-Tributylstannylbenzyl)phenyl]thiocinnamamide (1d): 78\% yield; yellow solid; mp 104-105 ${ }^{\circ} \mathrm{C}$; IR (KBr) $v_{\max }$ 2937, 1637, 1392 (C=S), 1176, $756 \mathrm{~m}^{-1}$; ${ }^{1} \mathrm{H}-\mathrm{NMR}$ $\left(\mathrm{CDCl}_{3}, 270 \mathrm{MHz}, 45^{\circ} \mathrm{C}\right) \delta$ 0.76-0.93 (m, 15H), 1.05-1.32 (m, 12H), $4.06(\mathrm{t}, 0.53 \mathrm{H}$, 
$\left.{ }^{2} J_{\mathrm{H}-\mathrm{Sn}}=33 \mathrm{~Hz}\right), 4.14\left(\mathrm{t}, 0.47 \mathrm{H},{ }^{2} J_{\mathrm{H}-\mathrm{Sn}}=31 \mathrm{~Hz}\right), 6.73(\mathrm{~d}, 0.47 \mathrm{H}, J=15 \mathrm{~Hz}), 6.87(\mathrm{~d}$, 0.53H, $J=16 \mathrm{~Hz}$ ), 7.00-7.68 (m, 14.53H), 8.09 (d, 0.47H, $J=15 \mathrm{~Hz}), 8.68$ (brs, 0.53H), 9.30 (brs, 0.47H); ${ }^{13} \mathrm{C}-\mathrm{NMR}\left(\mathrm{CDCl}_{3}, 68 \mathrm{MHz}, 45{ }^{\circ} \mathrm{C}\right) \delta 10.2,10.3,13.8,13.9,27.36$, 27.43, 28.7, 28.9, 36.2, 36.6, 122.0, 124.1, 124.2, 126.0, 126.2, 127.1, 127.8, 127.9, 128.1, 128.2, 128.4, 128.5, 128.7, 129.9, 130.1, 131.5, 131.9, 134.0, 134.2, 135.1, 136.9, 138.5, 139.0, 141.6, 142.4, 143.8, 147.3, 193.4, 195.9; CI-MS m/z 619 (M+ $\left.\mathrm{M}^{+}, 58\right), 327$ $\left(\mathrm{M}^{+}-\mathrm{H}-\mathrm{Bu}_{3} \mathrm{Sn}, 81\right), 131$ (100); Anal. Calcd for $\mathrm{C}_{34} \mathrm{H}_{45} \mathrm{NSSn}$ : C, 66.02; H, 7.33; N, 2.26. Found: C, 65.85; H, 7.21; N, 2.25.

$N$-\{2-[4-Chlorophenyl(tributylstannyl)methyl]phenyl $\}$ benzamide: yield 70\%; colorless solid; mp 72-74 ${ }^{\circ} \mathrm{C}$; IR (KBr) $v_{\max }$ 3236, 2923, 1643 (C=O), 1483, 1315, 754, $713 \mathrm{~cm}^{-1}$; ${ }^{1} \mathrm{H}-\mathrm{NMR}\left(\mathrm{CDCl}_{3}, 270 \mathrm{MHz}\right) \delta$ 0.73-0.99 (m, 15H), 1.12-1.42 (m, 12H), $4.03\left(\mathrm{t}, 1 \mathrm{H},{ }^{2} J_{\mathrm{H}-}\right.$ Sn $=34 \mathrm{~Hz}$ ), 6.96-6.99 (m, 2H), 7.14-7.52 (m, 10H), 7.65 (brs, 1H), 8.11 (d, 1H, $J=8.1$ $\mathrm{Hz}) ;{ }^{13} \mathrm{C}-\mathrm{NMR}\left(\mathrm{CDCl}_{3}, 68 \mathrm{MHz}\right) \delta 10.7,13.6,27.3,28.9,37.3,123.5,124.7,126.7$, 126.8, 127.8, 128.5, 128.9, 130.2, 131.6, 131.7, 133.2, 134.6, 136.2, 142.3, 164.9; CIMS m/z $612\left(\mathrm{MH}^{+}, 66\right), 554\left(\mathrm{M}^{+}-\mathrm{Bu}, 24\right), 322$ (100), $291\left(\mathrm{Bu}_{3} \mathrm{Sn}^{+}, 32\right), 105\left(\mathrm{PhCO}^{+}\right.$, 14); Anal. Calcd for $\mathrm{C}_{32} \mathrm{H}_{42} \mathrm{ClNOSn}$ : C, 62.92; H, 6.93; N, 2.29. Found: C, 62.79; H, 6.79; N, 2.32 .

$N$-\{2-[4-Chlorophenyl(tributylstannyl)methyl]phenyl\}thiobenzamide (1e): 88\% yield; yellow solid; IR (KBr) v $v_{\max }$ 2926, 1487, $1348(\mathrm{C}=\mathrm{S}), 774 \mathrm{~cm}^{-1} ;{ }^{1} \mathrm{H}-\mathrm{NMR}\left(\mathrm{CDCl}_{3}, 270\right.$ MHz) $\delta$ 0.74-0.98 (m, 15H), 1.14-1.42 (m, 12H), 4.09 (t, 1H, $\left.{ }^{2} J_{\mathrm{H}-\mathrm{Sn}}=32 \mathrm{~Hz}\right), 6.92$ (d, 2H, $J=8.4 \mathrm{~Hz}$ ), 7.15 (d, 2H, $J=8.4 \mathrm{~Hz}$ ), 7.34-7.51 (m, 6H), 7.67-7.74 (m, 3H), 8.62 (brs, 1H); ${ }^{13} \mathrm{C}-\mathrm{NMR}\left(\mathrm{CDCl}_{3}, 68 \mathrm{MHz}\right) \delta 10.9,13.7,27.4,29.0,36.8,126.4,126.5$, 127.3, 127.4, 127.6, 128.4, 128.6, 129.7, 131.3, 132.0, 137.6, 138.7, 141.7, 142.9, 
198.5; CI-MS m/z 628 (MH+, 18), 338 (100), 304 ( $\mathrm{M}^{+}-\mathrm{Bu}_{3} \mathrm{Sn}$ - S, 87); Anal. Calcd for $\mathrm{C}_{32} \mathrm{H}_{42}$ ClNSSn: C, 61.31; H, 6.75; N, 2.23. Found: C, 61.17; H, 6.59; N, 2.44.

$N$-[2-( $\alpha$-Tributylstannylbenzyl)phenyl]formamide: 53\% yield: yellow oil; IR (neat) $v_{\max }$ 3251, 2956, 1689 (C=O), 1596, 1492, 1294, $752 \mathrm{~cm}^{-1} ;{ }^{1} \mathrm{H}-\mathrm{NMR}\left(\mathrm{CDCl}_{3}, 270 \mathrm{MHz}\right) \delta$ 0.74-0.98 (m, 15H), 1.15-1.46 (m, 12H), $4.03\left(\mathrm{t}, 0.35 \mathrm{H},{ }^{2} J_{\mathrm{H}-\mathrm{Sn}}=34 \mathrm{~Hz}\right), 4.07$ (t, 0.65H, ${ }^{2} J_{\mathrm{H}-\mathrm{Sn}}=33 \mathrm{~Hz}$ ), 6.89 (brs, 0.35H), 6.89-7.32 (m, 9.3H), 7.98-8.01 (m, 0.35H), 8.262 and 8.268 (s x 2, 0.35H), 8.40 (s, 0.33H), 8.44 (s, 0.33H); ${ }^{13} \mathrm{C}-\mathrm{NMR}\left(\mathrm{CDCl}_{3}, 68 \mathrm{MHz}\right) \delta$ 10.9, 11.0, 13.7, 27.1, 28.9, 37.0, 37.4, 122.2, 123.8, 124.6, 125.0, 126.1, 126.3, 126.4, 128.4, 128.5, 128.7, 128.8, 131.7, 132.0, 133.5, 134.6, 134.8, 135.8, 143.0, 143.4, 158.7, 162.6; CI-MS m/z $501\left(\mathrm{M}^{+}, 100\right), 444\left(\mathrm{M}^{+}\right.$- Bu, 86), $291\left(\mathrm{Bu}_{3} \mathrm{Sn}^{+}, 61\right)$; Anal. Calcd for $\mathrm{C}_{26} \mathrm{H}_{39} \mathrm{NOSn}$ : C, 62.42; H, 7.86; N, 2.80. Found: C, 62.55; H, 7.58; N, 2.86.

$N$-[2-( $\alpha$-Tributylstannylbenzyl)phenyl]thioformamide (1f): 91\% yield; pale yellow solid; mp 50-51 ${ }^{\circ} \mathrm{C}$; IR (KBr) $v_{\max }$ 2923, 1647, 1551, 1357 (C=S), $1070 \mathrm{~cm}^{-1}$; ${ }^{1} \mathrm{H}-\mathrm{NMR}$ $\left(\mathrm{CDCl}_{3}, 270 \mathrm{MHz}\right) \delta 0.71-0.93(\mathrm{~m}, 15 \mathrm{H}), 1.04-1.36(\mathrm{~m}, 12 \mathrm{H}), 4.02\left(\mathrm{t}, 1 \mathrm{H},{ }^{2} J_{\mathrm{H}-\mathrm{Sn}}=33\right.$ Hz), 6.93-7.01 (m, 3H), 7.11-7.23 (m, 6H), $9.10+9.15$ (2brs, 1H), $9.44+9.49$ (2s, 1H); ${ }^{13} \mathrm{C}-\mathrm{NMR}\left(\mathrm{CDCl}_{3}, 68 \mathrm{MHz}\right) \delta 10.9,13.6,27.2,28.8,36.9,120.7,124.8,126.4,126.5$, 126.6, 128.8, 131.7, 134.3, 137.3, 142.5, 188.8; CI-MS m/z $518\left(\mathrm{MH}^{+}, 66\right), 460\left(\mathrm{M}^{+}-\right.$ Bu, 96), $226\left(\mathrm{M}^{+}-\mathrm{Bu}_{3} \mathrm{Sn}, 32\right), 194\left(\mathrm{M}^{+}-\mathrm{Bu}_{3} \mathrm{Sn}\right.$ - S, 100); Anal. Calcd for $\mathrm{C}_{26} \mathrm{H}_{39} \mathrm{NSSn}$ : C, 60.48; H, 7.61; N, 2.71. Found: C, 60.45; H, 7.42; N, 2.73.

$N$-[2-( $\alpha$-Tributylstannylbenzyl)phenyl]acetamide: 74\% yield; colorless solid; mp 65$66{ }^{\circ} \mathrm{C}$; IR (KBr) $v_{\max } 3274,2927,1653(\mathrm{C}=\mathrm{O}), 1535,1452,1305,744,700 \mathrm{~cm}^{-1} ;{ }^{1} \mathrm{H}-$ NMR (CDCl $3,270 \mathrm{MHz}) \delta$ 0.77-1.06 (m, 15H), 1.16-1.43 (m,12H), 1.92 (s, 3H), 4.03 (t, 
$1 \mathrm{H},{ }^{2} J_{\mathrm{H}-\mathrm{Sn}}=34 \mathrm{~Hz}$ ), 7.01-7.23 (m, 9H), 7.84 (brd, $\left.1 \mathrm{H}, J=7.3 \mathrm{~Hz}\right) ;{ }^{13} \mathrm{C}-\mathrm{NMR}\left(\mathrm{CDCl}_{3}\right.$, $68 \mathrm{MHz}) \delta 10.6,13.6,24.2,27.3,28.9,37.7,123.9,124.4,124.5,126.2,126.4,128.6$, 131.0, 133.9, 135.9, 143.7, 167.8; CI-MS m/z 515 ( $\left.\mathrm{M}^{+}, 25\right), 458\left(\mathrm{M}^{+}-\mathrm{Bu}, 56\right), 179\left(\mathrm{M}^{+}\right.$

- $\mathrm{Bu}_{3} \mathrm{Sn}$ - $\left.\mathrm{CH}_{3} \mathrm{CO}, 100\right)$; Anal. Calcd for $\mathrm{C}_{27} \mathrm{H}_{41} \mathrm{NOSn}$ : C, 63.05; H, 8.03; N, 2.72. Found: C, 63.05; H, 7.93; N, 2.72.

$N$-[2-( $\alpha$-Tributylstannylbenzyl)phenyl]thioacetamide (1g): 86\% yield; pale yellow solid; mp 45-47 ${ }^{\circ} \mathrm{C}$; IR (KBr) $v_{\max }$ 3142, 2939, 1595, 1527, 1491, 1448, 1377 (C=S), 1165, 754, $696 \mathrm{~cm}^{-1} ;{ }^{1} \mathrm{H}-\mathrm{NMR}\left(\mathrm{CDCl}_{3}, 270 \mathrm{MHz}\right) \delta$ 0.75-0.96 (m, 15H), 1.05-1.46 (m,12H), $2.18(\mathrm{~s}, 1.59 \mathrm{H}), 2.59(\mathrm{~s}, 1.41 \mathrm{H}), 4.03\left(\mathrm{t}, 0.47 \mathrm{H},{ }^{2} J_{\mathrm{H}-\mathrm{Sn}}=32 \mathrm{~Hz}\right), 4.05\left(\mathrm{t}, 0.53 \mathrm{H},{ }^{2} J_{\mathrm{H}-\mathrm{Sn}}=\right.$ $31 \mathrm{~Hz}$ ), 7.00-7.44 (m, 8.47H), 7.59 (d, 0.53H, $J=7.3 \mathrm{~Hz}$ ), 8.22 (brs, 0.47H), 8.96 (brs, 0.53H); ${ }^{13} \mathrm{C}-\mathrm{NMR}\left(\mathrm{CDCl}_{3}, 68 \mathrm{MHz}\right) \delta 10.7,13.66,13.71,27.33,27.37,28.89,28.96$, 29.8, 35.0, 36.9, 37.4, 124.3, 124.5, 126.1, 126.2, 126.3, 126.5, 127.5, 127.6, 127.8, 128.47, 128.55, 128.61, 131.8, 132.0, 136.3, 137.4, 138.9, 140.4, 142.9, 144.1, 200.7, 205.1; CI-MS m/z $532\left(\mathrm{MH}^{+}, 16\right), 474\left(\mathrm{M}^{+}-\mathrm{Bu}, 30\right), 240\left(\mathrm{M}^{+}-\mathrm{Bu}_{3} \mathrm{Sn}, 66\right), 208\left(\mathrm{M}^{+}-\right.$ $\mathrm{Bu}_{3} \mathrm{Sn}-\mathrm{S}, 100$ ); Anal. Calcd for $\mathrm{C}_{27} \mathrm{H}_{41} \mathrm{NSSn}$ : C, 61.14; H, 7.79; N, 2.64. Found: C, 60.91; H, 7.61; N, 2.62.

$N$-[2-( $\alpha$-Tributylstannylmethyl)phenyl]benzamide: $5 \%$ yield; colorless solid; mp 43$44{ }^{\circ} \mathrm{C}$; IR (KBr) $v_{\max }$ 2919, 2360, $1647(\mathrm{C}=\mathrm{O}), 1521,1270 \mathrm{~cm}^{-1} ;{ }^{1} \mathrm{H}-\mathrm{NMR}\left(\mathrm{CDCl}_{3}, 270\right.$ MHz) $\delta$ 0.68-0.90 (m, 15H), 1.02-1.47 (m, 12H), $2.26\left(\mathrm{t}, 2 \mathrm{H},{ }^{2} J_{\mathrm{H}-\mathrm{Sn}}=26 \mathrm{~Hz}\right), 7.00-7.09$ (m, 3H), 7.44-7.57 (m, 4H), 7.86-7.89 (m, 3H); ${ }^{13} \mathrm{C}-\mathrm{NMR}\left(\mathrm{CDCl}_{3}, 68 \mathrm{MHz}\right) \delta$ 9.9, 13.7, 14.2, 27.3, 29.0, 123.3, 124.0, 125.2, 126.8, 128.2, 128.6, 131.6, 133.1, 134.9, 135.1, 165.1; CI-MS m/z 502 ( $\left.\mathrm{MH}^{+}, 100\right), 444\left(\mathrm{M}^{+}-\mathrm{Bu}, 48\right), 105$ (PhCO $\left.{ }^{+}, 3\right)$; Anal. Calcd for $\mathrm{C}_{26} \mathrm{H}_{39} \mathrm{NOSn}: \mathrm{C}, 62.42 ; \mathrm{H}, 7.86 ; \mathrm{N}, 2.80$. Found: C, 62.70; H, 7.69; N, 2.83. 
$N$-[2-( $\alpha$-Tributylstannylmethyl)phenyl]thiobenzamide (1h): 77\% yield; yellow oil; IR (neat) $v_{\max }$ 2923, 1601, 1448, $1351(\mathrm{C}=\mathrm{S}) \mathrm{cm}^{-1} ;{ }^{1} \mathrm{H}-\mathrm{NMR}\left(\mathrm{CDCl}_{3}, 270 \mathrm{MHz}\right) \delta 0.67-$ $0.93(\mathrm{~m}, 15 \mathrm{H}), 1.13-1.45(\mathrm{~m}, 12 \mathrm{H}), 2.27\left(\mathrm{t}, 2 \mathrm{H},{ }^{2} J_{\mathrm{H}-\mathrm{Sn}}=26 \mathrm{~Hz}\right), 7.06-7.21(\mathrm{~m}, 3 \mathrm{H})$, 7.40-7.53 (m, 3H), 7.64 (d, 1H, $J=7.6 \mathrm{~Hz}$ ), 7.88 (d, 2H, $J=7.3 \mathrm{~Hz}$ ), 8.66 (brs, 1H); ${ }^{13} \mathrm{C}-\mathrm{NMR}\left(\mathrm{CDCl}_{3}, 68 \mathrm{MHz}\right) \delta 10.0,13.7,14.3,27.3,29.0,123.7,126.4,126.5,127.7$, 128.5, 128.8, 131.1, 134.8, 139.3, 142.3, 198.4; CI-MS m/z $518\left(\mathrm{MH}^{+}, 75\right), 460\left(\mathrm{M}^{+}\right.$Bu, 52), $226\left(\mathrm{M}^{+}-\mathrm{Bu}_{3} \mathrm{Sn}, 100\right), 194\left(\mathrm{M}^{+}-\mathrm{Bu}_{3} \mathrm{Sn}-\mathrm{S}, 37\right)$; Anal. Calcd for $\mathrm{C}_{26} \mathrm{H}_{39} \mathrm{NSSn}$ : C, 60.48; H, 7.61; N, 2.71. Found: C, 60.64; H, 7.41; N, 2.66.

General procedure of thermal reaction of thioanilide $\mathbf{1}$

A benzene solution $(1.5 \mathrm{~mL})$ of thioanilide $1(0.15 \mathrm{mmol})$ in a sealed tube was heated at reaction temperature. After the reaction, the solution was concentrated, and the residue was purified by silica gel column chromatography (eluent: hexane/ethyl acetate) to give the corresponding indole derivative 2 .

Indoles 2a,c,e-h was confirmed by comparison of the spectral data with those reported in the reference. ${ }^{2}$

2-(4'-Methoxyphenyl)-3-phenylindole (2b): 84\% yield; yellow solid; IR (KBr) $v_{\max }$ 3406, 1603, 1510, 1489, 1456, 1250, 1176, 1030, 833, 746, $700 \mathrm{~cm}^{-1} ;{ }^{1} \mathrm{H}-\mathrm{NMR}\left(\mathrm{CDCl}_{3}\right.$, $270 \mathrm{MHz}) \delta 3.79(\mathrm{~s}, 3 \mathrm{H}), 6.81-6.86(\mathrm{~m}, 2 \mathrm{H}), 7.10-7.45$ (m, 10H), 7.66 (d, 1H, $J=7.8$ Hz), 8.11 (brs, $1 \mathrm{H}) ;{ }^{13} \mathrm{C}-\mathrm{NMR}\left(\mathrm{CDCl}_{3}, 68 \mathrm{MHz}\right) \delta$ 55.3, 110.7, 114.0, 119.3, 120.2, 122.3, 125.0, 126.0, 128.4, 128.6, 129.3, 130.0, 134.0, 135.1, 135.6, 159.0; EI-MS m/z $299\left(\mathrm{M}^{+}, 100\right), 284\left(\mathrm{M}^{+}-\mathrm{Me}, 19\right)$. 
3-(4'-Chlorophenyl)-2-phenylindole (2d): 82\% yield; colorless needle; mp 152-154 ${ }^{\circ} \mathrm{C}$; IR (KBr) $v_{\max } 3398,1500,1450,1325,1248,1093,1014,968,841,746 \mathrm{~cm}^{-1} ;{ }^{1} \mathrm{H}-\mathrm{NMR}$ $\left(\mathrm{CDCl}_{3}, 270 \mathrm{MHz}\right) \delta$ 7.13-7.44 (m, 12H), 7.63 (d, 1H, $\left.J=7.8 \mathrm{~Hz}\right), 8.23$ (brs, $\left.1 \mathrm{H}\right) ;{ }^{13} \mathrm{C}-$ NMR $\left(\mathrm{CDCl}_{3}, 68 \mathrm{MHz}\right) \delta 110.9,113.7,119.3,120.5,122.8,127.8,128.1,128.3,128.6$, 128.7, 131.2, 131.9, 132.2, 133.5, 134.2, 135.7; EI-MS m/z $303\left(\mathrm{M}^{+}, 100\right), 267\left(\mathrm{M}^{+}-\right.$ HCl, 38); Anal. Calcd for $\mathrm{C}_{20} \mathrm{H}_{14} \mathrm{ClN}$ : C, 79.07; H, 4.65; N, 4.61. Found: C, 78.90; H, $4.61 ; \mathrm{N}, 4.60$.

General procedure of preparation of $o$-tributylstannylmethylated aryl isothiocyanate derivatives $^{3}$

A chloroform $(6 \mathrm{~mL})$ solution of an $o$-tributylstannylmethylated aniline derivative (5 mmol) was added to a suspension of thiophosgen $(7.5 \mathrm{mmol})$ and calcium carbonate $(0.75 \mathrm{~g}, 7.5 \mathrm{mmol})$ in chloroform $(6 \mathrm{~mL})$ and water $(2 \mathrm{~mL})$ at $0{ }^{\circ} \mathrm{C}$ and stirred for $30 \mathrm{~min}$. After the reaction, to the mixture was added a saturated sodium chloride aqueous solution. The organic layer was separated from the mixture, and the aqueous layer was extracted with chloroform $(5 \mathrm{~mL})$. The combined organic layers were combined, and dried over anhydrous magnesium sulfate. The solution was concentrated, and the residue was purified by silica gel column chromatography (eluent: hexane / ethyl acetate) to give the corresponding o-tributylstannylmethylated phenyl isocyanate derivative 3 .

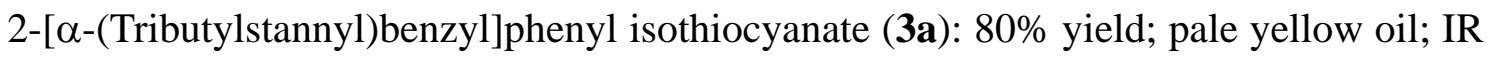
(neat) $v_{\max }$ 2925, $2089(\mathrm{~N}=\mathrm{C}=\mathrm{S}), 1591,1448,752 \mathrm{~cm}^{-1} ;{ }^{1} \mathrm{H}-\mathrm{NMR}\left(\mathrm{CDCl}_{3}, 270 \mathrm{MHz}\right) \delta$ 0.75-0.96 (m, 15H), 1.04-1.45 (m, 12H), $4.33\left(\mathrm{t}, 1 \mathrm{H},{ }^{2} J_{\mathrm{H}-\mathrm{Sn}}=32 \mathrm{~Hz}\right.$ ), 7.00-7.30 (m, 
$9 \mathrm{H}) ;{ }^{13} \mathrm{C}-\mathrm{NMR}\left(\mathrm{CDCl}_{3}, 68 \mathrm{MHz}\right) \delta 11.0,13.7,27.3,28.9,38.0,124.6,125.3,127.1$, 127.3, 127.9, 128.3, 128.6, 129.1, 136.7, 140.7, 142.8; CI-MS m/z $516\left(\mathrm{MH}^{+}, 78\right), 458$ ( $\mathrm{M}^{+}$- Bu, 100), $291\left(\mathrm{Bu}_{3} \mathrm{Sn}^{+}, 27\right), 224\left(\mathrm{M}^{+}-\mathrm{Bu}_{3} \mathrm{Sn}, 10\right)$; Anal. Calcd for $\mathrm{C}_{26} \mathrm{H}_{37} \mathrm{NSSn}$ : C, 60.71; H, 7.25; N, 2.72. Found: C, 60.57; H, 7.26; N, 2.80.

4-Chloro-2-[ $\alpha$-(tributylstannyl)benzyl]phenyl isothiocyanate (3b): 77\% yield; pale yellow oil; IR (neat) $v_{\max }$ 2924, $2079(\mathrm{~N}=\mathrm{C}=\mathrm{S}), 1470,939 \mathrm{~cm}^{-1} ;{ }^{1} \mathrm{H}-\mathrm{NMR}\left(\mathrm{CDCl}_{3}, 270\right.$ MHz) $\delta$ 0.75-1.00 (m, 15H), 1.15-1.43 (m, 12H), 4.26 (t, 1H, $\left.{ }^{2} J_{\mathrm{H}-\mathrm{Sn}}=32 \mathrm{~Hz},\right), 6.99-7.05$ (m, 1H), 7.10-7.18 (m, 4H), 7.24-7.31 (m, 3H); ${ }^{13} \mathrm{C}-\mathrm{NMR}\left(\mathrm{CDCl}_{3}, 68 \mathrm{MHz}\right) \delta 11.2$, 13.7, 27.4, 28.9, 38.3, 125.1, 125.3, 126.9, 128.27, 128.37, 128.39, 128.6, 132.8, 141.9, 142.8; EI-MS m/z $549\left(\mathrm{M}^{+}, 1\right), 492\left(\mathrm{M}^{+}-\mathrm{Bu}, 100\right), 291\left(\mathrm{Bu}_{3} \mathrm{Sn}^{+}, 22\right), 258\left(\mathrm{M}^{+}-\mathrm{Bu}_{3} \mathrm{Sn}\right.$, 23); Anal. Calcd for $\mathrm{C}_{26} \mathrm{H}_{36}$ ClNSSn: C, 56.90; H, 6.61; N, 2.55. Found: C, $56.80 \mathrm{H}$, $6.76 ; \mathrm{N}, 2.59$.

4-Methoxy-2-[ $\alpha$-(tributylstannyl)benzyl]phenyl isothiocyanate (3c): 71\% yield; pale yellow oil; IR (neat) $v_{\max }$ 2926, $2096(\mathrm{~N}=\mathrm{C}=\mathrm{S}), 1598,1488 \mathrm{~cm}^{-1} ;{ }^{1} \mathrm{H}-\mathrm{NMR}\left(\mathrm{CDCl}_{3}, 270\right.$ MHz) $\delta$ 0.80-1.00 (m, 15H), 1.15-1.42 (m, 12H), 3.75 (s, 3H), $4.26\left(\mathrm{t}, 1 \mathrm{H},{ }^{2} J_{\mathrm{H}-\mathrm{Sn}}=32\right.$ $\mathrm{Hz}$,), 6.59 (dd, 1H, $J=3.0,8.6 \mathrm{~Hz}), 6.82(\mathrm{~d}, 1 \mathrm{H}, J=3.0 \mathrm{~Hz}), 7.07-7.29(\mathrm{~m}, 6 \mathrm{H}) ;{ }^{13} \mathrm{C}-$ NMR $\left(\mathrm{CDCl}_{3}, 68 \mathrm{MHz}\right) \delta 11.0,13.7,27.4,28.9,38.2,55.4,110.6,114.3,121.1,124.8$, 128.1, 128.26, 128.32, 128.4, 142.4, 142.5, 158.3; CI-MS m/z $546\left(\mathrm{MH}^{+}, 100\right), 488\left(\mathrm{M}^{+}\right.$ - Bu, 70), $291\left(\mathrm{Bu}_{3} \mathrm{Sn}^{+}, 38\right), 256\left(\mathrm{M}^{+}-\mathrm{Bu}_{3} \mathrm{Sn}, 35\right)$; Anal. Calcd for $\mathrm{C}_{27} \mathrm{H}_{39} \mathrm{NOSSn}$ : C, 59.57; H, 7.22; N, 2.57. Found: C, 59.39; H, 7.01; N, 2.57.

2-[4-Chlorophenyl(tributylstannyl)methyl]phenyl isothiocyanate (3d): 83\% yield; pale 
yellow oil; IR (neat) $v_{\max } 2085(\mathrm{~N}=\mathrm{C}=\mathrm{S}) \mathrm{cm}^{-1} ;{ }^{1} \mathrm{H}-\mathrm{NMR}\left(\mathrm{CDCl}_{3}, 270 \mathrm{MHz}\right) \delta 0.75-1.02$ (m, 15H), $1.15-1.41(\mathrm{~m}, 12 \mathrm{H}), 4.26\left(\mathrm{t}, 1 \mathrm{H},{ }^{2} J_{\mathrm{H}-\mathrm{Sn}}=32 \mathrm{~Hz},\right), 7.04-7.26(\mathrm{~m}, 8 \mathrm{H}) ;{ }^{13} \mathrm{C}-$ NMR (CDCl 3 , $68 \mathrm{MHz}) \delta 11.0,13.7,27.4,28.9,37.3,125.7,127.2,127.5,128.4,128.8$, 128.9, 129.2, 130.1, 140.1, 141.6; CI-MS m/z $550\left(\mathrm{MH}^{+}\right.$, 65), 492 (M+ $\left.\mathrm{M}^{+} \mathrm{Bu}, 63\right), 291$ $\left(\mathrm{Bu}_{3} \mathrm{Sn}^{+}, 100\right)$; Anal. Calcd for $\mathrm{C}_{26} \mathrm{H}_{36} \mathrm{ClNSSn}$ : C, 56.90; H, 6.61; N, 2.55. Found: C, 57.17 H, 6.47; N, 2.59.

General procedure of thermal reaction of o-tributylstannylmethylated aryl isothiocyanate derivative 3

A benzene solution (3 mL) of $o$-tributylstannylmethylated aryl isothiocyanate derivative $3(0.3 \mathrm{mmol})$ in a sealed tube was heated at reaction temperature. After the reaction, the solution was concentrated, and the residue was purified by silica gel column chromatography (the silica gel dryied at $150{ }^{\circ} \mathrm{C}$ under reduced pressure, eluent: hexane / ethyl acetate) under nitrogen atmosphere to give the corresponding 2-indolyl sulufide 4. When a undried silica gel was employed in the purification of the mixture under air, disulfide 5 was obtained.

2-Tributylstannylthio-3-phenylindole (4a): 91\% yield; yellow solid; mp 30-32 ${ }^{\circ} \mathrm{C}$; IR (KBr) $v_{\max }$ 3404, 2937, 1521, 1439, 1406, 1346, 1244, 873, 770, $743 \mathrm{~cm}^{-1}$; ${ }^{1} \mathrm{H}-\mathrm{NMR}$ $\left(\mathrm{CDCl}_{3}, 270 \mathrm{MHz}\right) \delta$ 0.74-0.97 (m, 15H), 1.07-1.21 (m, 6H), 1.26-1.46 (m, 6H), 7.057.18 (m, 2H), 7.25-7.31 (m, 2H), 7.41-7.46 (m, 2H), 7.63-7.66 (m, 1H), 7.72-7.75 (m, 2H), 7.99 (brs, $1 \mathrm{H}) ;{ }^{13} \mathrm{C}-\mathrm{NMR}\left(\mathrm{CDCl}_{3}, 68 \mathrm{MHz}\right) \delta$ 13.6, 14.9, 27.0, 28.4, 109.8, 118.6, 119.9, 120.5, 121.9, 125.4, 125.9, 127.8, 128.0, 129.8, 135.0, 135.6; CI-MS m/z 516 ( $\left.\mathrm{MH}^{+}, 100\right), 291\left(\mathrm{Bu}_{3} \mathrm{Sn}^{+}, 52\right)$; Anal. Calcd for $\mathrm{C}_{26} \mathrm{H}_{37} \mathrm{NSSn}$ : C, 60.71; H, 7.25; N, 2.72. 
Found: C, 60.87; H, 6.97; N, 2.82.

Bis(3-phenyl-2-indolyl) disulfide (5a): 88\% yield; yellow soild; mp 196-198 ${ }^{\circ} \mathrm{C}$ (lit. ${ }^{4}$ 196-197.5 $\left.{ }^{\circ} \mathrm{C}\right)$; IR (KBr) $v_{\max } 3388,1521,1479,1439,1402,1352,1329,1244,1151$, 1012, 970, 829, 773, 744, 698, $671 \mathrm{~cm}^{-1}$; ${ }^{1} \mathrm{H}-\mathrm{NMR}\left(\mathrm{CDCl}_{3}, 270 \mathrm{MHz}\right) \delta$ 7.09-7.30 (m, 16H), 7.59 (d, $2 \mathrm{H}, J=8.1 \mathrm{~Hz}$ ), 8.08 (brs, $2 \mathrm{H}) ;{ }^{13} \mathrm{C}-\mathrm{NMR}\left(\mathrm{CDCl}_{3}, 68 \mathrm{MHz}\right) \delta 111.0$, 120.2, 120.6, 124.3, 124.5, 125.0, 126.8, 127.0, 128.1, 129.8, 133.1, 137.0; EI-MS m/z $448\left(\mathrm{M}^{+}, 5\right), 416\left(\mathrm{M}^{+}-\mathrm{S}, 15\right), 224\left(\mathrm{M}^{+} / 2,96\right), 223\left(\mathrm{M}^{+} / 2-\mathrm{H}, 100\right)$; Anal. Calcd for $\mathrm{C}_{28} \mathrm{H}_{20} \mathrm{~N}_{2} \mathrm{~S}_{2}$ : C, 74.97; H, 4.49; N, 6.24. Found: C, 74.72; H, 4.59; N, 6.10.

Bis(5-chloro-3-phenyl-2-indolyl) disulfide (5b): 96\% yield; yellow solid; mp 193$195{ }^{\circ} \mathrm{C}$; IR (KBr) $v_{\max } 3385,1601,1525,1483,1433,1402,1348,1267,1240,1198$, 1066, 939, 800, 771, 700, 673, $592 \mathrm{~cm}^{-1}$; ${ }^{1} \mathrm{H}-\mathrm{NMR}\left(\mathrm{CDCl}_{3}, 270 \mathrm{MHz}\right) \delta$ 7.09-7.25 (m, 14H), 7.53-7.54 (m, 2H), 8.07 (brs, 2H); ${ }^{13} \mathrm{C}-\mathrm{NMR}\left(\mathrm{CDCl}_{3}, 68 \mathrm{MHz}\right) \delta 112.1,119.5$, 124.6, 124.7, 125.6, 126.4, 127.0, 127.8, 128.3, 129.6, 132.3, 135.1; EI-MS m/z 516 $\left(\mathrm{M}^{+}, 11\right), 259\left(\mathrm{M}^{+} / 2+\mathrm{H}, 62\right), 223\left(\mathrm{M}^{+} / 2+\mathrm{H}-\mathrm{S}, 100\right)$; Anal. Calcd for $\mathrm{C}_{28} \mathrm{H}_{18} \mathrm{Cl}_{2} \mathrm{~N}_{2} \mathrm{~S}_{2}$ : C, 64.99; H, 3.51; N, 5.41. Found: C, 64.97; H, 3.54; N, 5.38.

Bis(5-methoxy-3-phenyl-2-indolyl) disulfide (5c): 94\% yield; yellow solid; mp 172$174{ }^{\circ} \mathrm{C}$; IR (KBr) $v_{\max } 3415,3317,2951,1620,1493,1450,1433,1292,1257,1221$, 1134, 1117, 1034, 957, 839, 791, 764, $702 \mathrm{~cm}^{-1}$; ${ }^{1} \mathrm{H}-\mathrm{NMR}\left(\mathrm{CDCl}_{3}, 270 \mathrm{MHz}\right) \delta 3.79$ (s, 6H), 6.93-7.01 (m, 4H), 7.12-7.26 (m, 12H), 8.00 (brs, $2 \mathrm{H}) ;{ }^{13} \mathrm{C}-\mathrm{NMR}\left(\mathrm{CDCl}_{3}, 68\right.$ MHz) $\delta 55.8,100.9,111.9,115.3,124.7,125.0,126.7,127.3,128.2,129.7,132.2,133.3$, 154.7; EI-MS m/z $477\left(\mathrm{M}^{+}\right.$- OMe, 28), $256\left(\mathrm{M}^{+} / 2+2 \mathrm{H}, 100\right)$; Anal. Calcd for 
$\mathrm{C}_{30} \mathrm{H}_{24} \mathrm{~N}_{2} \mathrm{O}_{2} \mathrm{~S}_{2}$ : C, 70.84; H, 4.76; N, 5.51. Found: C, 70.72; H, 4.76; N, 5.45.

Bis[3-(4-chlorophenyl)-2-indolyl] disulfide (5d): 85\% yield; yellow solid; mp 201$204{ }^{\circ} \mathrm{C}$; IR (KBr) $v_{\max } 3383,1522,1477,1329,1149,1090,1014,839,743 \mathrm{~cm}^{-1} ;{ }^{1} \mathrm{H}-$ NMR $\left(\mathrm{CDCl}_{3}, 270 \mathrm{MHz}\right) \delta$ 6.97-7.35 (m, 14H), 7.50 (d, 2H, $\left.J=8.1 \mathrm{~Hz}\right), 8.10$ (brs, 2H);

${ }^{13} \mathrm{C}-\mathrm{NMR}\left(\mathrm{CDCl}_{3}, 68 \mathrm{MHz}\right) \delta 111.1,119.9,120.9,123.8,124.89,124.91,126.5,128.1$, 130.7, 131.2, 132.5, 137.0; CI-MS m/z $517\left(\mathrm{MH}^{+}, 1\right), 485\left(\mathrm{MH}^{+}-\mathrm{S}, 41\right), 260\left(\mathrm{M}^{+} / 2+\right.$ $2 \mathrm{H}, 100$ ); Anal. Calcd for $\mathrm{C}_{28} \mathrm{H}_{18} \mathrm{Cl}_{2} \mathrm{~N}_{2} \mathrm{~S}_{2}$ : C, 64.99; H, 3.51; N, 5.41. Found: C, 65.10; H, 3.60; N, 5.29.

Procedure of one-pot reaction of phenyl isothiocyanate derivative 3a to 2-methylthio-3phenylindole (6)

A benzene solution (3 mL) of phenyl isothiocyanate derivative 3a (154 mg, 0.3 mmol) was heated at $150{ }^{\circ} \mathrm{C}$ for $15 \mathrm{~h}$, and was concentrated. Acetonitrile solution (3 $\mathrm{mL}$ ) of methyl iodide $(0.0187 \mathrm{~mL})$ and tetrabutylammonium fluoride (1.0 M THF solution, $0.3 \mathrm{~mL}$ ) were added to the residue. The mixture was stirred for $30 \mathrm{~min}$ at room temperature, and concentrated. Diethyl ether $(20 \mathrm{~mL})$ were added to the residue, and the mixture was washed with a saturated potassium fluoride aqueous solution (20 $\mathrm{mL})$ and a saturated sodium chloride aqueous solution $(20 \mathrm{~mL})$. The organic layer was dried over anhydrous magnesium sulfate and concentrated. The mixture was purified by silica gel column chromatography (eluent: hexane/ethyl acetate) to give 2methylthio-3-phenylindole (6, 88\% yield).

2-Methylthio-3-phenylindole (6): colorless plate; mp 84-86 ${ }^{\circ} \mathrm{C}$ (lit. $\left.{ }^{5} 84-86{ }^{\circ} \mathrm{C}\right)$; IR (KBr) $v_{\max } 3477,1603,1529,1485,1439,1408,1352,1327,1244,1038,970,775,754,704$, 
$679 \mathrm{~cm}^{-1} ;{ }^{1} \mathrm{H}-\mathrm{NMR}\left(\mathrm{CDCl}_{3}, 270 \mathrm{MHz}\right) \delta 2.30$ (s, 3H), 7.09-7.19 (m, 1H), 7.22-7.25 (m, 1H), 7.29-7.36 (m, 2H), 7.43-7.49 (m, 2H), 7.63-7.70 (m, 3H), 8.11 (brs, $1 \mathrm{H})$; ${ }^{13} \mathrm{C}-\mathrm{NMR}$ $\left(\mathrm{CDCl}_{3}, 68 \mathrm{MHz}\right) \delta 19.5,110.5,119.4,120.3,121.1,123.0,126.4,126.8,127.4,128.2$, 129.6, 134.1, 136.1; CI-MS m/z 239 ( $\left.\mathrm{M}^{+}, 76\right), 224\left(\mathrm{M}^{+}\right.$- Me, 100); Anal. Calcd for $\mathrm{C}_{15} \mathrm{H}_{13} \mathrm{NS}$ : C, 75.27; H, 5.47; N, 5.85. Found: C, 75.15; H, 5.28; N, 5.95.

Procedure of one-pot reaction of phenyl isothiocyanate derivative 3a to 3-phenyl-2indolyl 2-pyridyl sulfide (7)

A benzene solution (3 mL) of phenyl isothiocyanate derivative 3a (168 mg, $0.33 \mathrm{mmol}$ ) was heated at $150{ }^{\circ} \mathrm{C}$ for $15 \mathrm{~h}$ in a sealed tube and was cooled to room temperature. The sealed tube was opened, and then tetrakis(triphenylphosphine)palladium (38.1 mg, $0.33 \mathrm{mmol})$ and 2-bromopyridine $(0.0314 \mathrm{~mL}, 0.33 \mathrm{mmol})$ were added to the solution. The solution was heated for $24 \mathrm{~h}$ at $100{ }^{\circ} \mathrm{C}$ in a sealed tube and concentrated. Diethyl ether (20 mL) were added to the residue, and the mixture was washed with a saturated potassium fluoride aqueous solution $(20 \mathrm{~mL})$ and a saturated sodium chloride aqueous solution $(20 \mathrm{~mL})$. The organic layer was dried over anhydrous magnesium sulfate and concentrated. The mixture was purified by silica gel column chromatography (eluent: hexane / ethyl acetate) to give S-3-phenyl-2-indolyl 2-pyridyl sulfide (7, 86\% yield). 3-Phenyl-2-indolyl 2-pyridyl sulfide (7): mp 159-160 ${ }^{\circ} \mathrm{C}$; IR (KBr) $v_{\max } 3199,2918$, 1603, 1576, 1448, 1416, 1356, 1246, 1128, 752, $700 \mathrm{~cm}^{-1} ;{ }^{1} \mathrm{H}-\mathrm{NMR}\left(\mathrm{CDCl}_{3}, 270 \mathrm{MHz}\right)$ $\delta 6.86(\mathrm{dt}, 1 \mathrm{H}, J=0.8,7.3 \mathrm{~Hz}), 6.98$ (ddd, $1 \mathrm{H}, J=1.0,4.9,7.4 \mathrm{~Hz}), 7.14-7.62(\mathrm{~m}, 9 \mathrm{H})$, 7.77 (d, $1 \mathrm{H}, J=8.1 \mathrm{~Hz}$ ), 8.40 (ddd, $1 \mathrm{H}, J=0.8,1.8,4.9 \mathrm{~Hz}$ ), 9.29 (brs, $1 \mathrm{H}$ ); ${ }^{13} \mathrm{C}-\mathrm{NMR}$ $\left(\mathrm{CDCl}_{3}, 68 \mathrm{MHz}\right) \delta 111.1,120.0,120.3,120.4,120.6,121.2,123.6,126.7,127.0,128.3$, 129.5, 133.6, 137.0, 149.3, 159.9; EI-MS m/z $302\left(\mathrm{M}^{+}, 100\right), 269\left(\mathrm{M}^{+}-\mathrm{SH}, 20\right), 223$ 
( $\left.\mathrm{M}^{+}-\mathrm{Py}-\mathrm{H}, 35\right)$; Anal. Calcd for $\mathrm{C}_{19} \mathrm{H}_{14} \mathrm{~N}_{2} \mathrm{~S}$ : C, 75.47; H, 4.67; N, 9.26. Found: C, 75.43; H, 4.76; N, 9.30.

Procedure of one-pot reaction of phenyl isothiocyanate derivative 3a to S-3-phenyl-2indolyl benzothioate (8)

A benzene solution ( $3 \mathrm{~mL}$ ) of phenyl isothiocyanate derivative $3 a$ (154 mg, $0.3 \mathrm{mmol}$ ) was heated at $150{ }^{\circ} \mathrm{C}$ for $15 \mathrm{~h}$, and the solution was concentrated. Chloroform (3 mL) solution of benzoyl chloride $(0.0348 \mathrm{~mL}, 0.3 \mathrm{mmol})$ was added to the residue. The mixture was refluxed for $8 \mathrm{~h}$, and concentrated. Diethyl ether (20 mL) was added to the residue, and the mixture was washed with a saturated potassium fluoride aqueous solution (20 mL) and then a saturated sodium chloride aqueous solution (20 mL). The organic layer was dried over anhydrous magnesium sulfate and concentrated. The mixture was purified by silica gel column chromatography (eluent: hexane / ethyl acetate) to give $S$-3-phenyl-2-indolyl benzothioate (8, 74\% yield).

S-3-phenyl-2-indolyl benzothioate (8): pale yellow needle; mp $153-154{ }^{\circ} \mathrm{C}$; IR (KBr) $v_{\max } 3369,1672,1485,1444,1400,1331,1246,1209,1174,893,773,750,687 \mathrm{~cm}^{-1}$; ${ }^{1} \mathrm{H}-\mathrm{NMR}\left(\mathrm{CDCl}_{3}, 270 \mathrm{MHz}\right) \delta$ 7.14-7.74 (m, 12H), 7.99-8.03 (m, 2H), 9.02 (brs, 1H);

${ }^{13} \mathrm{C}-\mathrm{NMR}\left(\mathrm{CDCl}_{3}, 68 \mathrm{MHz}\right) \delta 111.1,117.7,119.7,120.4,123.5,123.6,126.8,126.9$, 127.5, 128.3, 128.8, 129.7, 133.5, 134.0, 136.0, 137.3, 190.2; EI-MS m/z $329\left(\mathrm{M}^{+}, 23\right)$, $105\left(\mathrm{PhCO}^{+}, 100\right)$.

\section{Time Course of Thermal Reaction of Thioanilide 1a}

$d_{6}$-Benzene solutions of thioanilide 1a (initial concentrations : $2.0 \times 10^{-1}, 1.0 \times 10^{-1}$, and 
$5.0 \times 10^{-2} \mathrm{M}$ ) were heated in sealed NMR tubes, and yields were determined by ${ }^{1} \mathrm{H}$ NMR. Reaction rates of the reactions are the same.

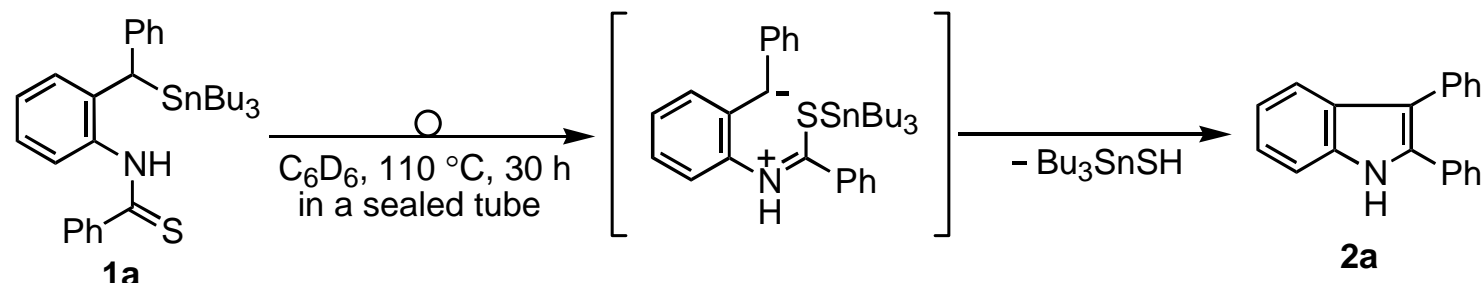

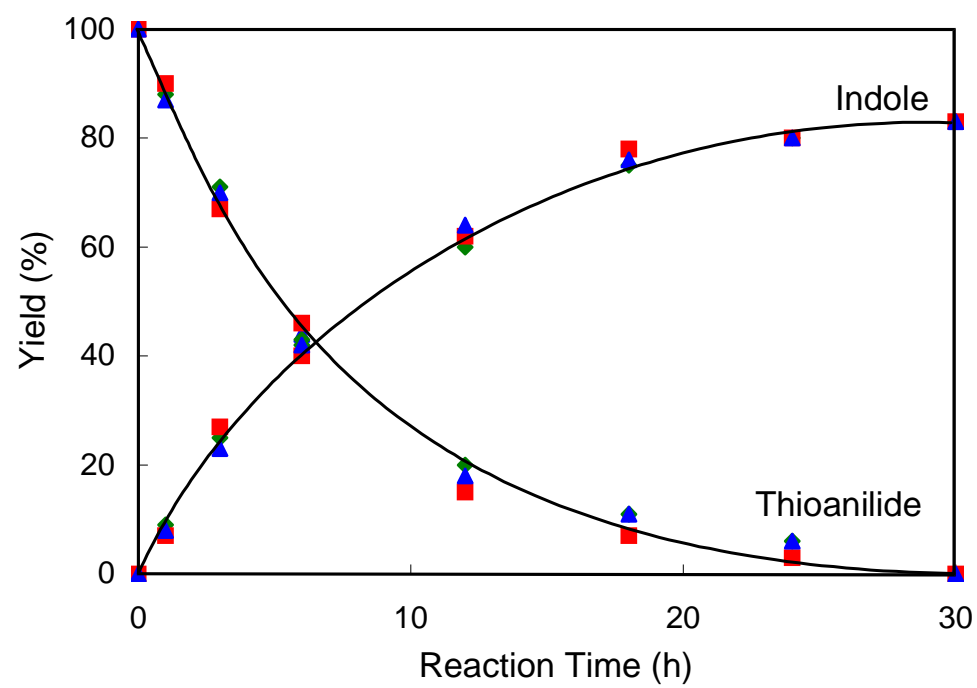

Initial Concentration of Thioanilide

- $2.0 \times 10^{-1} \mathrm{M}$

$1.0 \times 10^{-1} \mathrm{M}$

$\Delta 5.0 \times 10^{-2} \mathrm{M}$

\section{Reference}

(1) A. B. Smith III, R. Mewshaw, J. Org. Chem. 1984, 49, 3685-3689.

(2) A. Fürstner, D. N. Jumbam, Tetrahedron 1992, 48, 5991-6010.

(3) H. Tokuyama, T. Yamashita, M. T. Reding, Y. Kaburagi, T. Fukuyama, J. Am. Chem. Soc. 1999, 121, 3791-3792.

(4) B. D. Palmer, G. W. Rewcastle, A. M. Thompson, M. Boyd, H. D. H. Showalter, A.

D. Sercel, D. W. Fry, A. J. Kraker, W. A. Denny, J. Med. Chem. 1995, 38, 58-67.

(5) D. Lednicer, E. Emmat, J. Heterocyclic Chem. 1971, 8, 903-910. 
M1-OH-P11

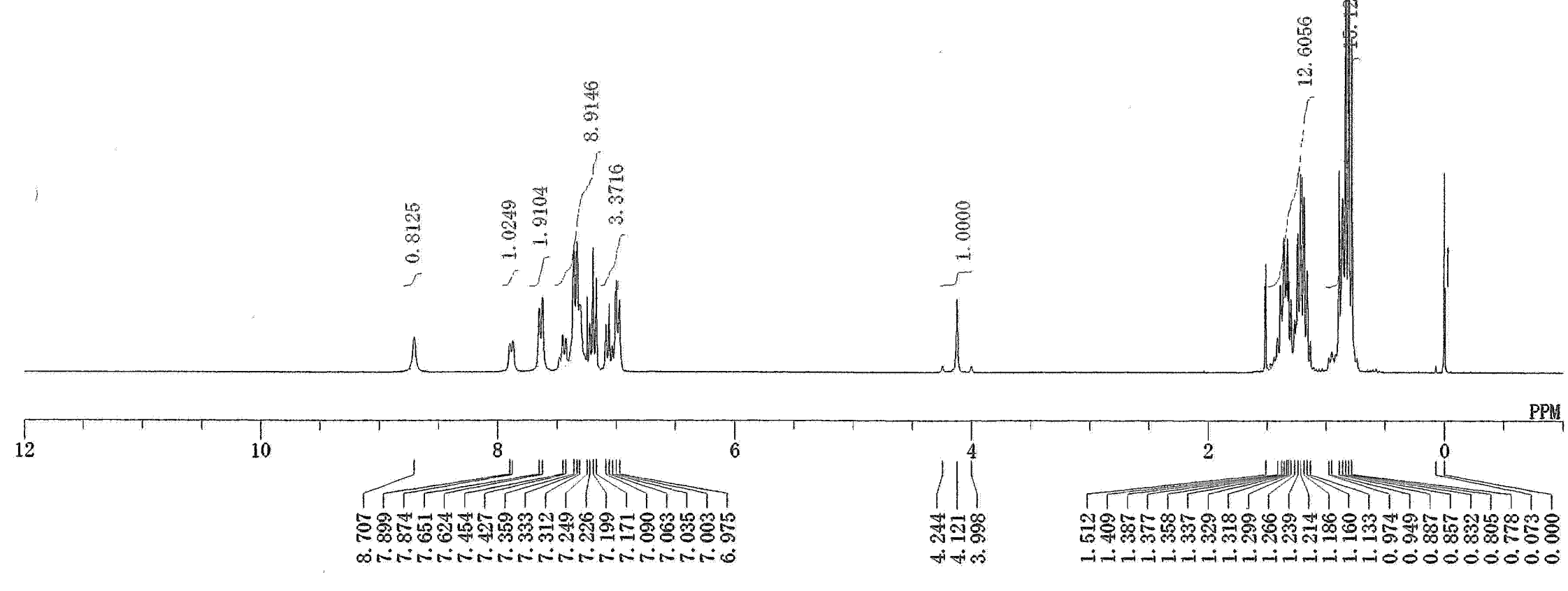




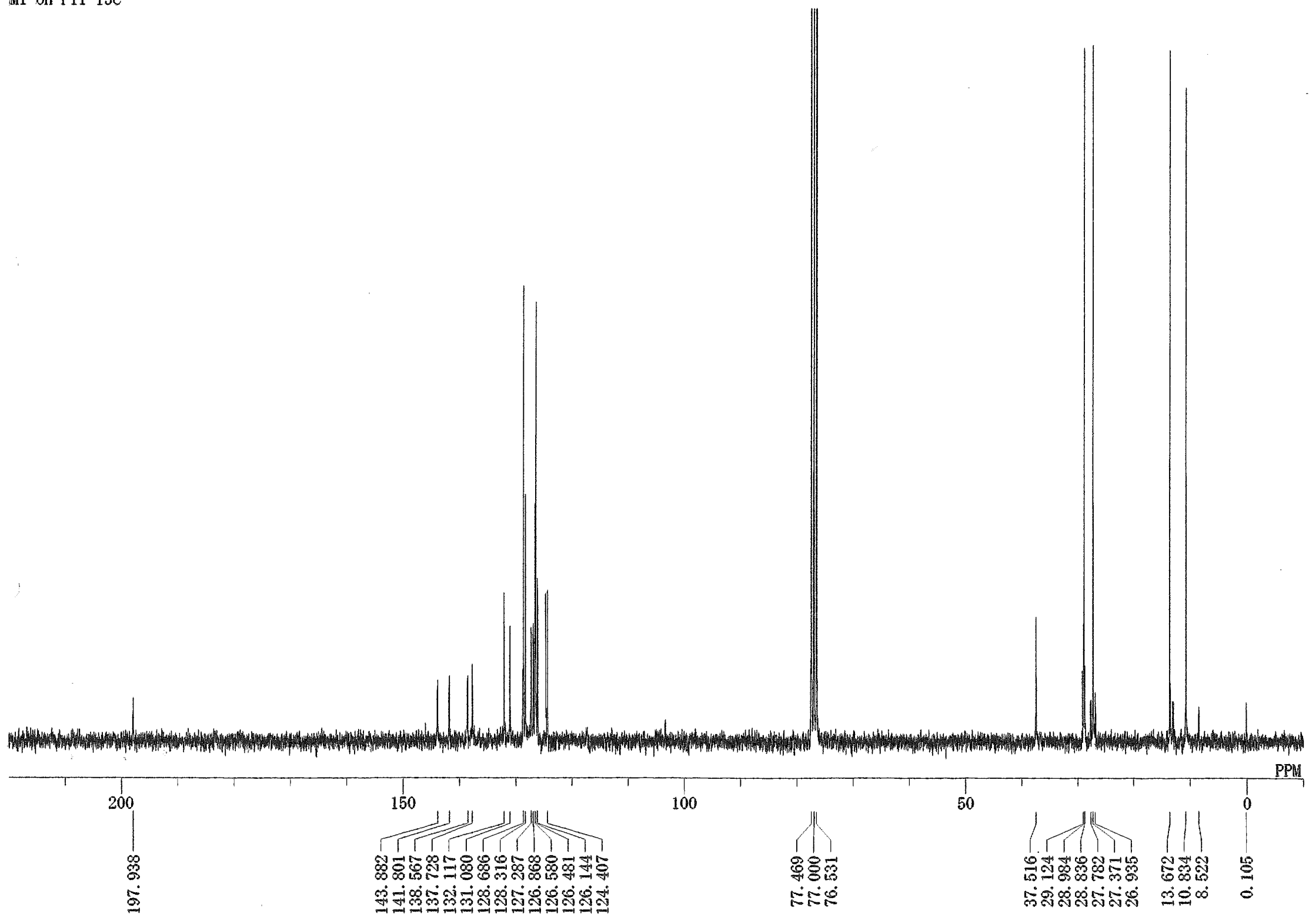


M1-OH-P44

${ }_{O}^{\mathrm{Sh}} \mathrm{O}_{\mathrm{S}}^{\mathrm{H}} \mathrm{OC}^{\mathrm{OHe}}$

$1 b$

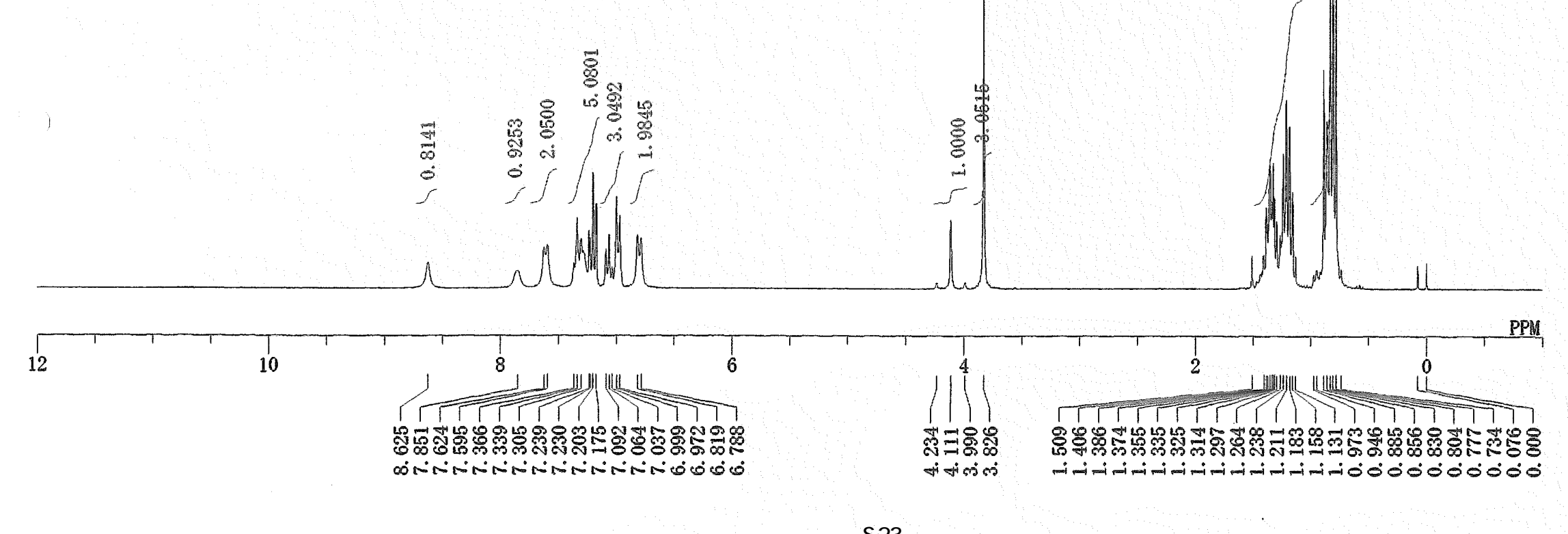


$\backslash$ Komanmr \personal \ota \preparation $\backslash P 44 \backslash P 44-13 \mathrm{C}$.als

$\mathrm{M} 1-\mathrm{OH}-\mathrm{P} 44$

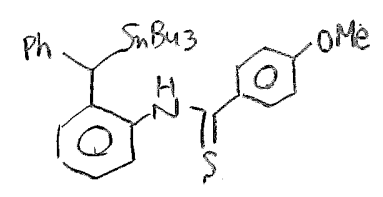

$1 b$

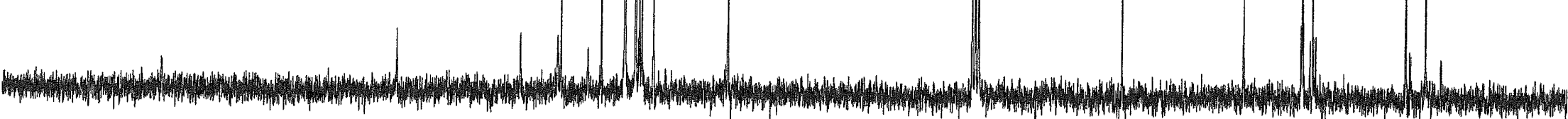

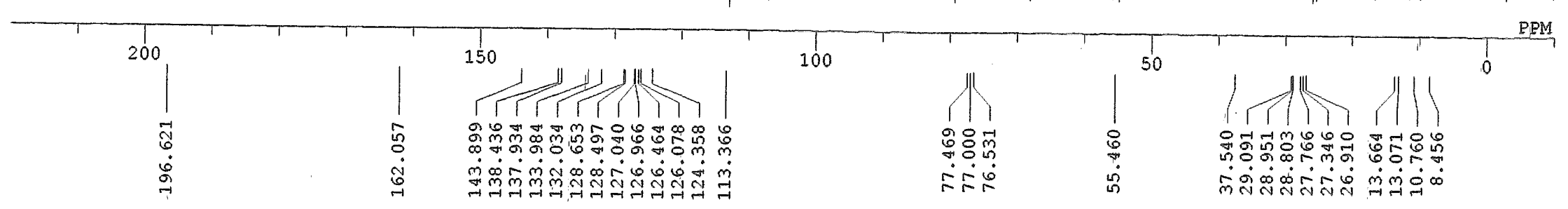


M1-0H-P38

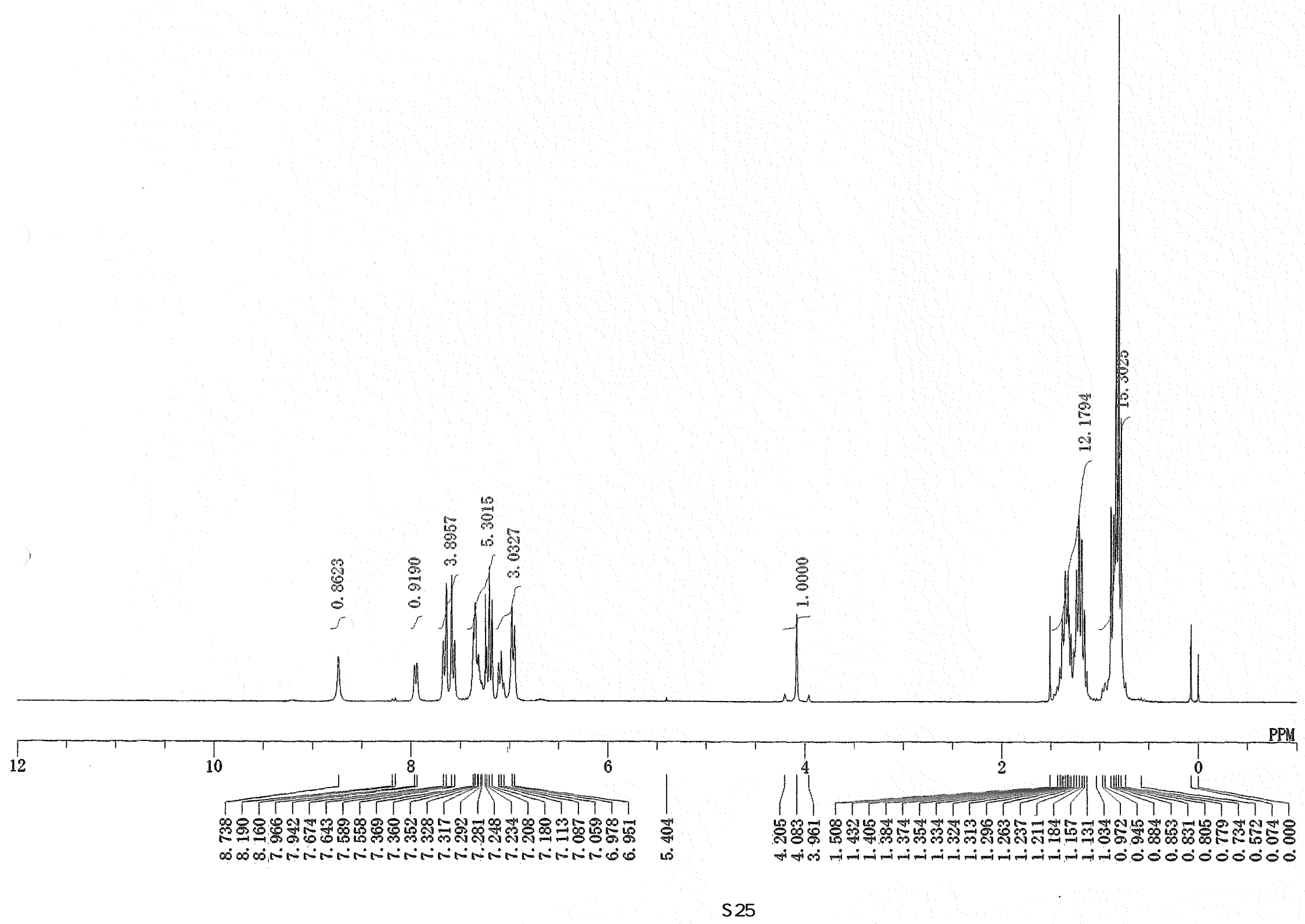

$\int_{0}^{\mathrm{SnBu} 3} \pi^{H} \pi^{\mathrm{CF}}$

$1 c$ 
\KKomanmr \personal \ota \preparation \P38\P38-13C'.als M1-OH-P38-13C

$\mathrm{C}_{10}^{\mathrm{SnBus}} \mathrm{C}_{5}^{\mathrm{CF}}$

$1 \mathrm{c}$

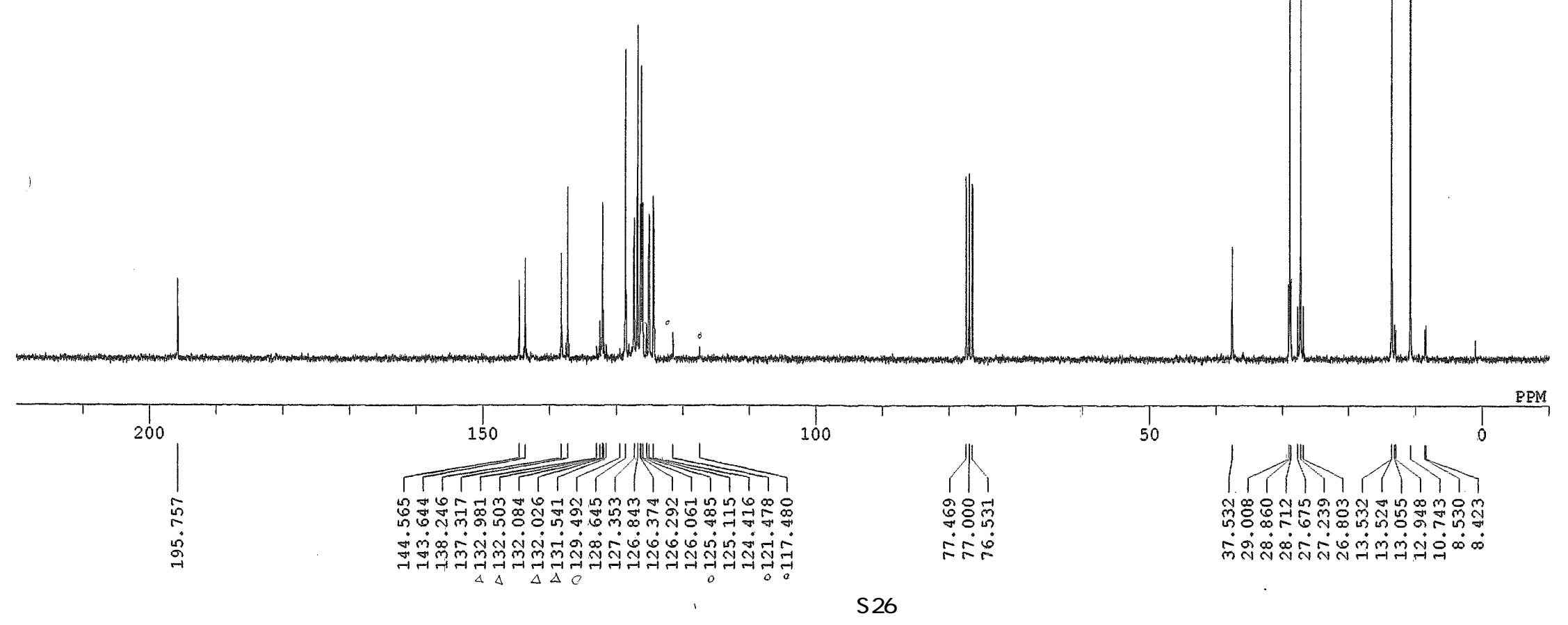




\section{M2-OH-P87-228K}

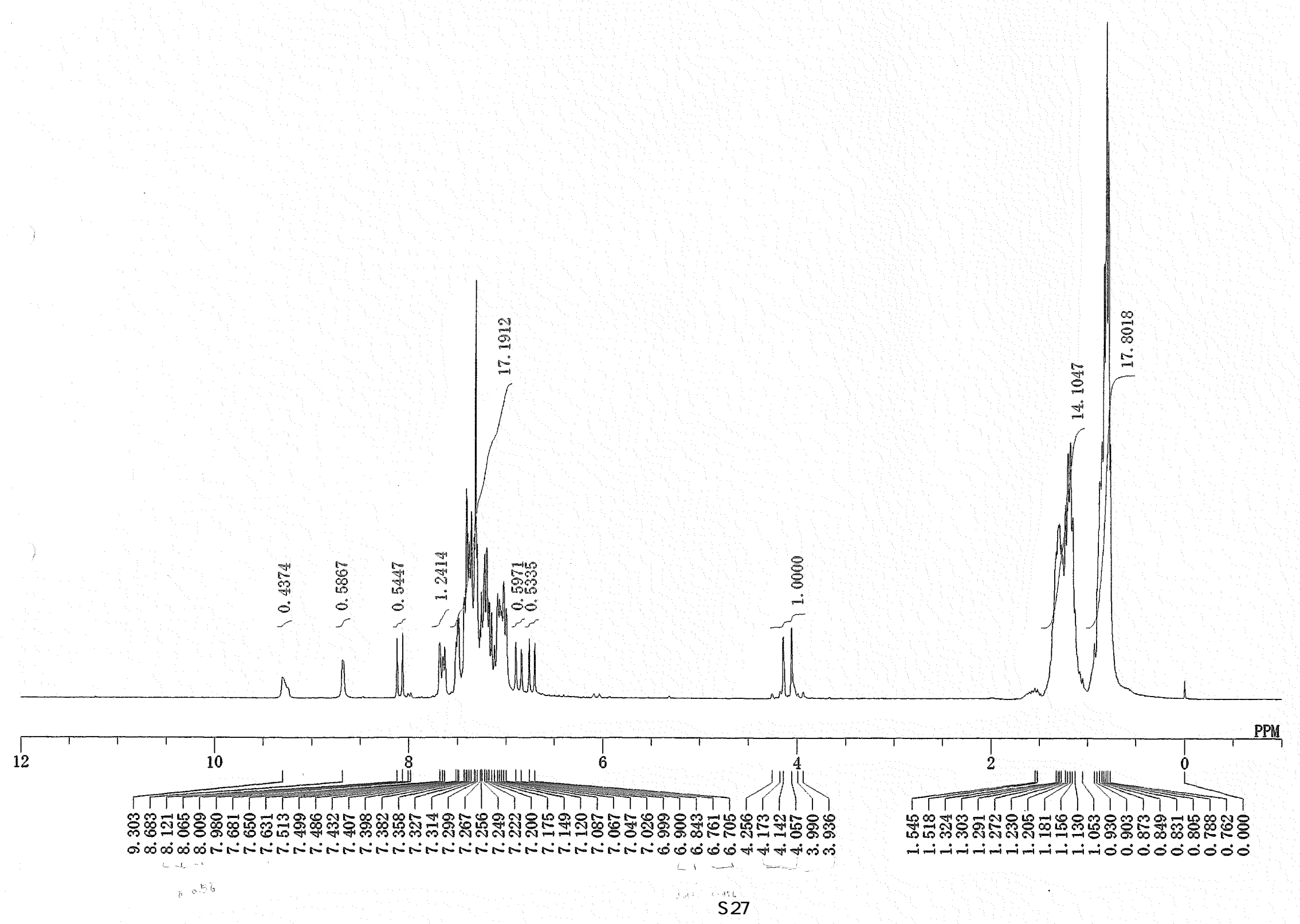

$\sum_{0}^{\mathrm{Sh}} \mathrm{H}_{\mathrm{S}}^{\mathrm{H}} \mathrm{P}_{\mathrm{S}}^{\mathrm{Ph}}$

1d 


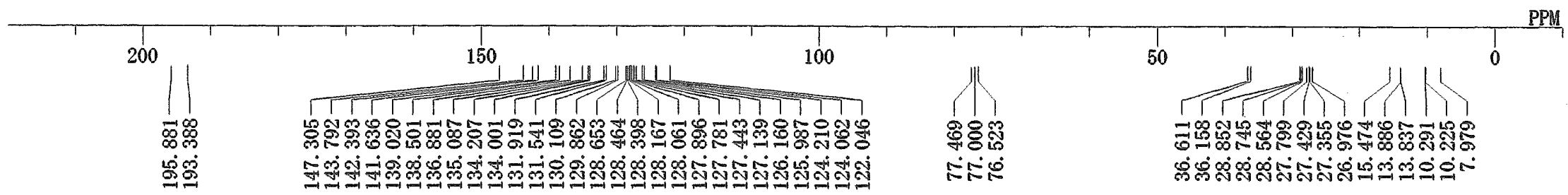


M2-OH-P124

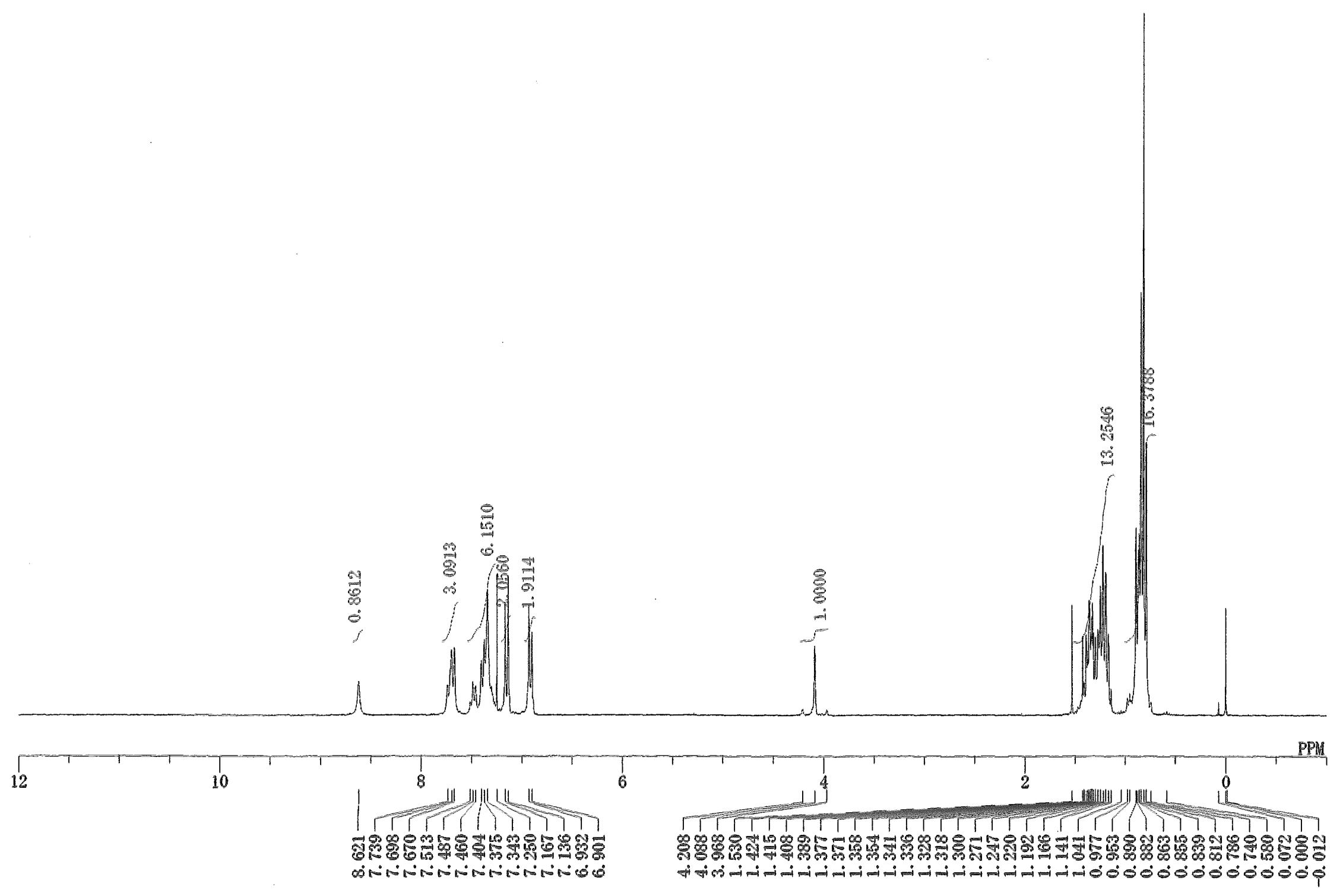




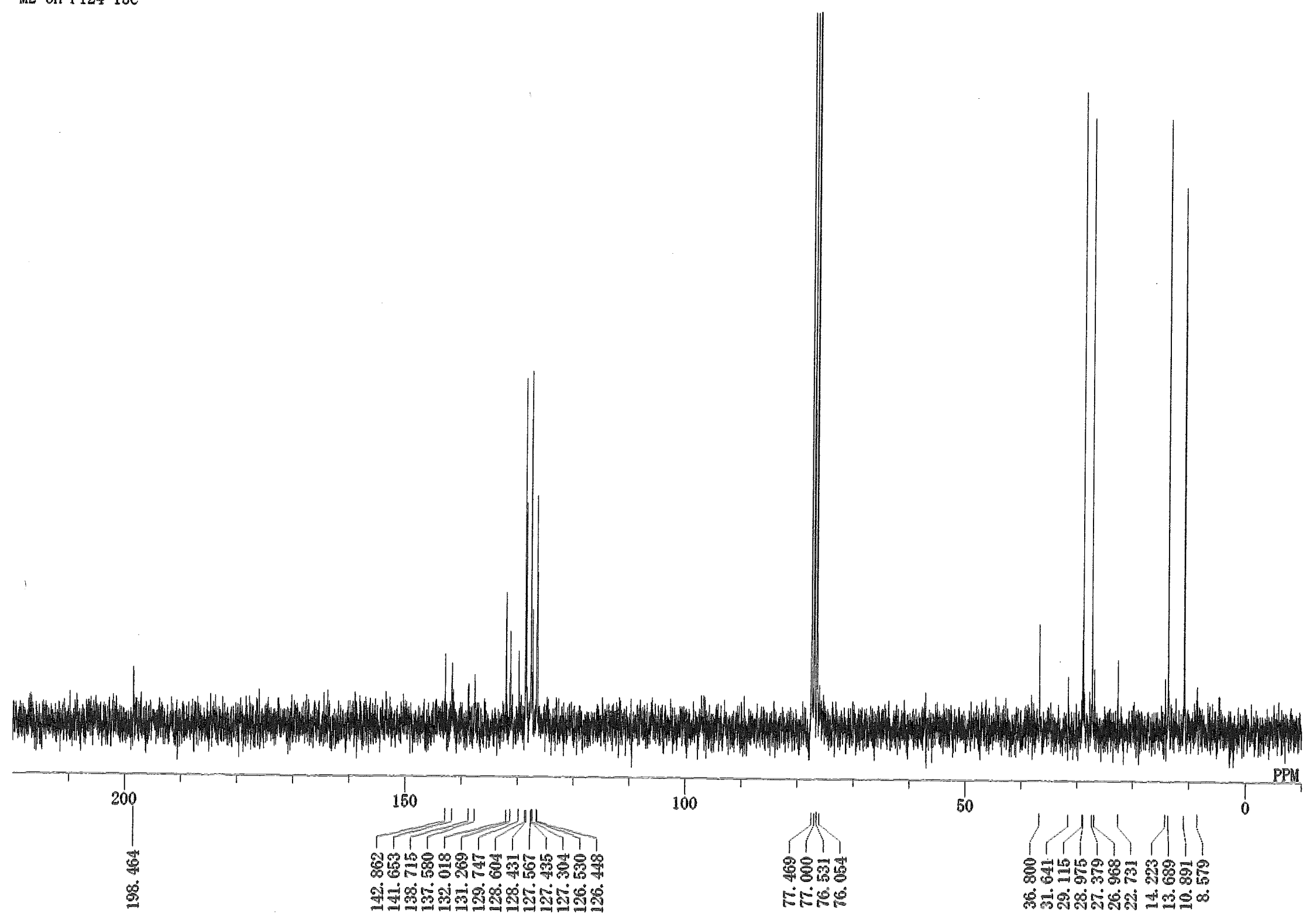


M2-OH-P73

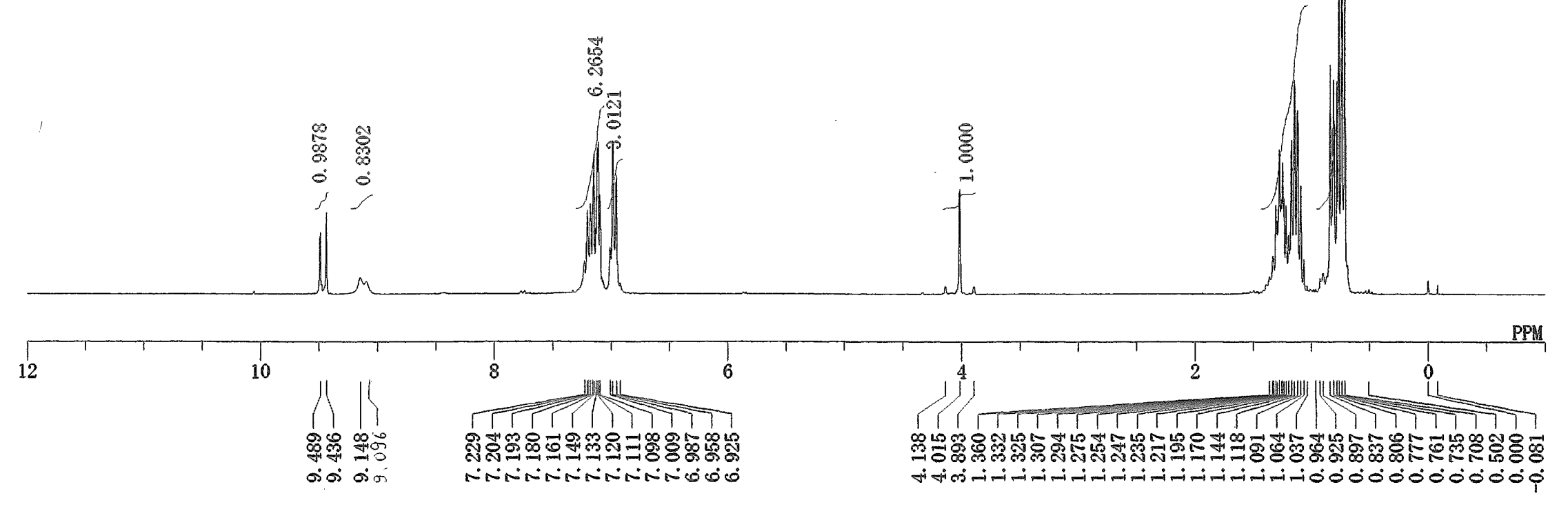




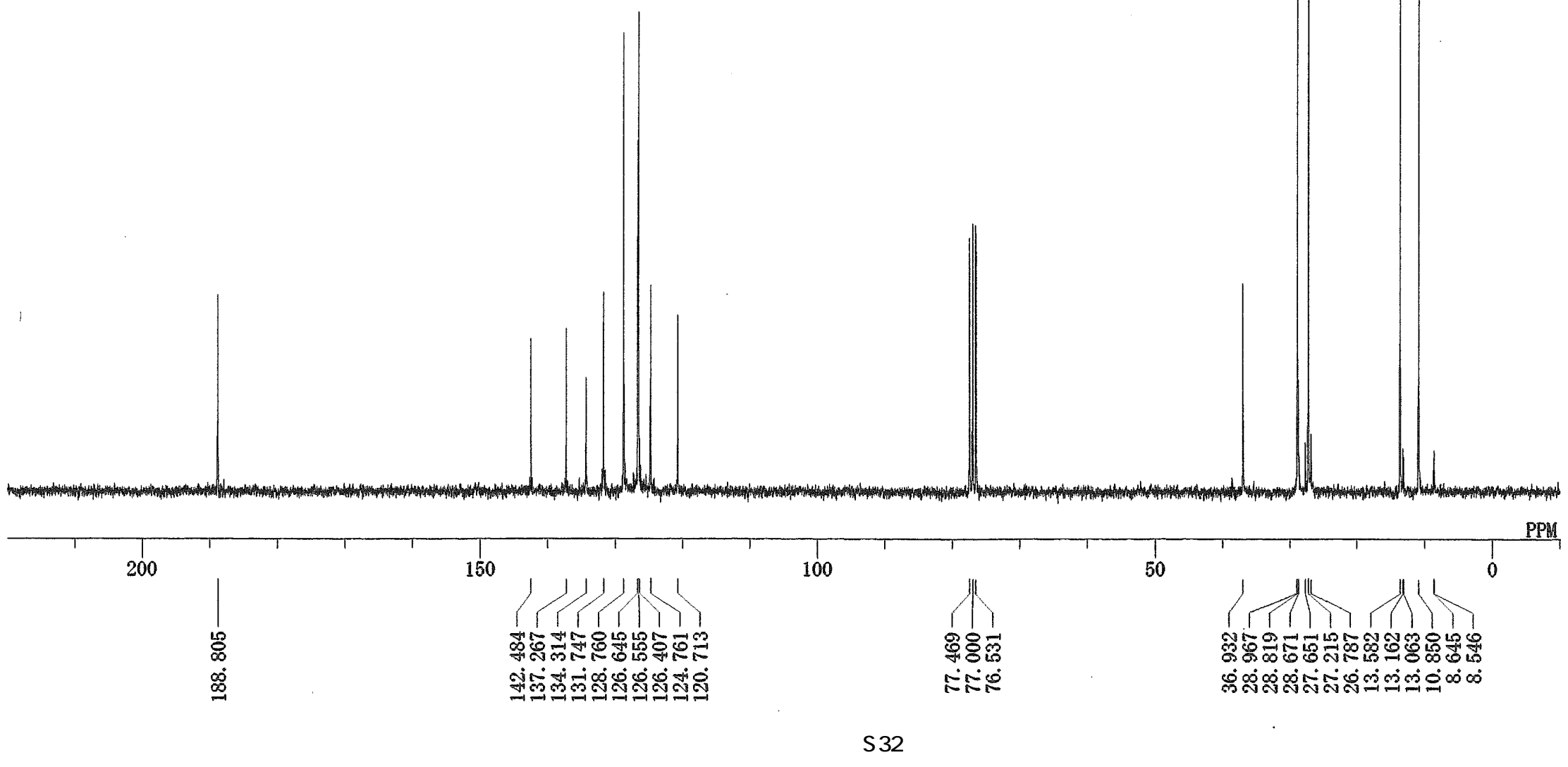


M1-OH-P50

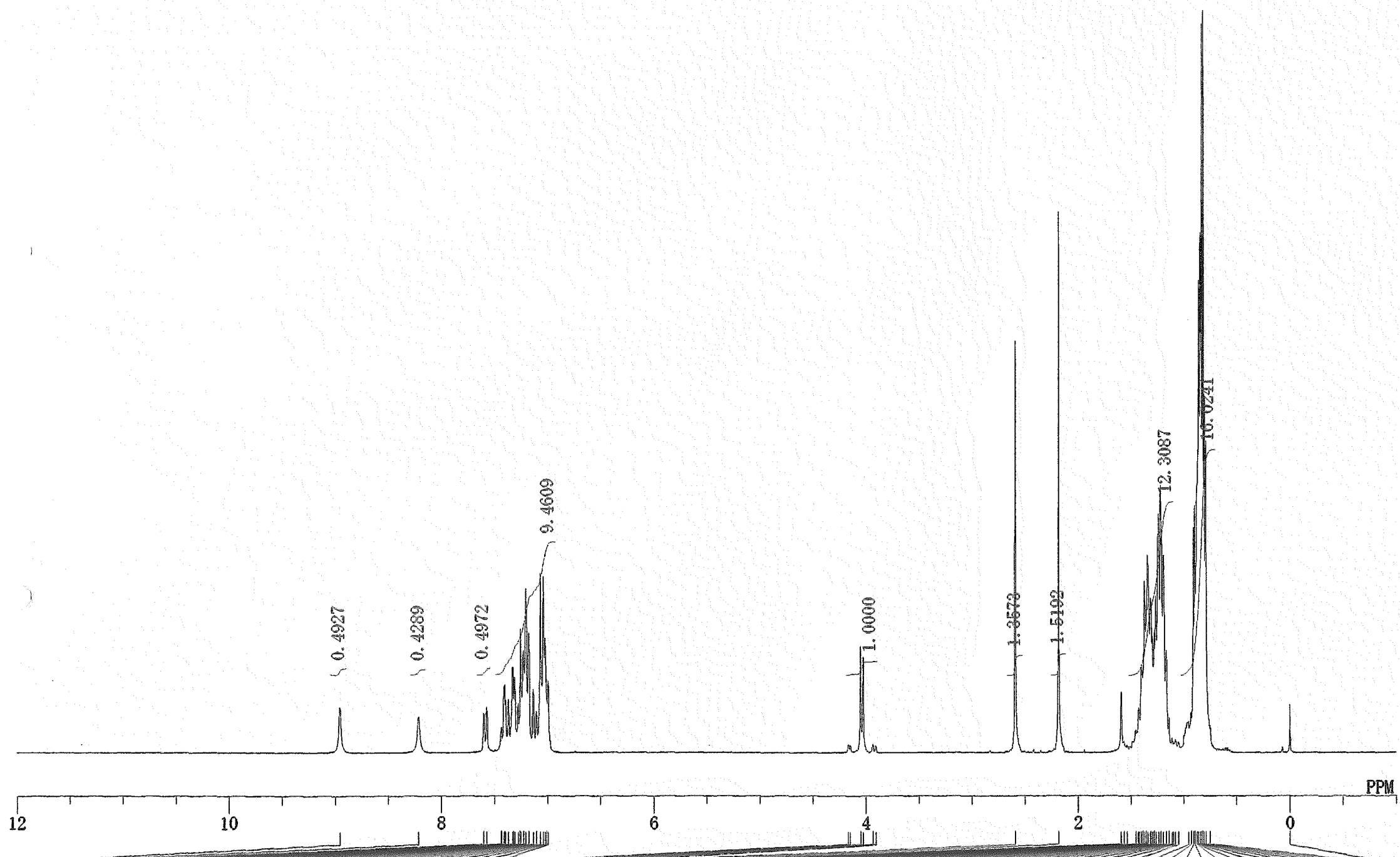

6011191 今. o
$\int_{\left[O T_{S}^{H}\right.}^{\mathrm{SnBu3}} \mathrm{He}$

$1 \mathrm{~g}$ 


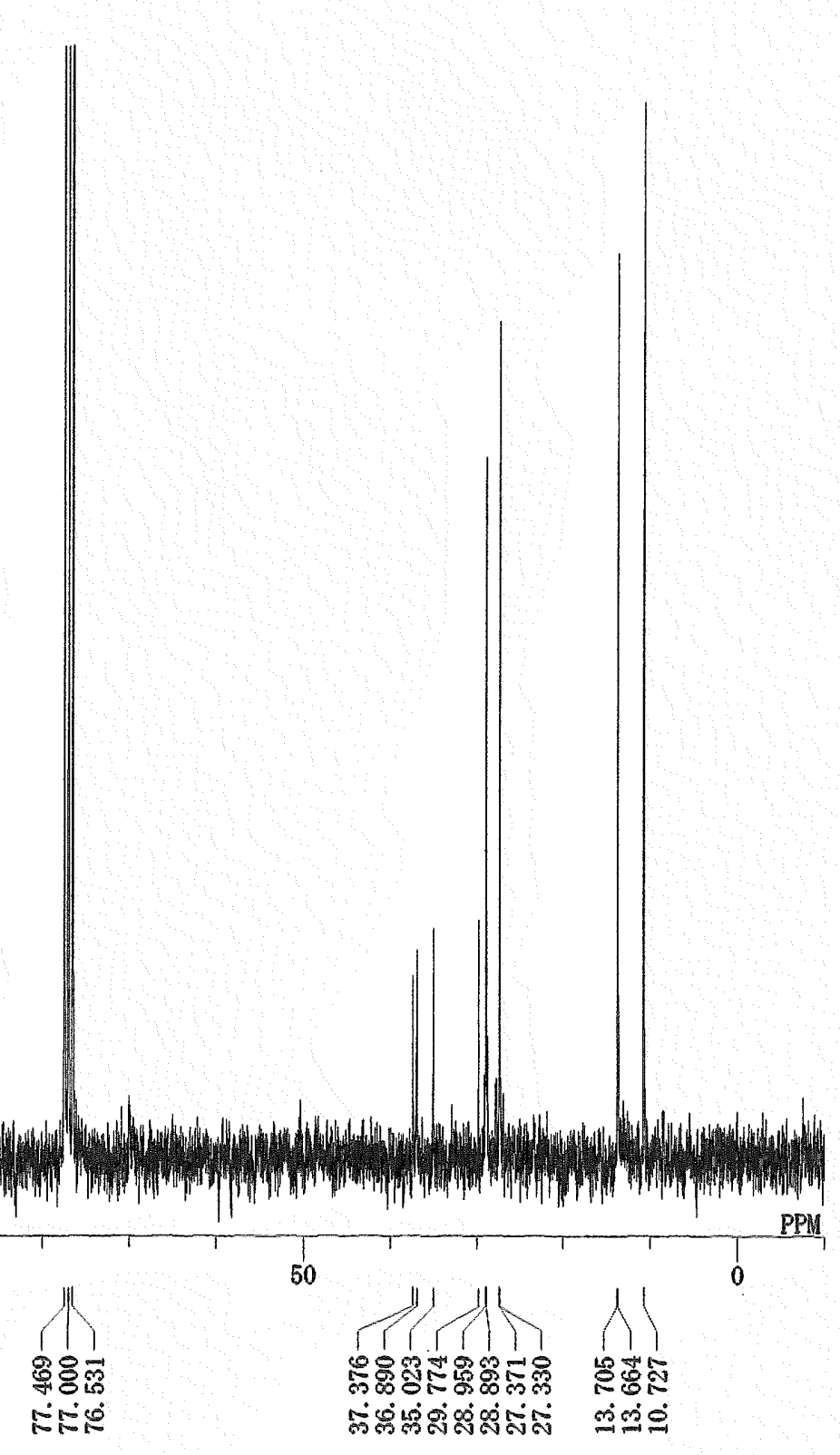

$\mathrm{CO}_{\mathrm{S}}^{\mathrm{H}} \mathrm{N} \mathrm{T}_{\mathrm{S}}^{\mathrm{Me}}$

$1 \mathrm{~g}$

P.

200

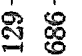

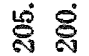

150

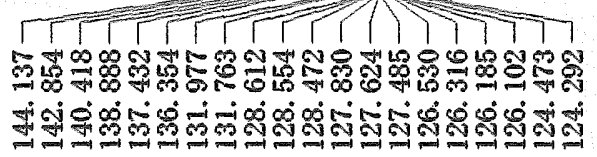

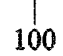

100 
M1-OH-P37

$\int_{0}^{\mathrm{H}} \pi_{5}^{\mathrm{Sn}}$

$1 \mathrm{~h}$

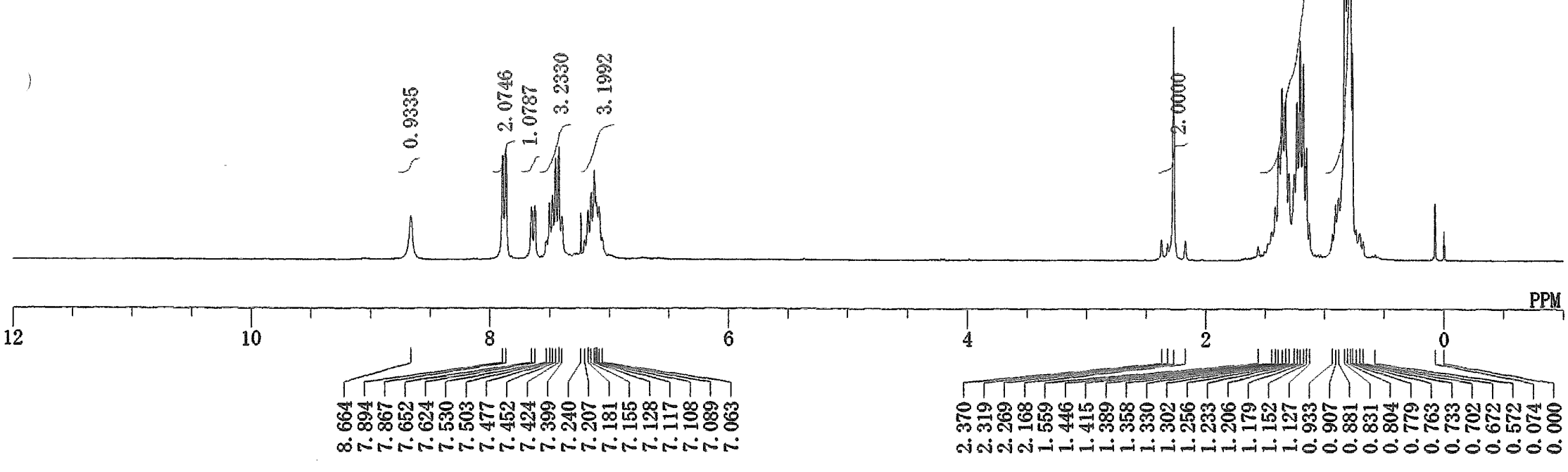


M1-OH-P37-13C

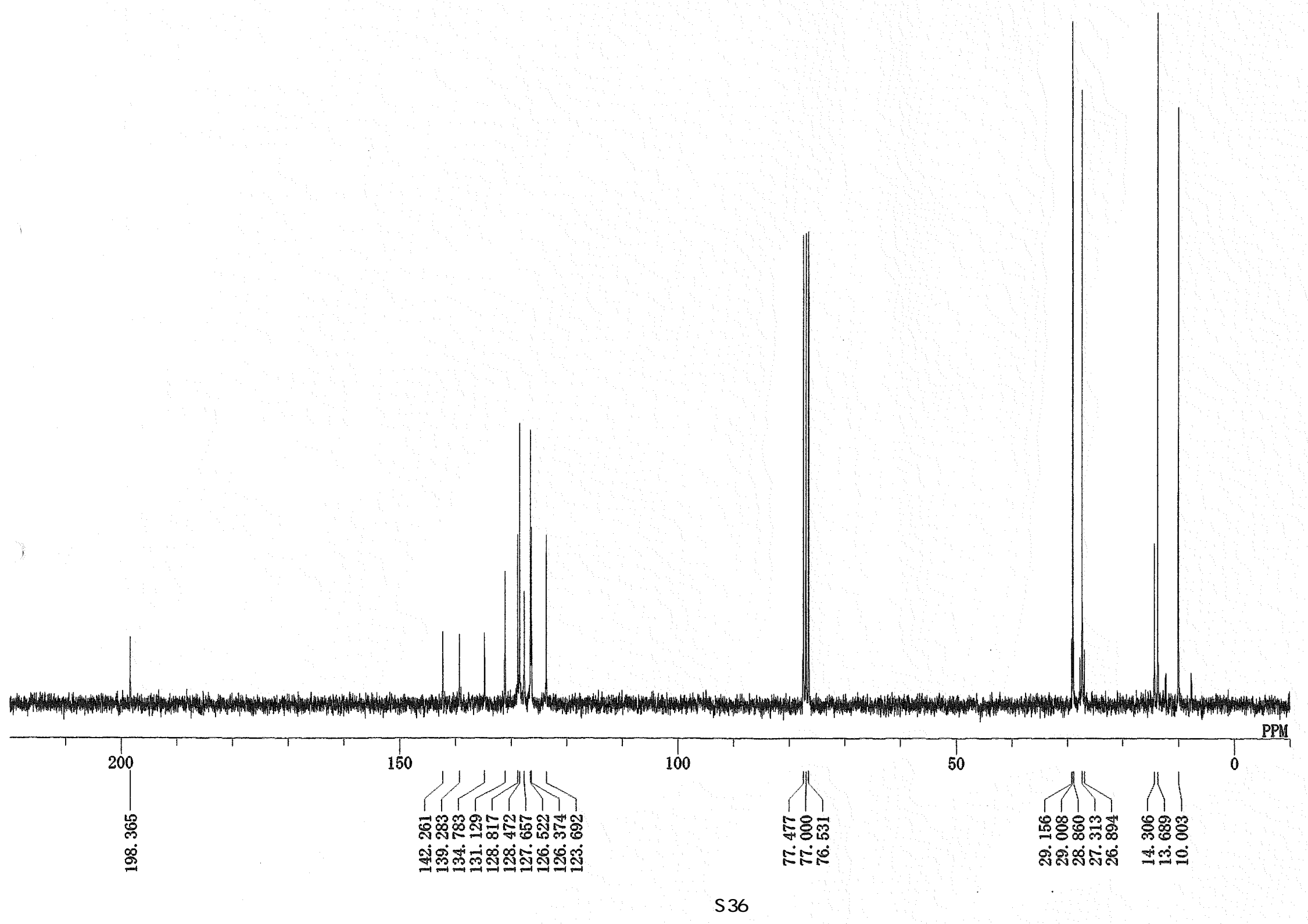


M1-OH-P7-fr6-13

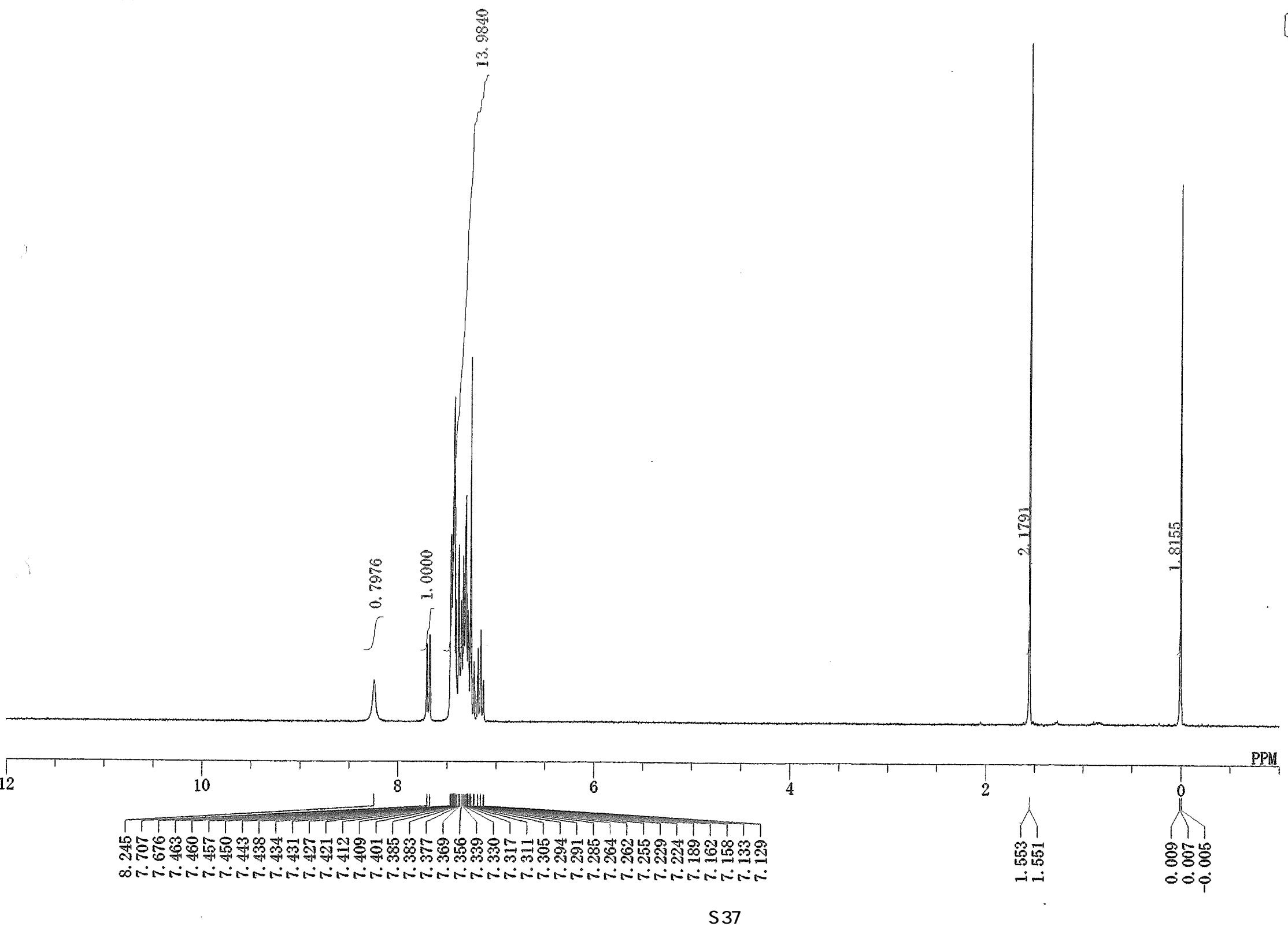

Q ${ }_{H}^{P h}$

$2 a$ 
M1-OH-P7-fr6-13-13C

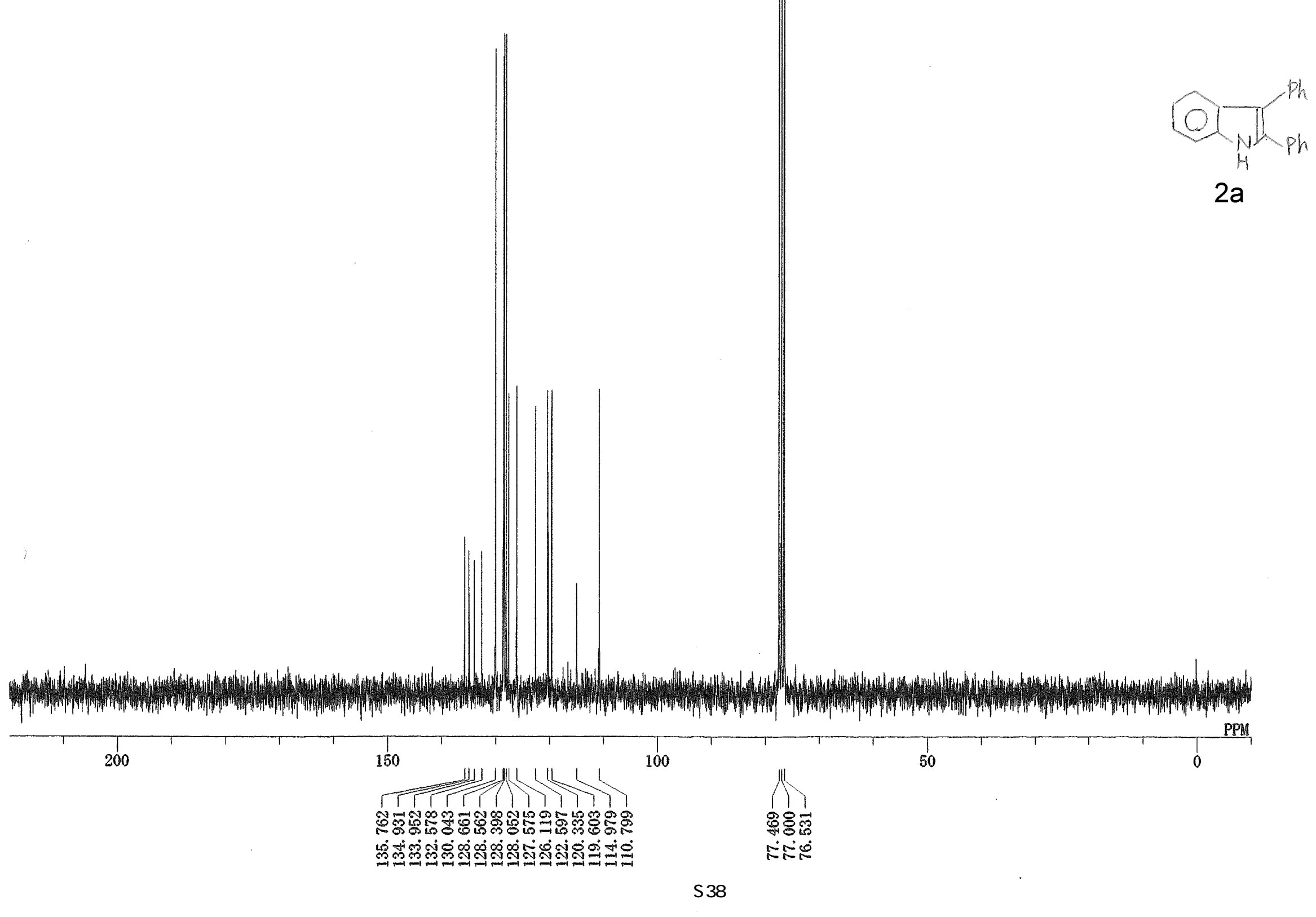


M1-OH-R47-fr8-12

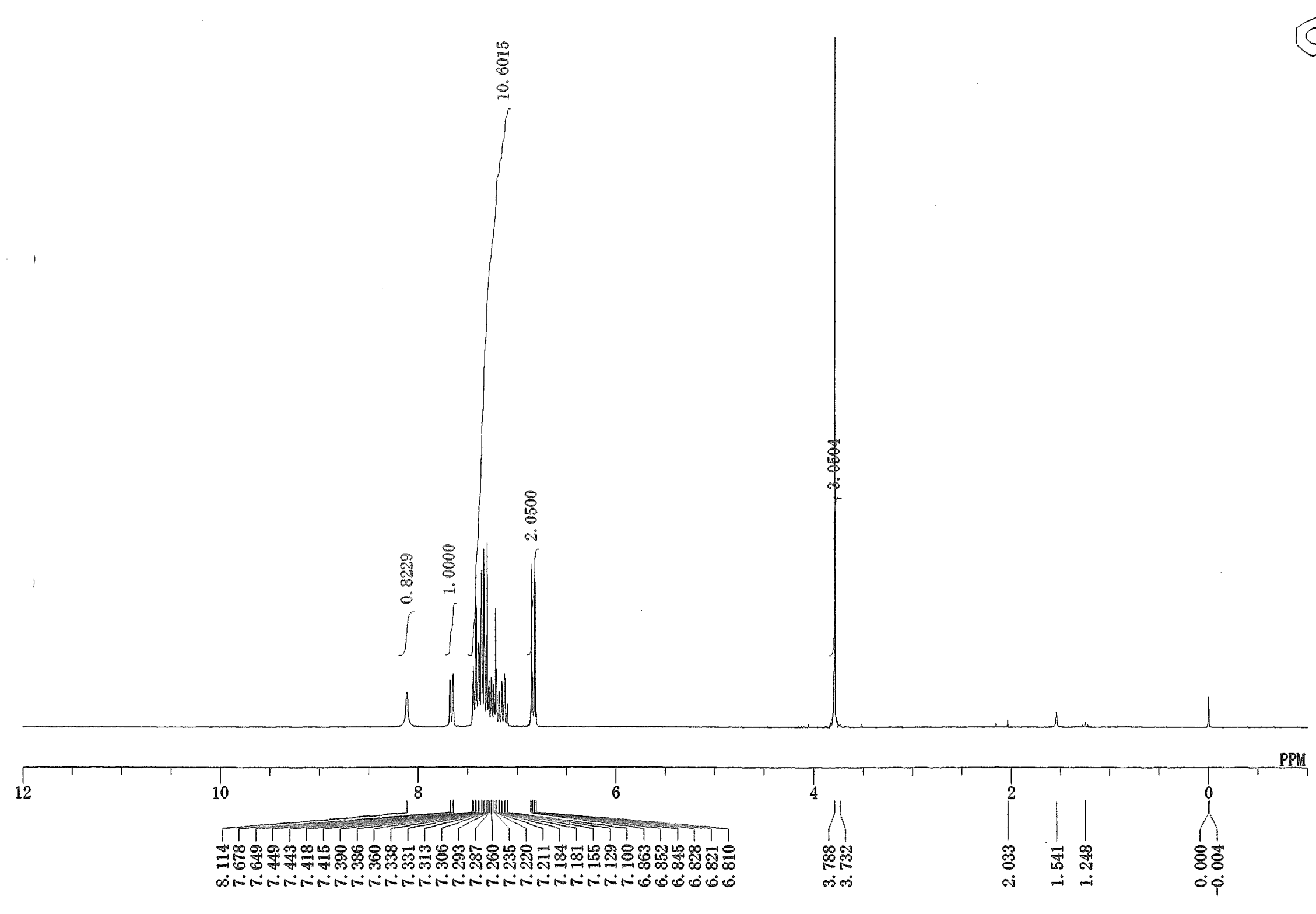


M2-0H-R47-13C

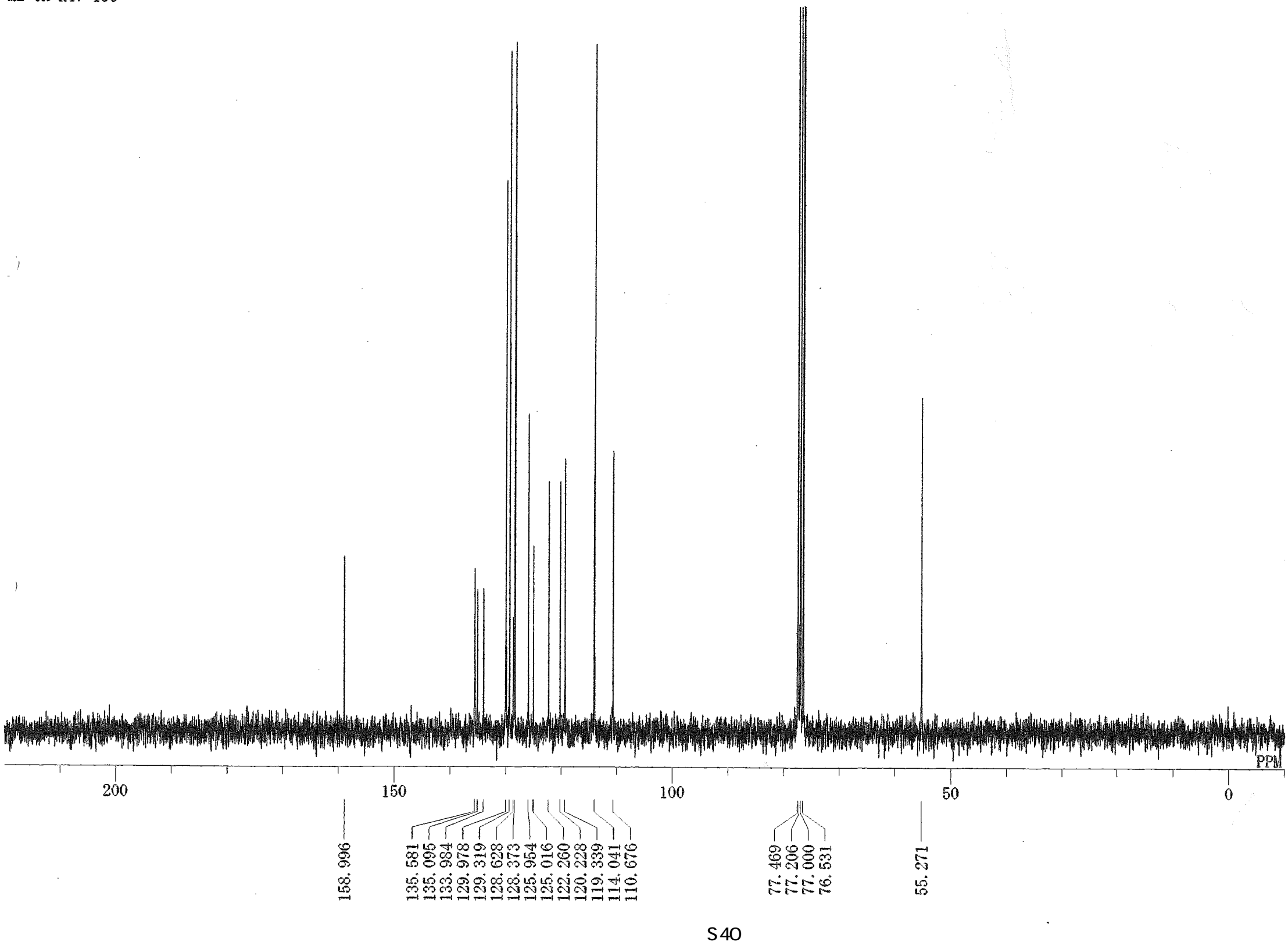

(0)

$2 b$ 
M1-0H-R50-fr5-13
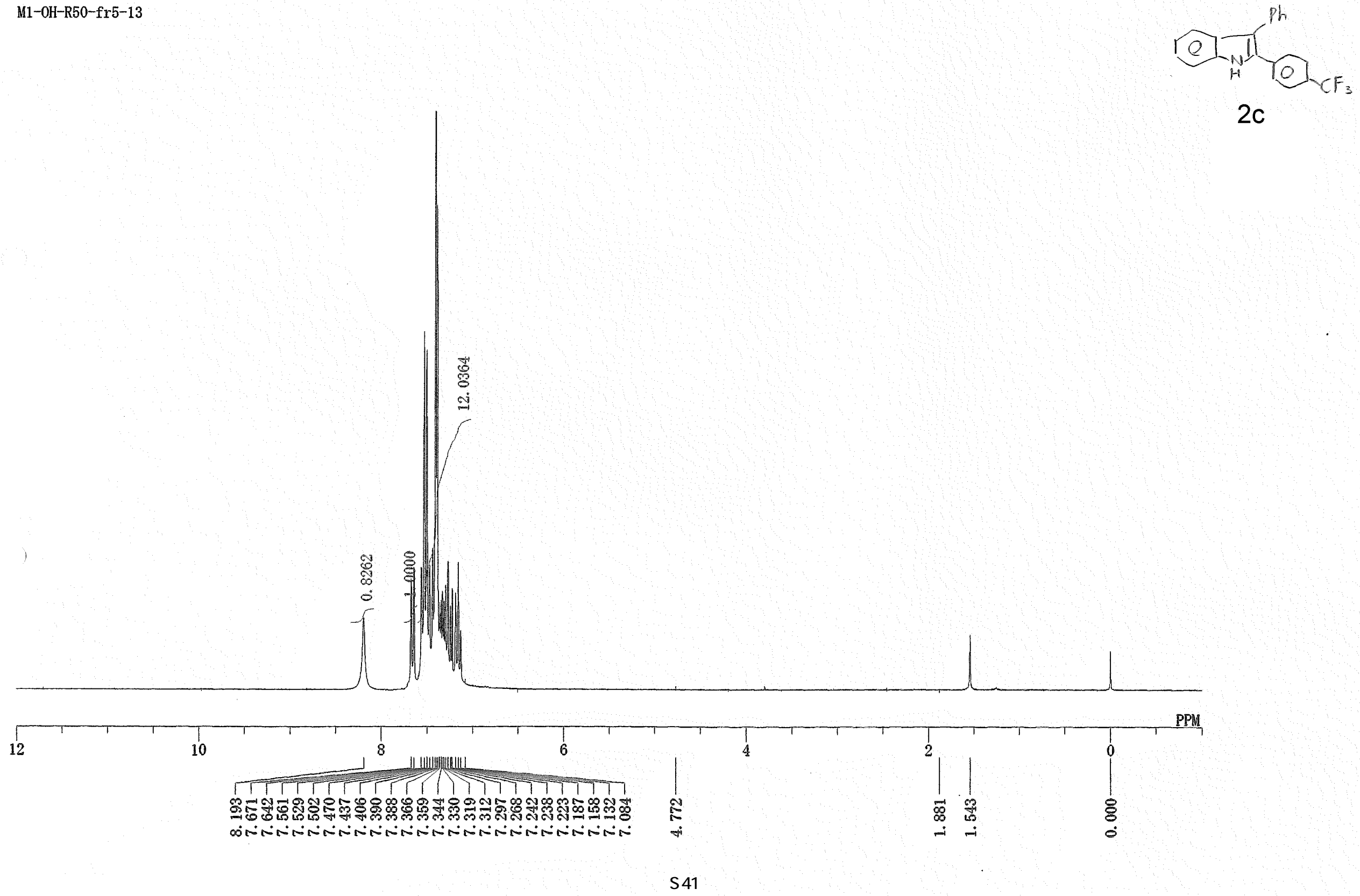


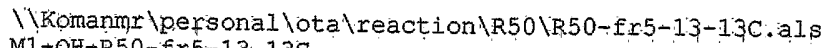

$\mathrm{M} 1 \rightarrow \mathrm{OH}-\mathrm{R} 50-\mathrm{f}$

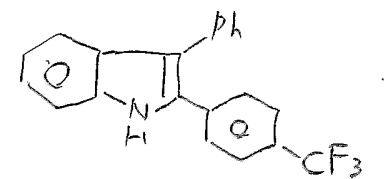

2c

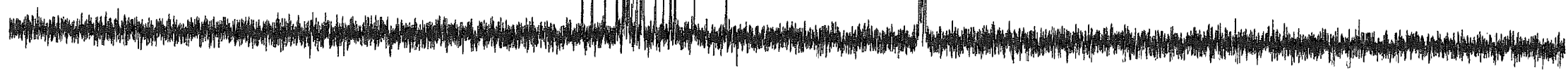

200

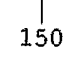

U1]

100

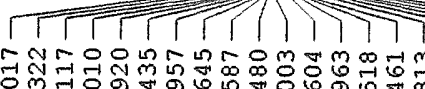

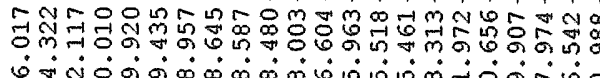

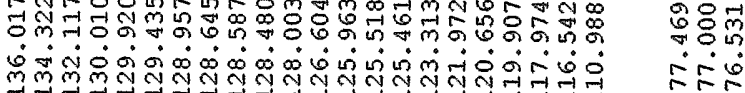

$\Delta \Delta \Delta \triangle 0$ S42

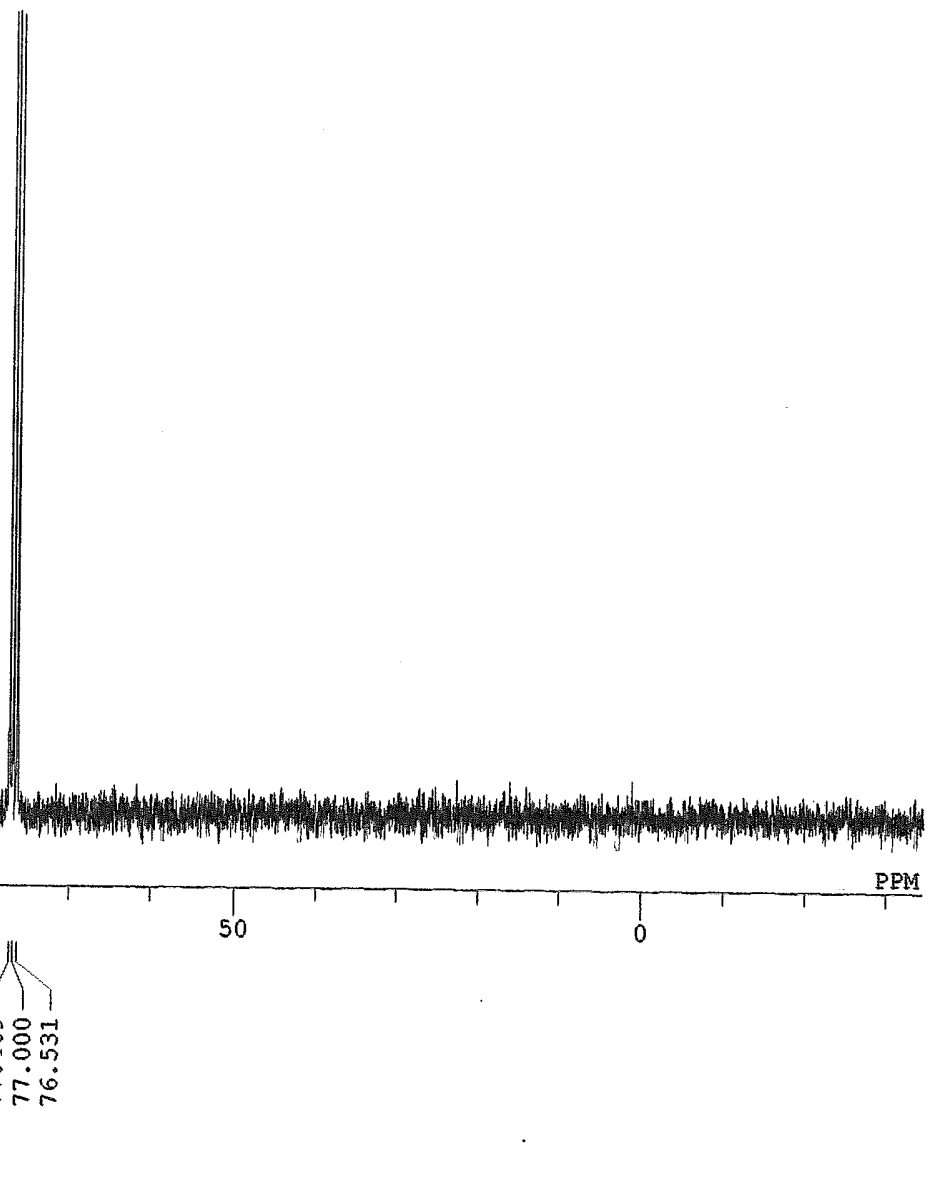


M2-OH-R72

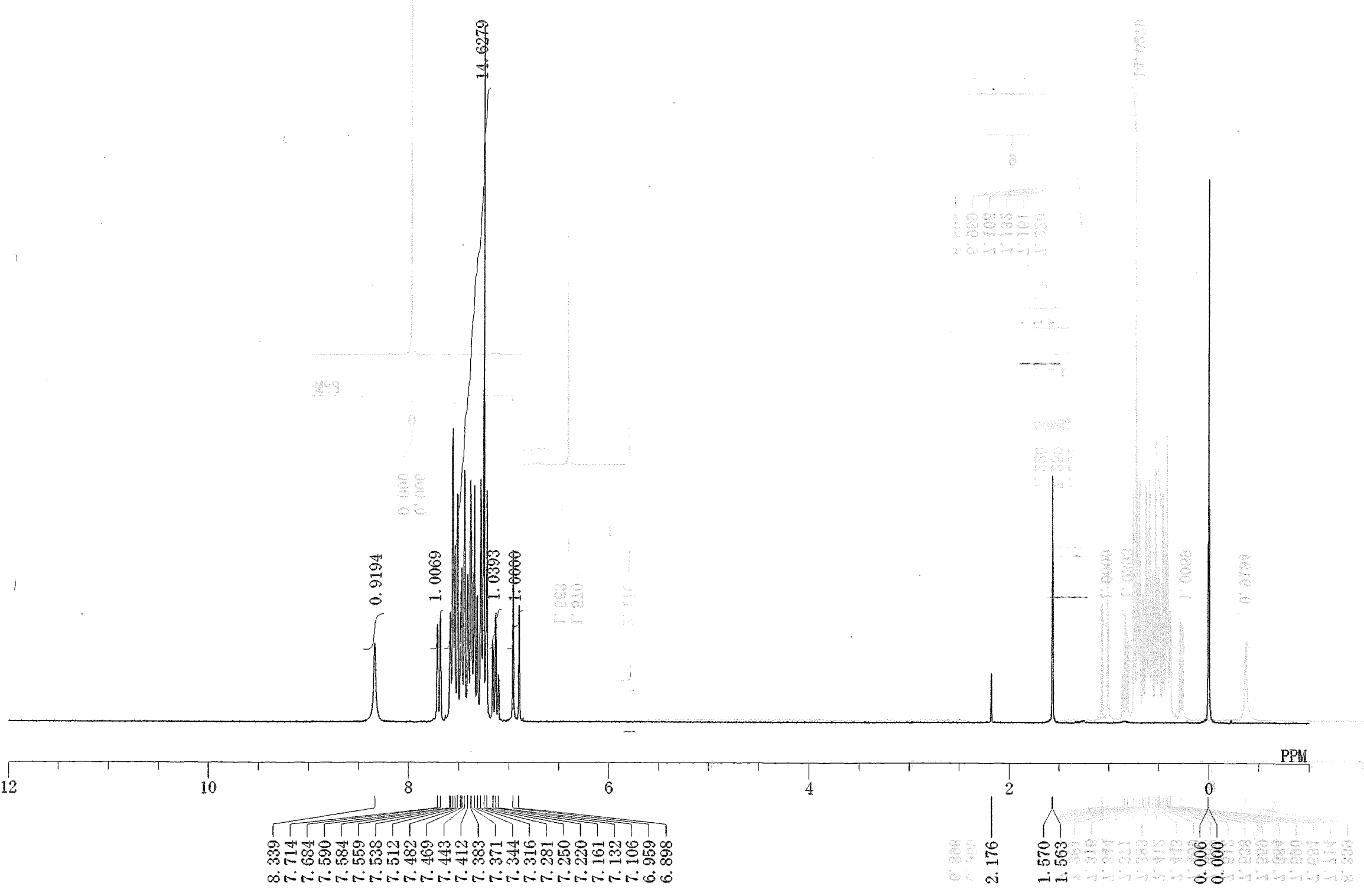

ODn

$2 d$ 


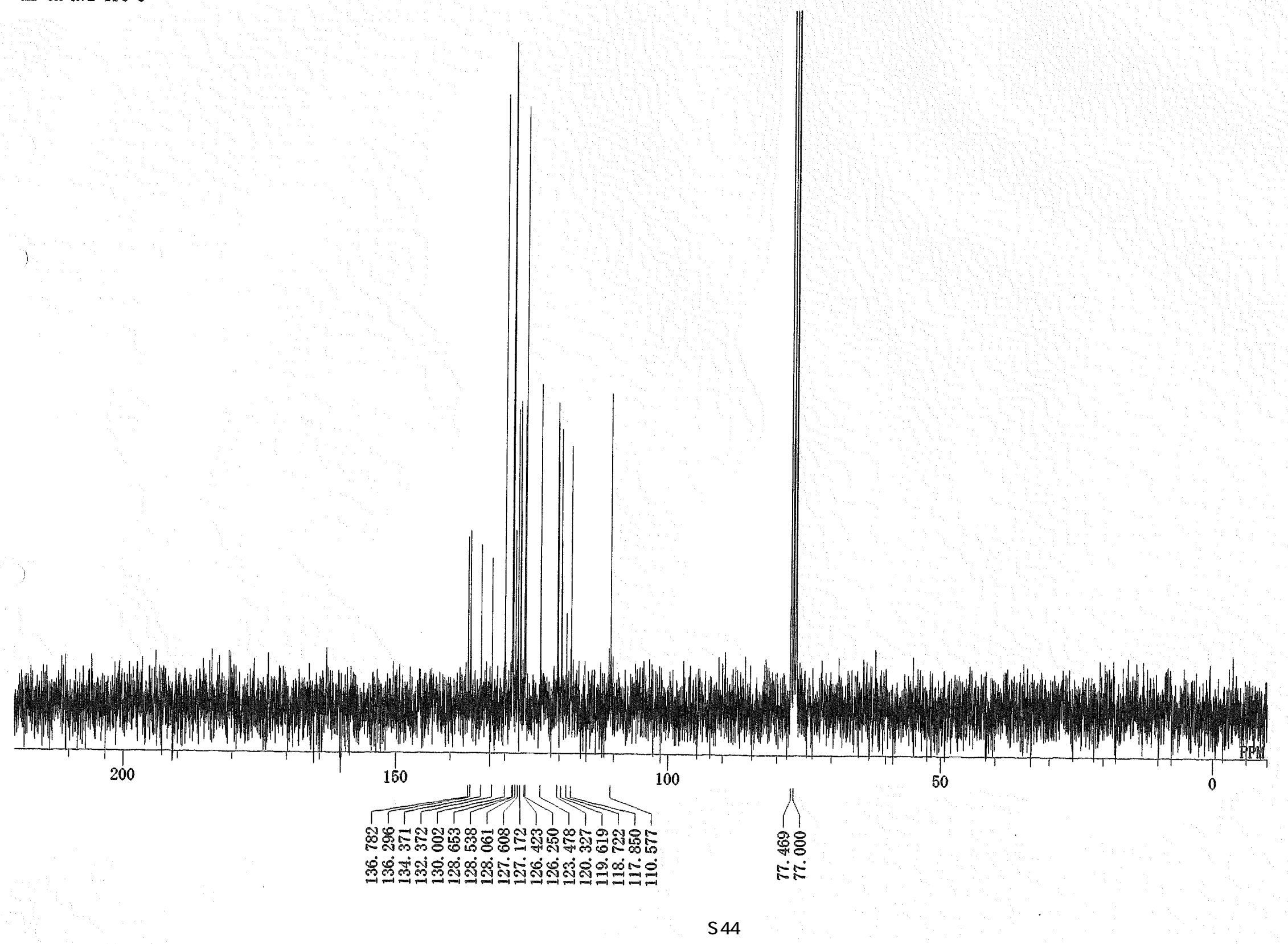


M2-OH-R88-fr6-15

(0) Or $^{4}$

$2 e$

兽

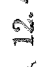

12

10

8

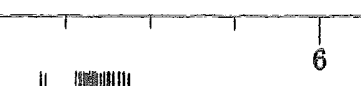

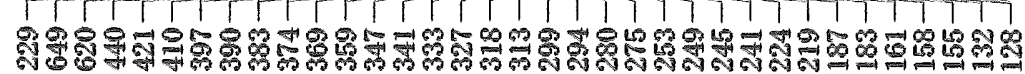

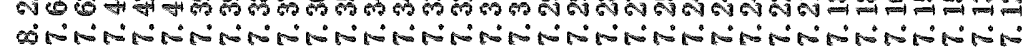

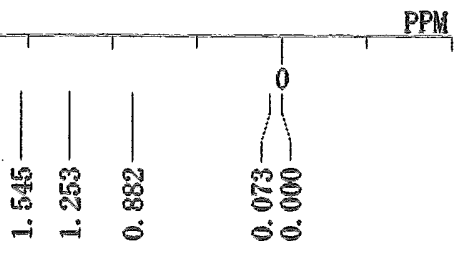


M2-0H-R88-fr6-16-13C

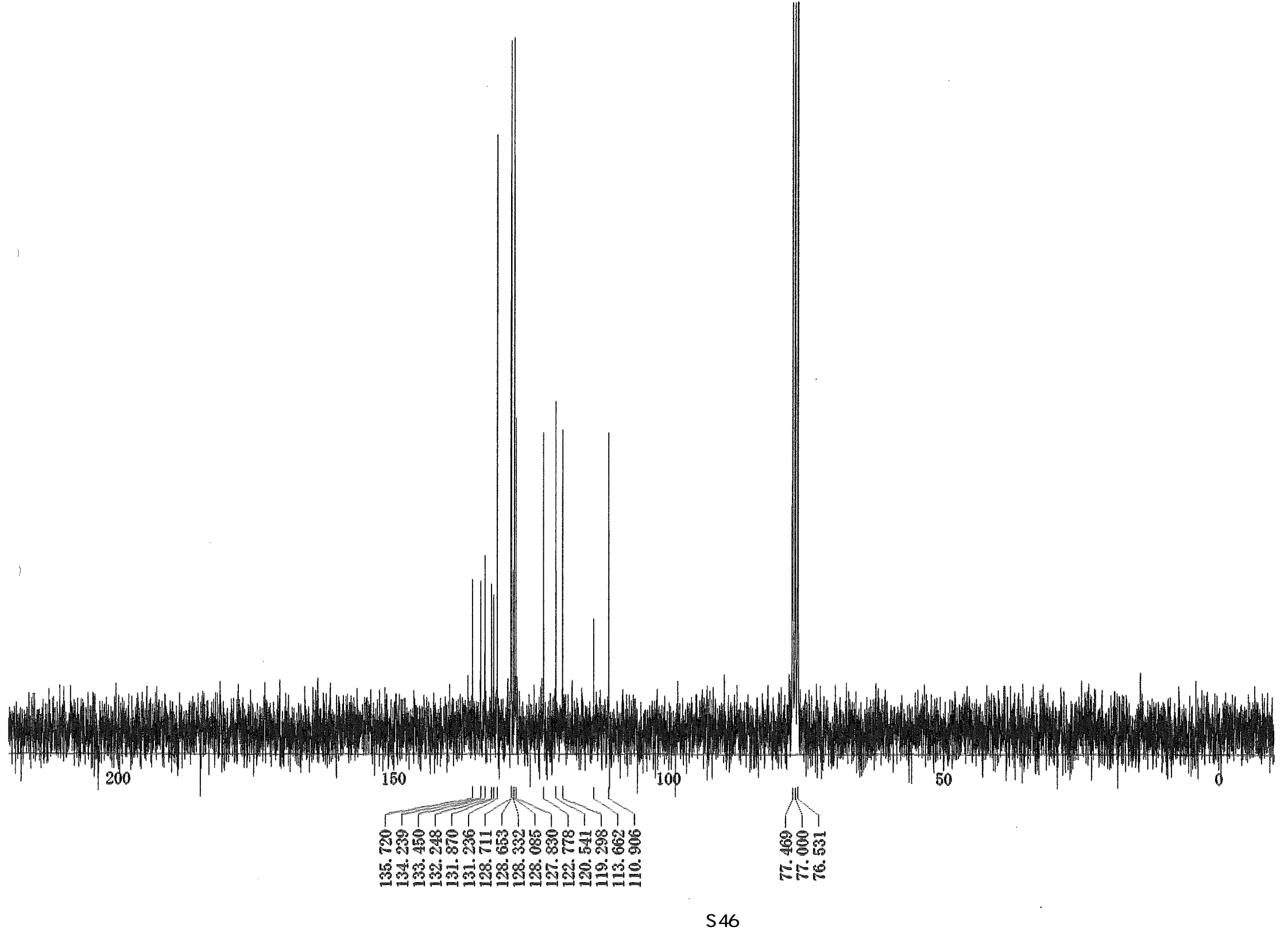

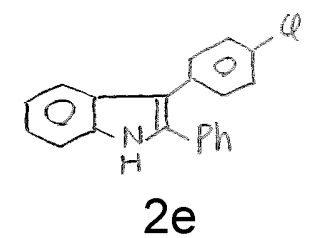


M2-OH-R65-fr14-19-GPC-3

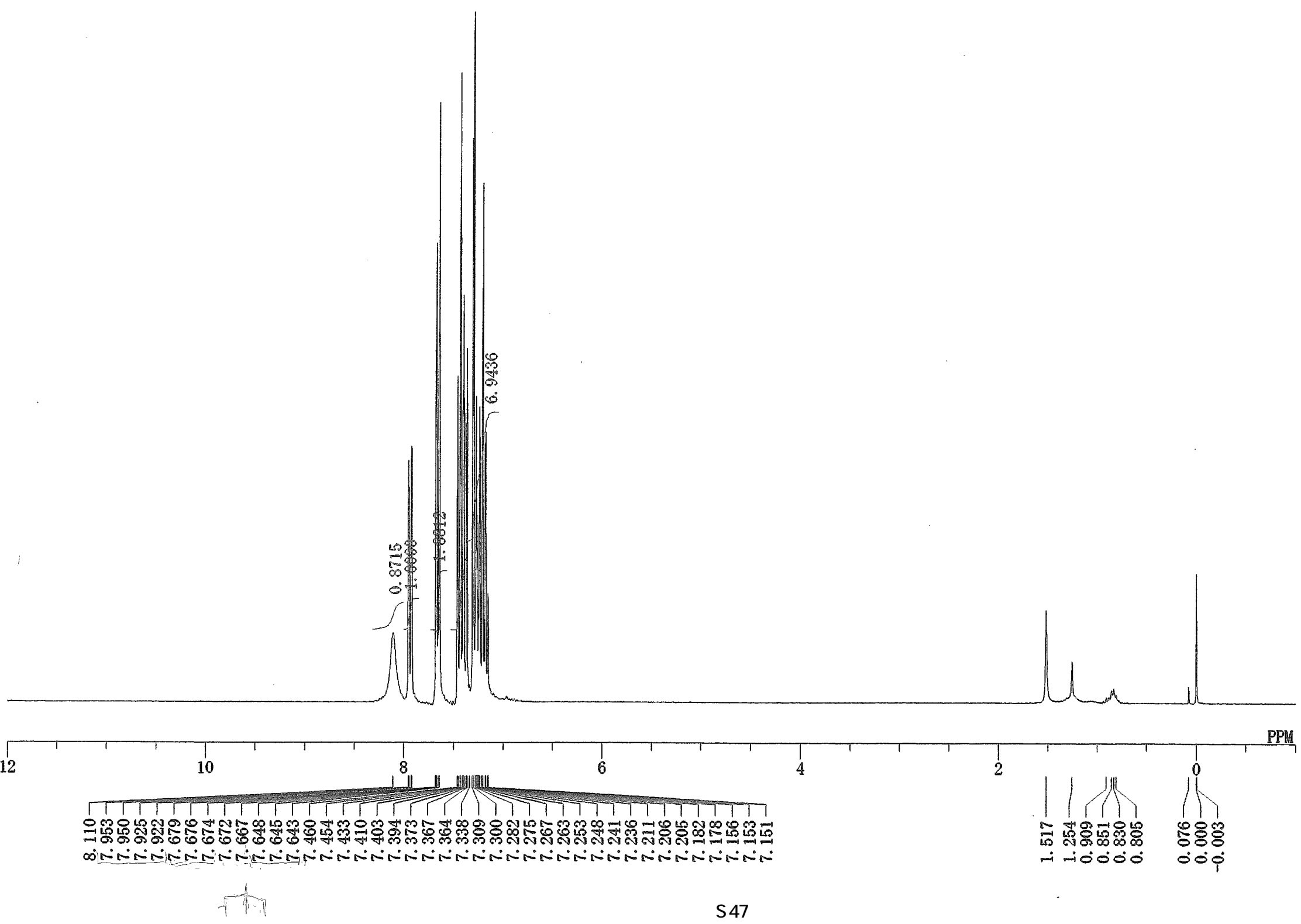


M2-OH-R65-fr14-19-GPC-3-13C

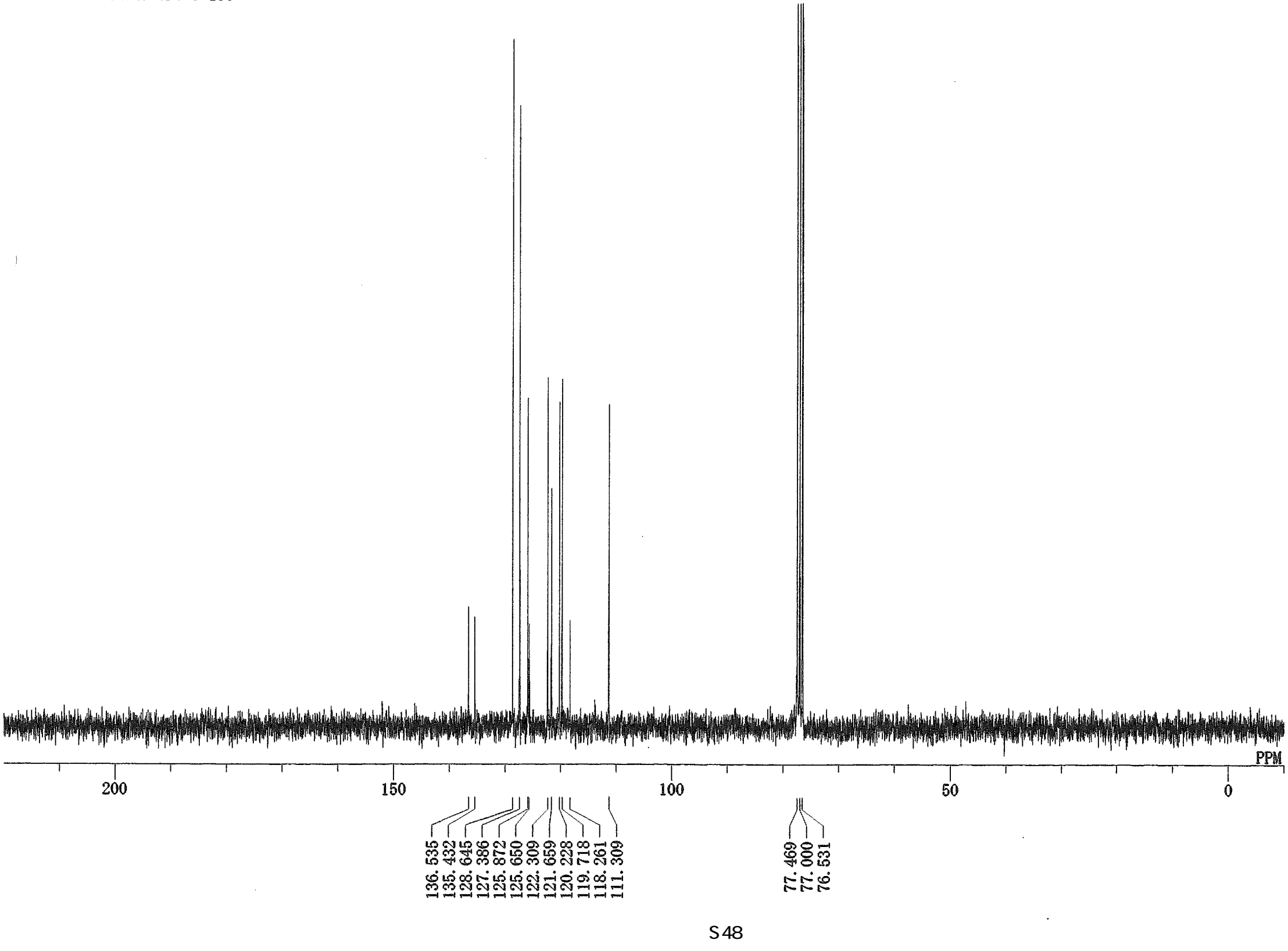

(0) 


\section{M1-0H-R55-fr9-12-GPC}

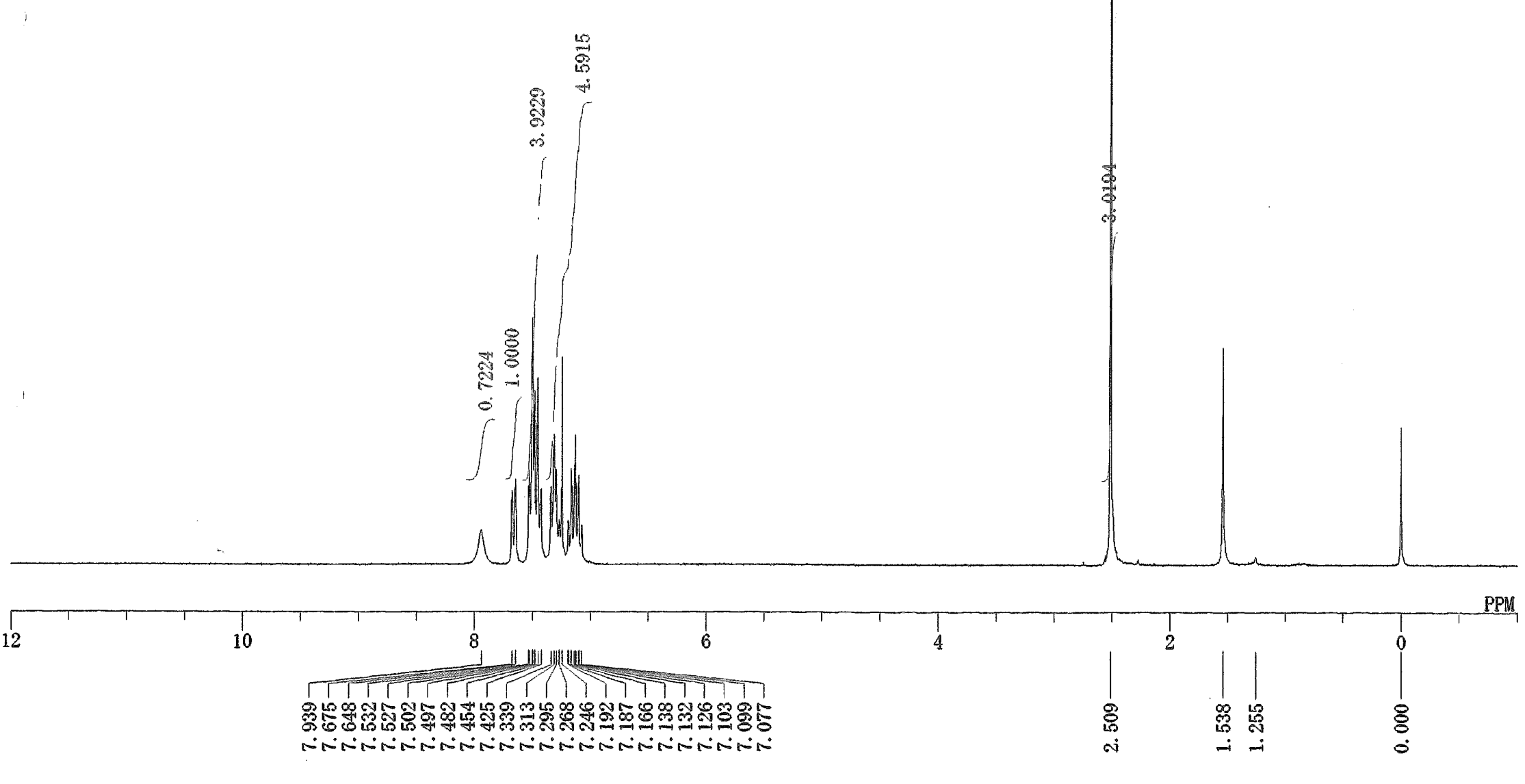




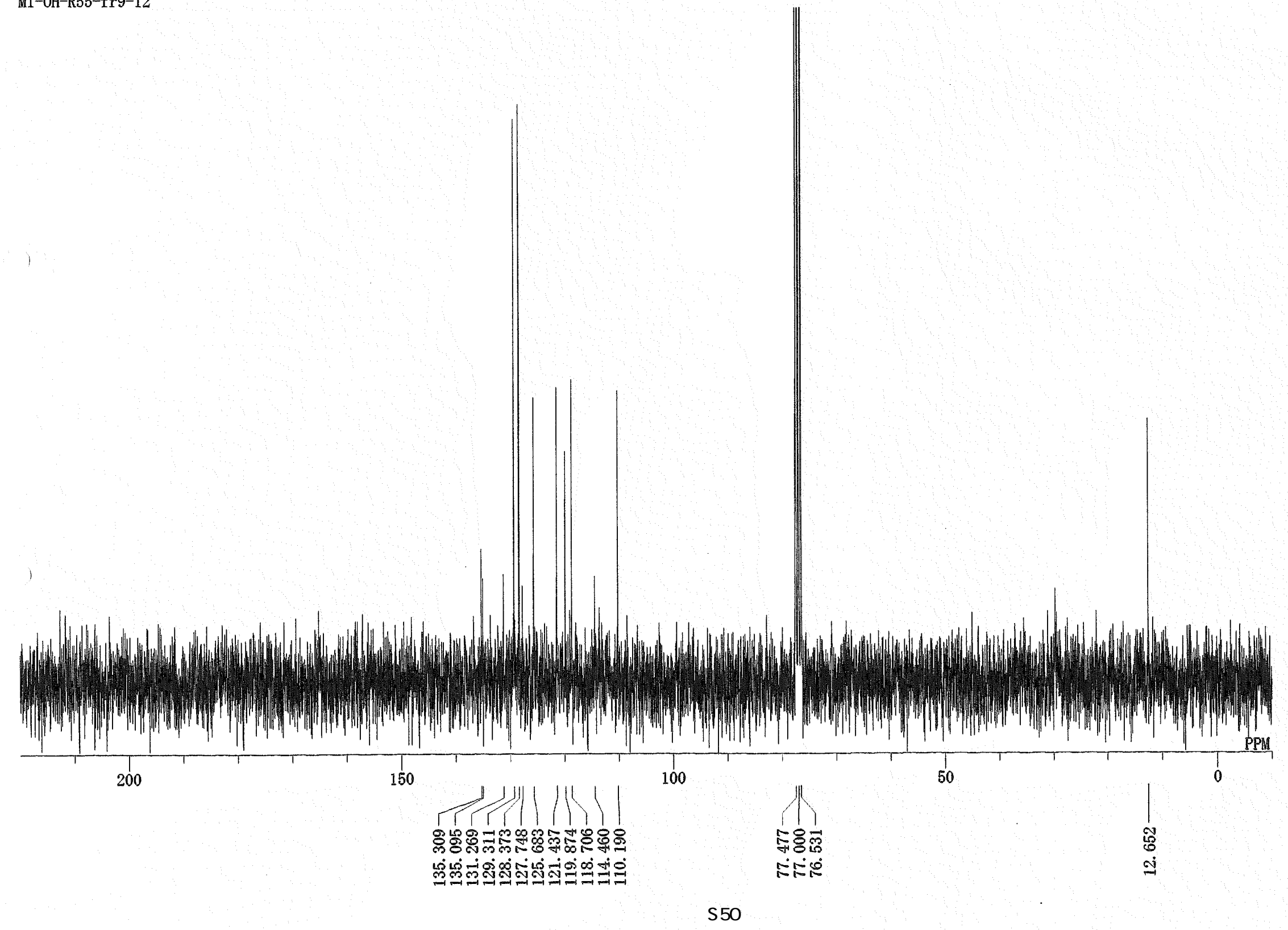


M1-OH-P23-indole

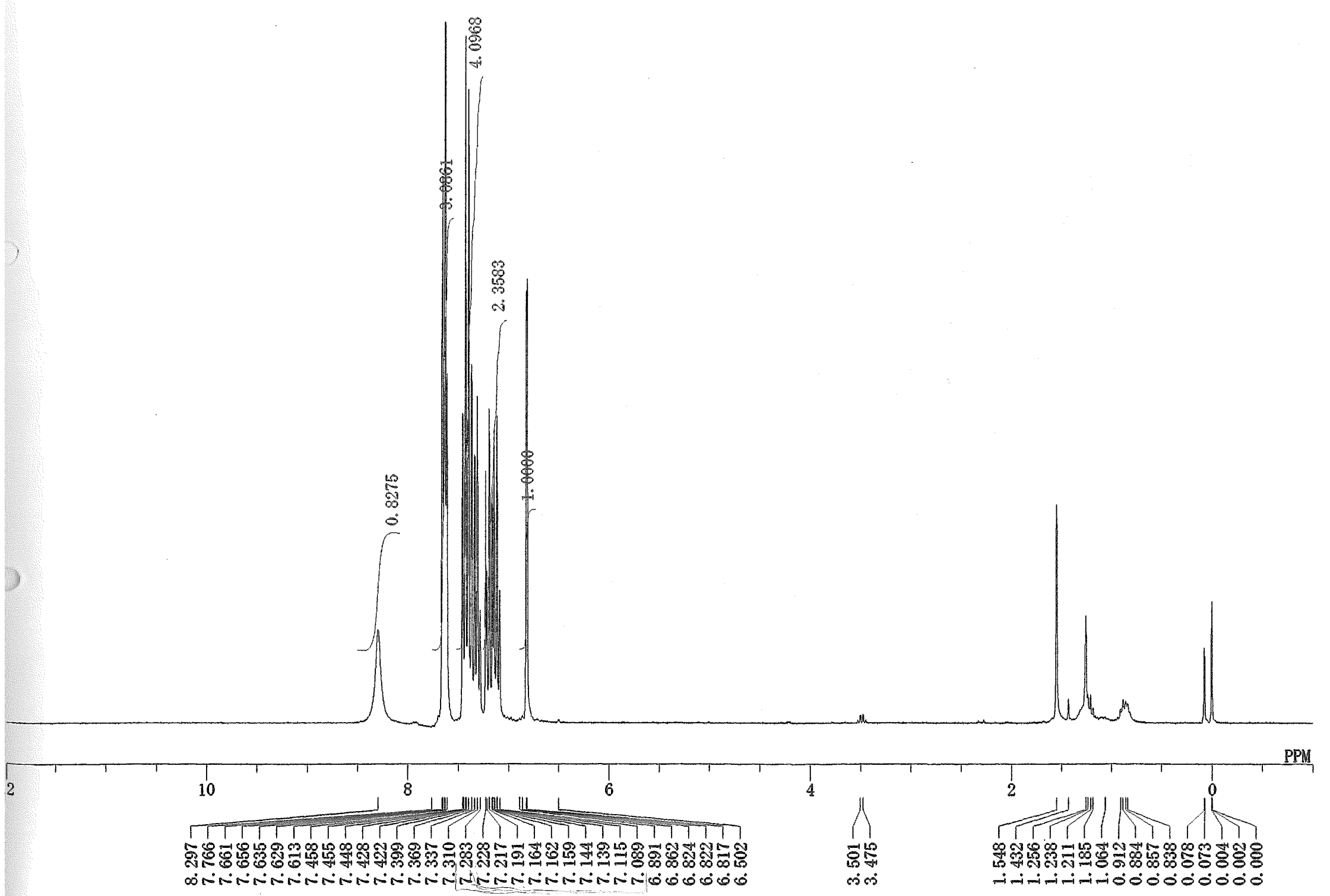

ofeph
$2 h$ 
M1-OH-P23-indole

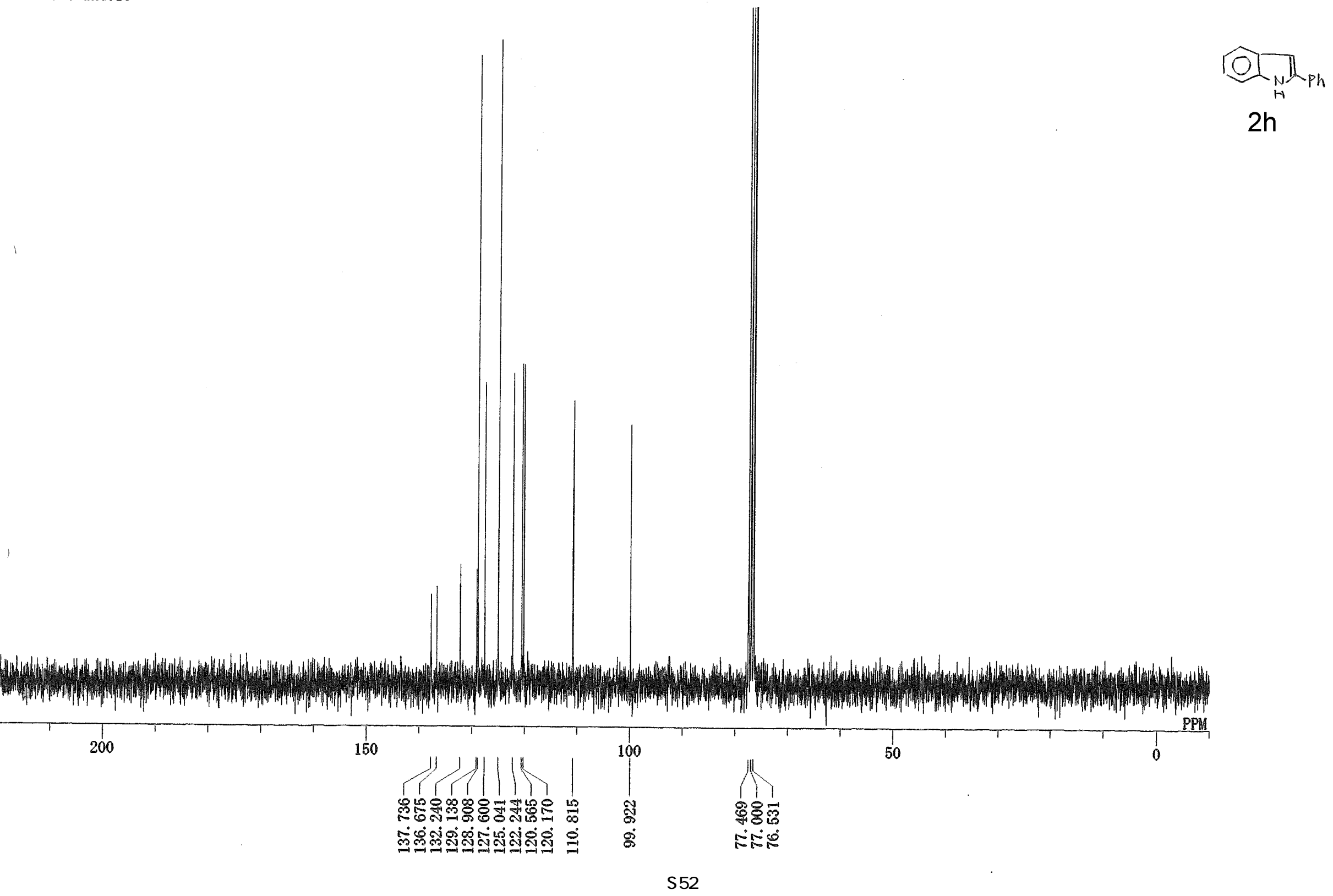




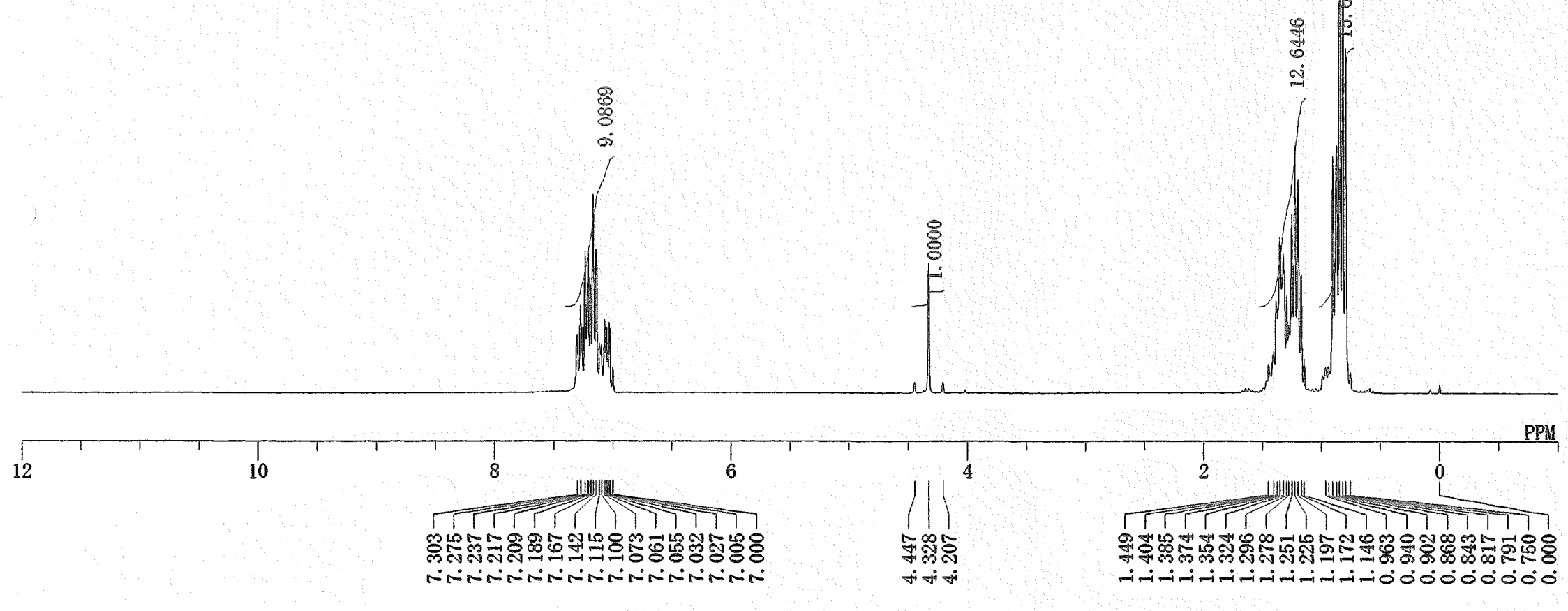




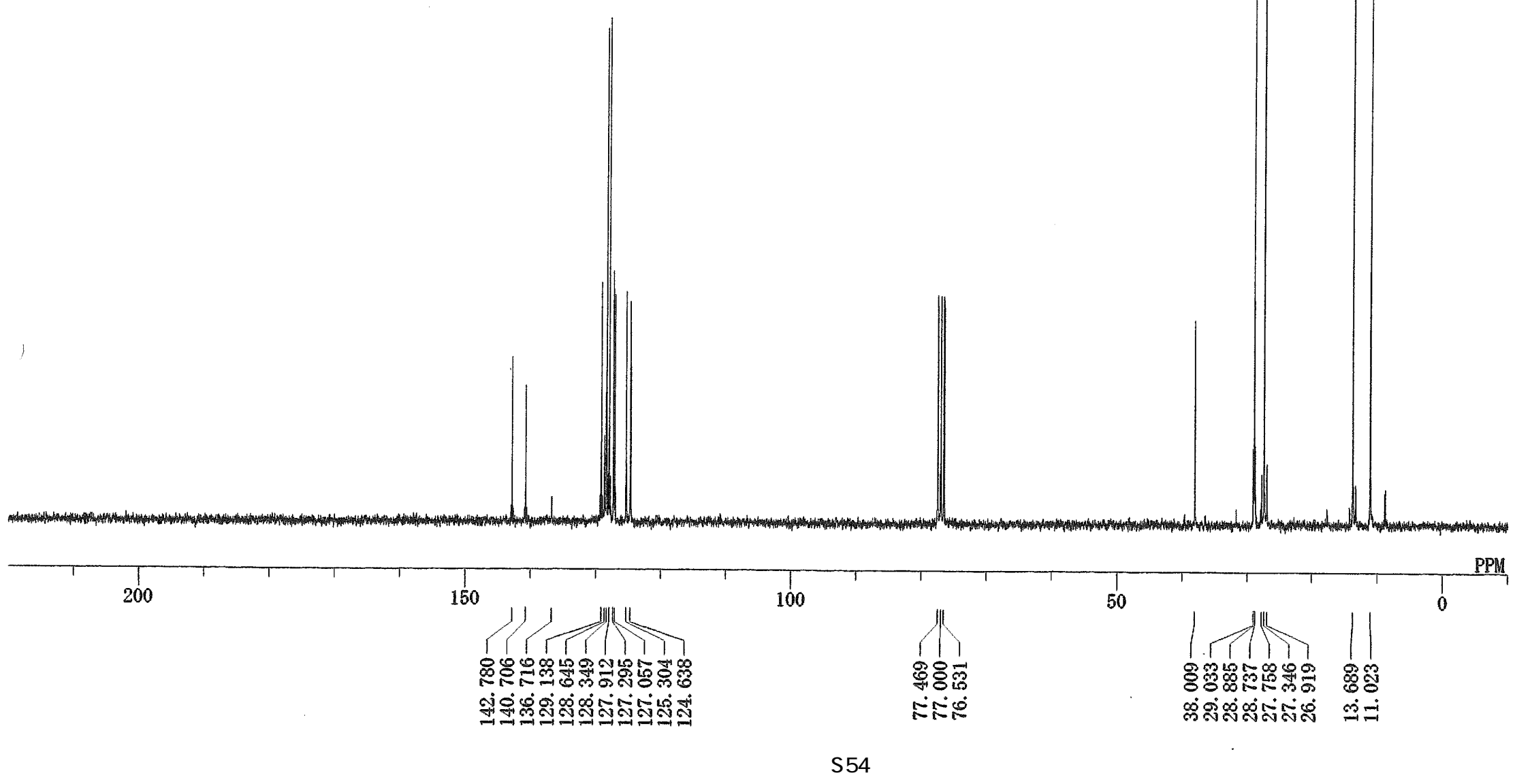


M2-OH-P103

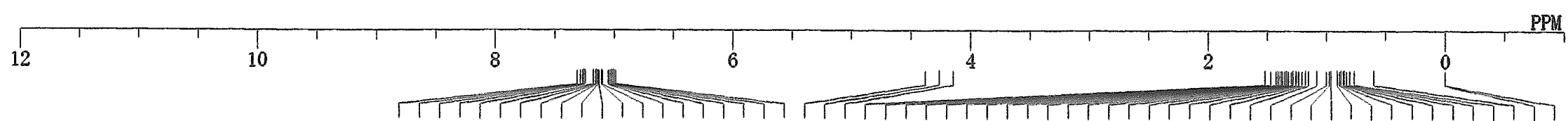

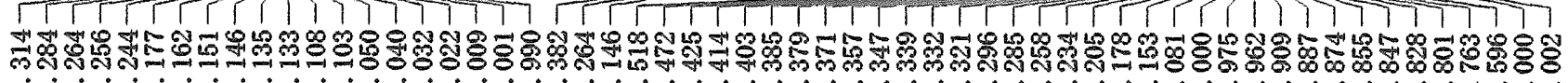

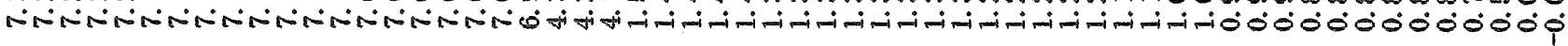




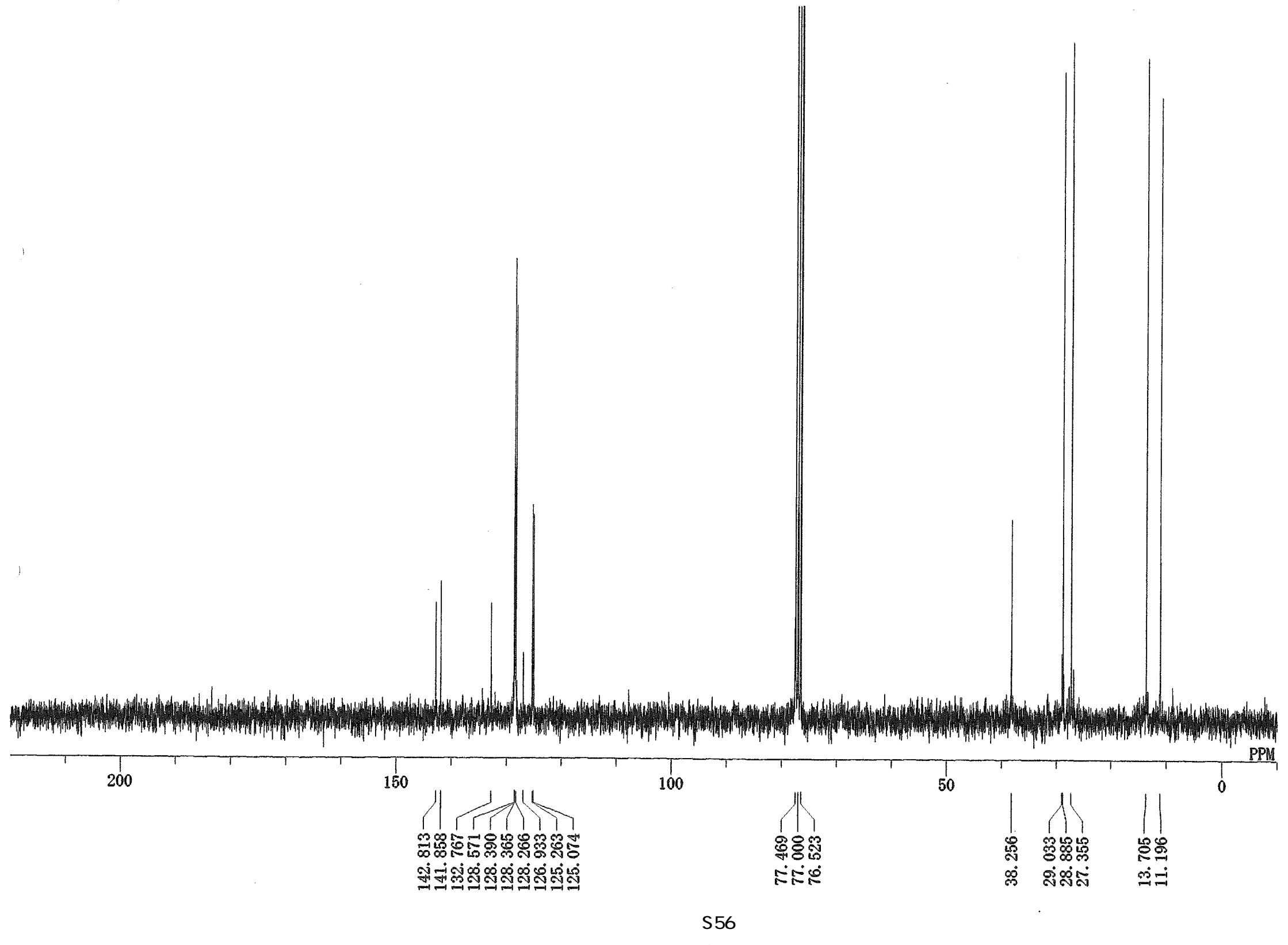




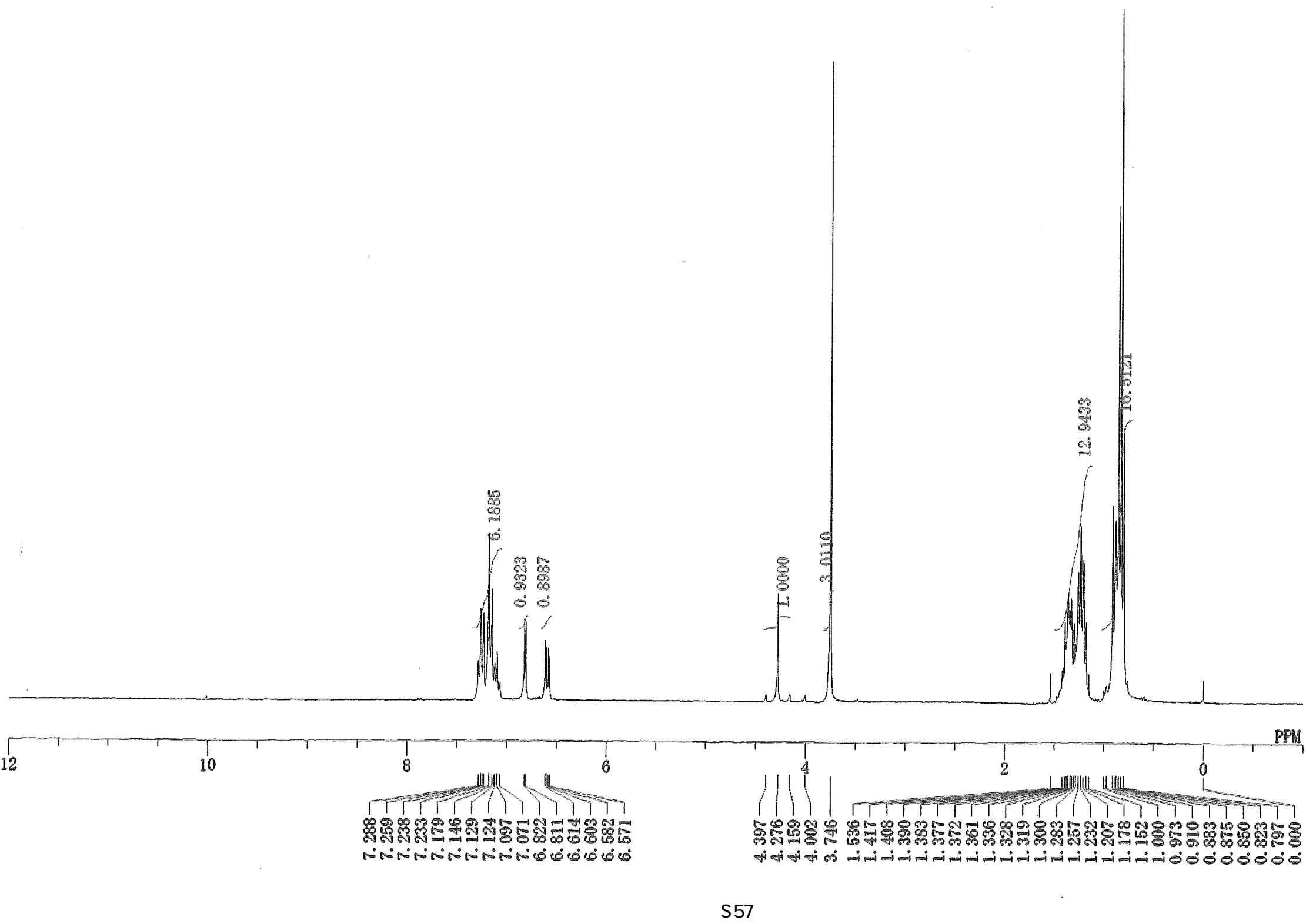




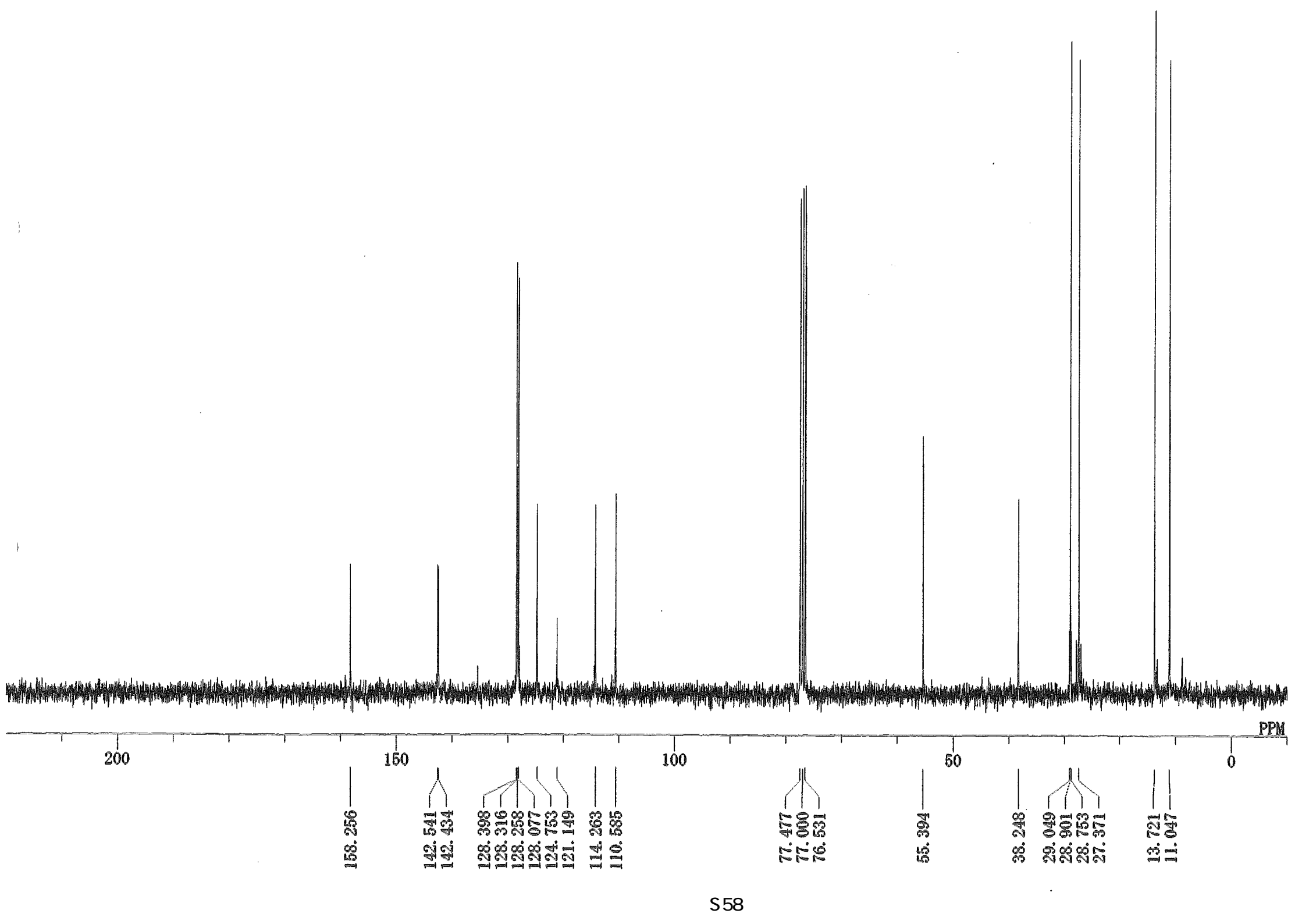




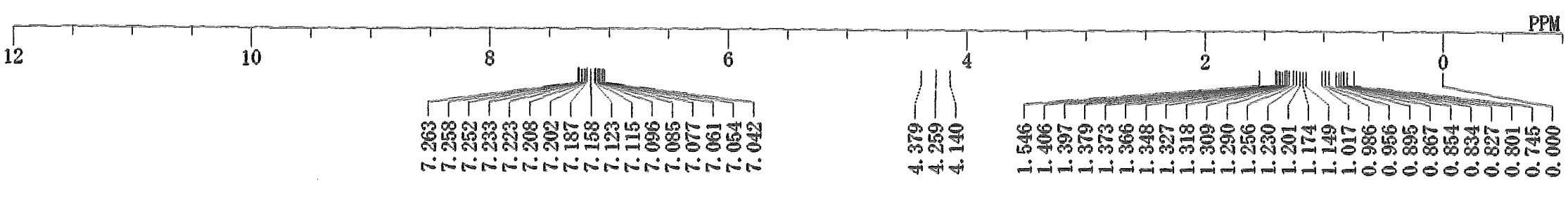


M2-OH-P120-13C

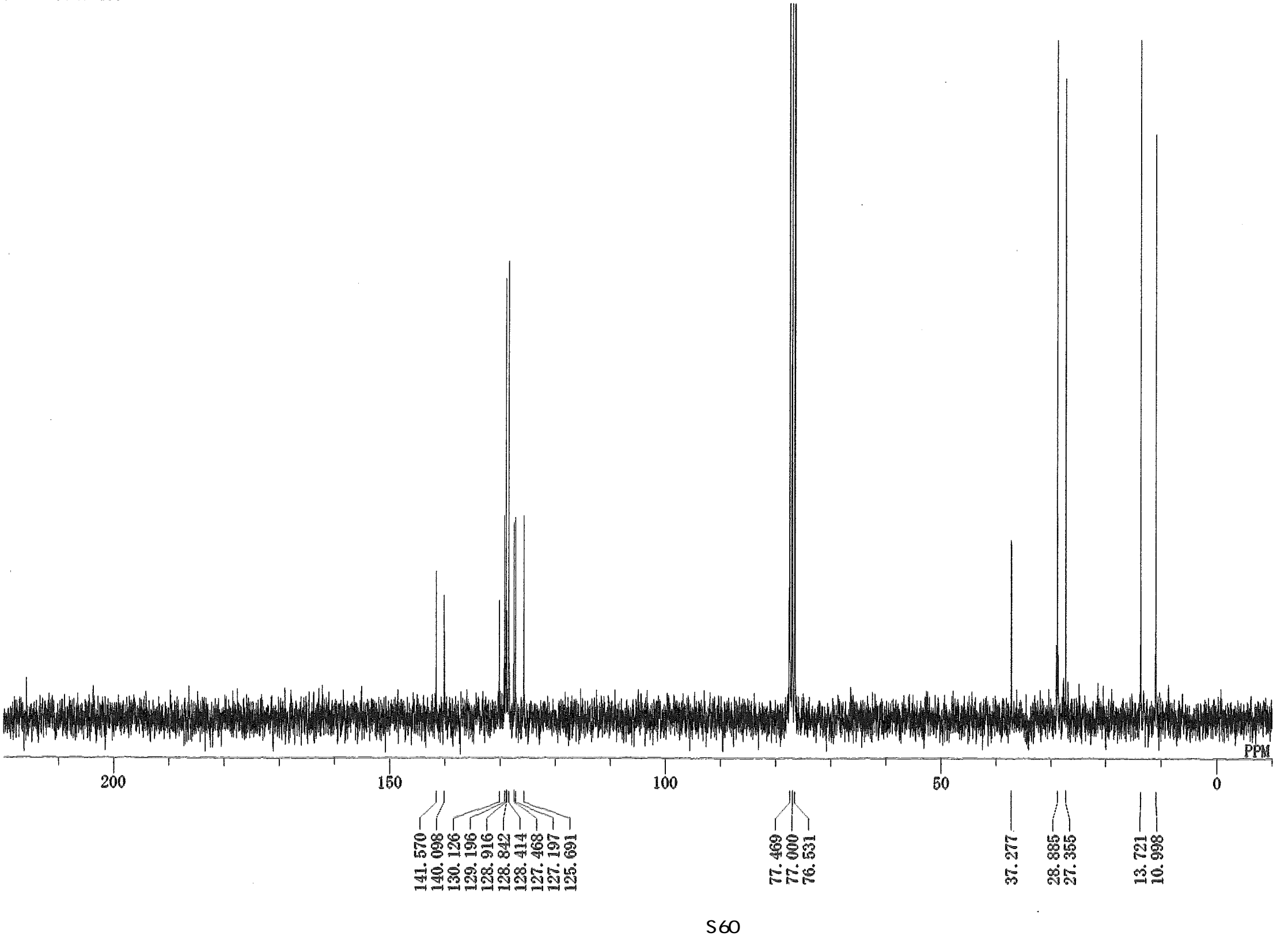

$0 \int_{n c s}^{\sin \mathrm{Bu}_{3}}$ 


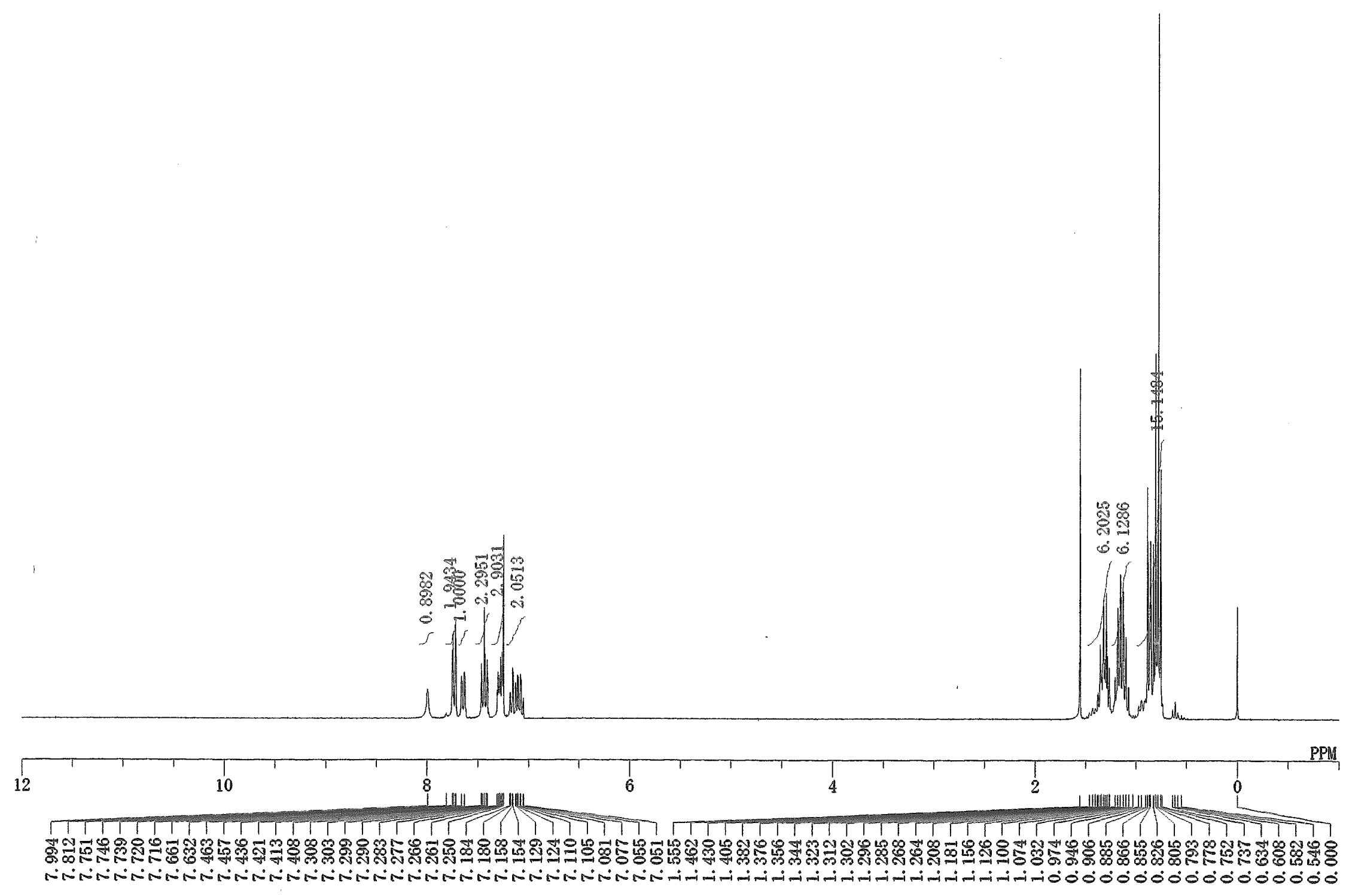


M2-0H-R71-13C

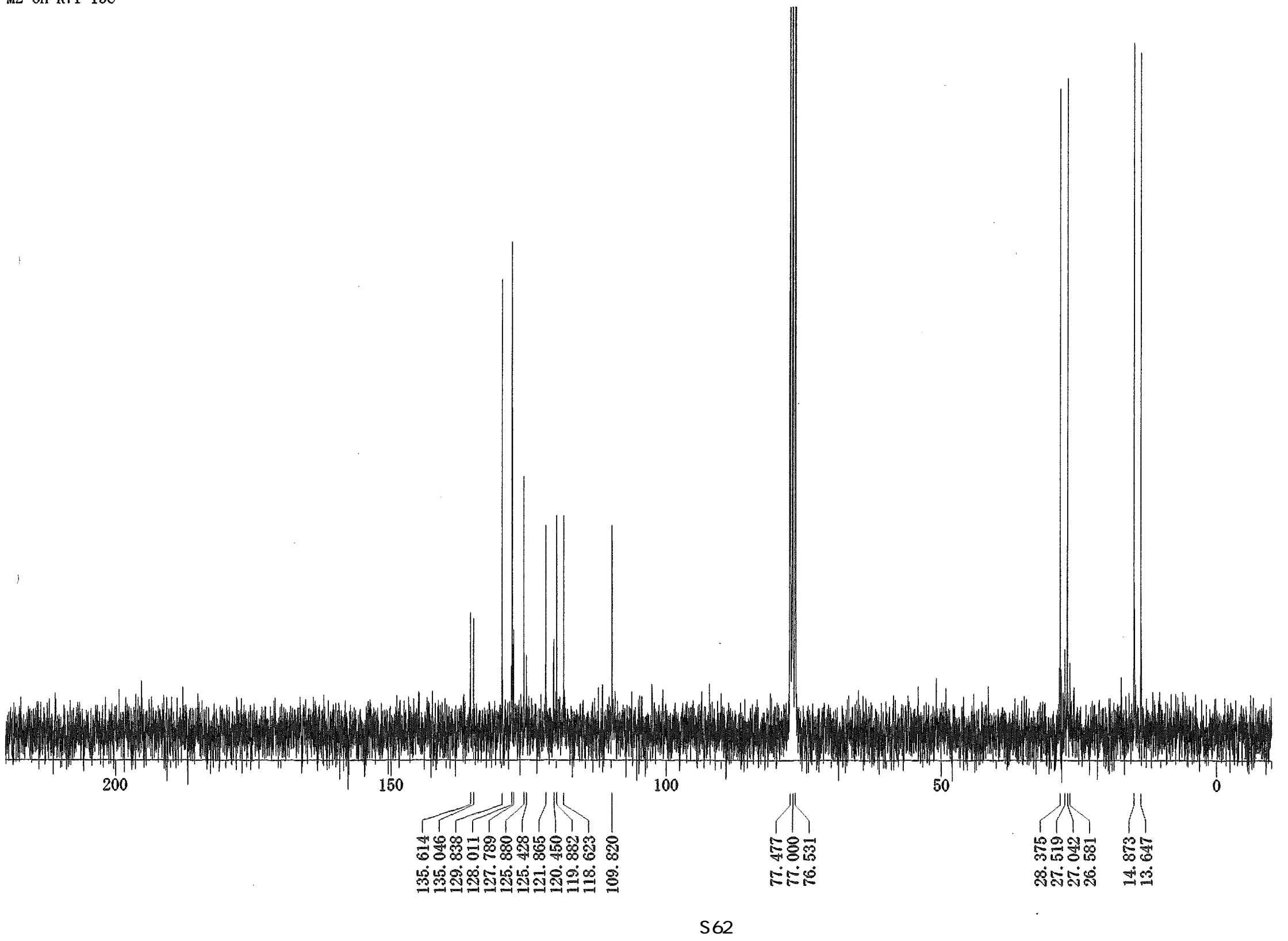

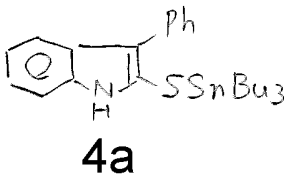




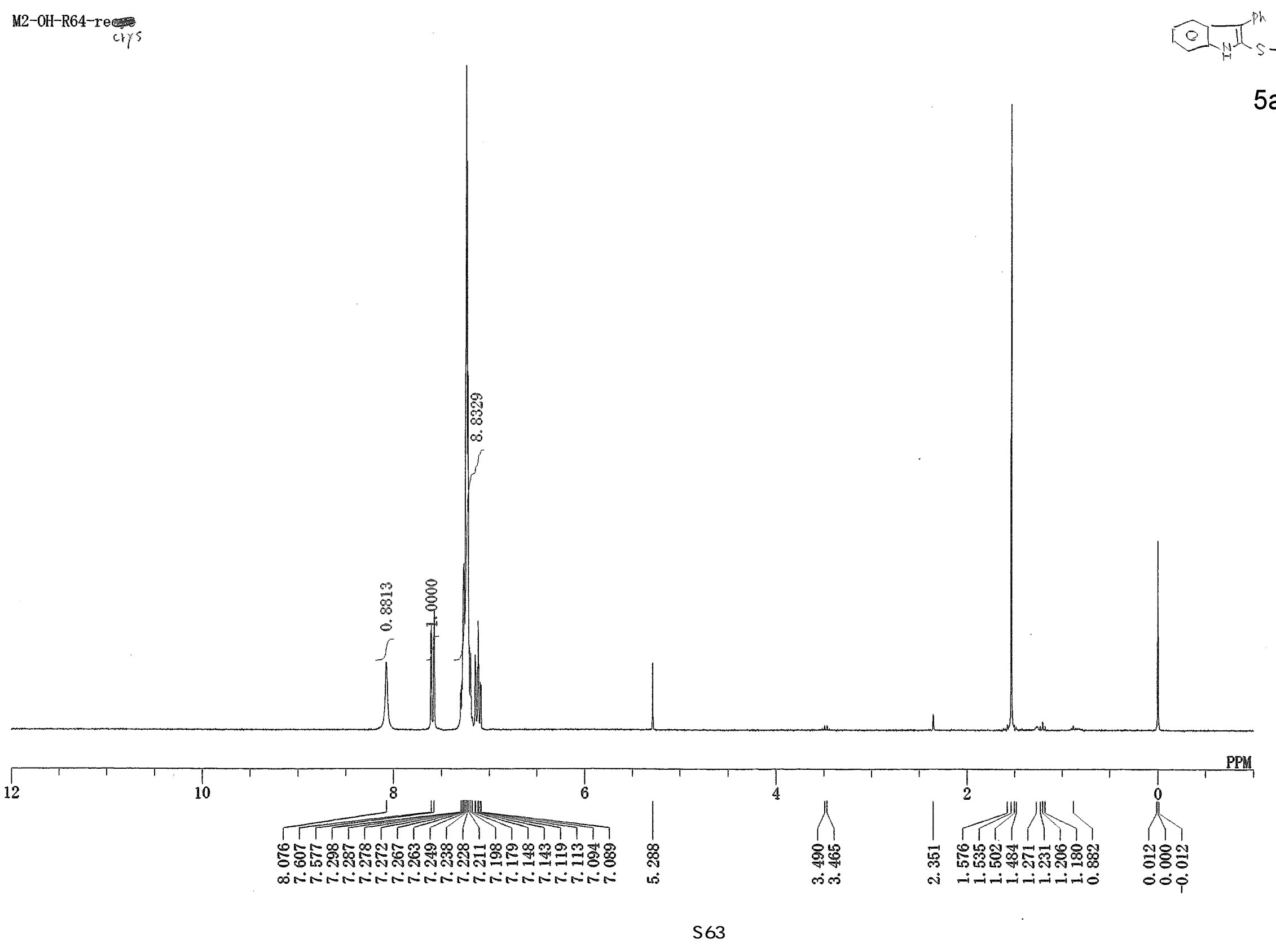


\Komanmr \personal \ota \reaction $\backslash \mathrm{R} 64 \backslash \mathrm{M} 2-\mathrm{OH}-\mathrm{R} 64-\mathrm{recyc}-13 \mathrm{C}$. als M2-OH-R64-rec $\frac{12 e-13 C}{45}$

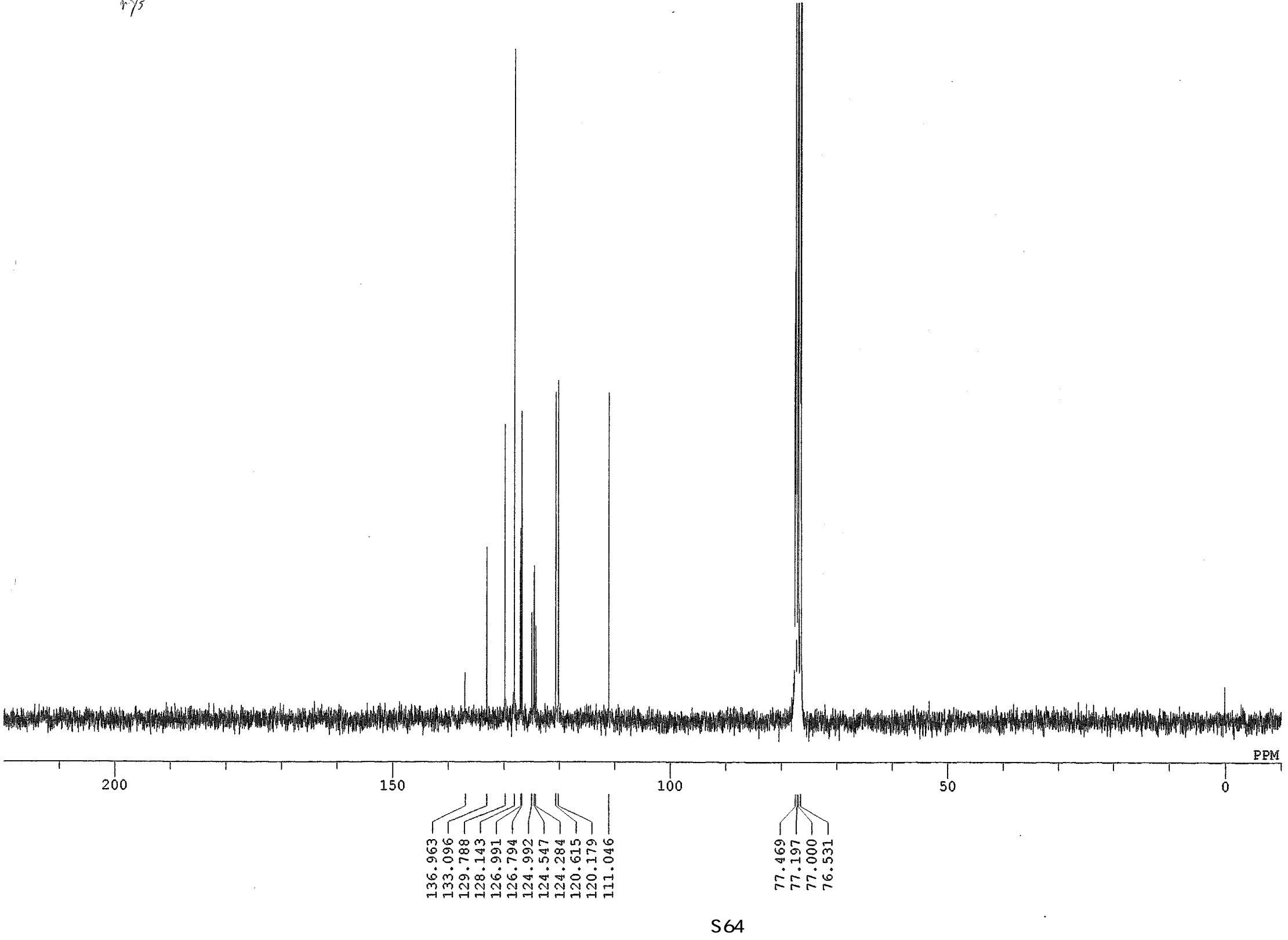




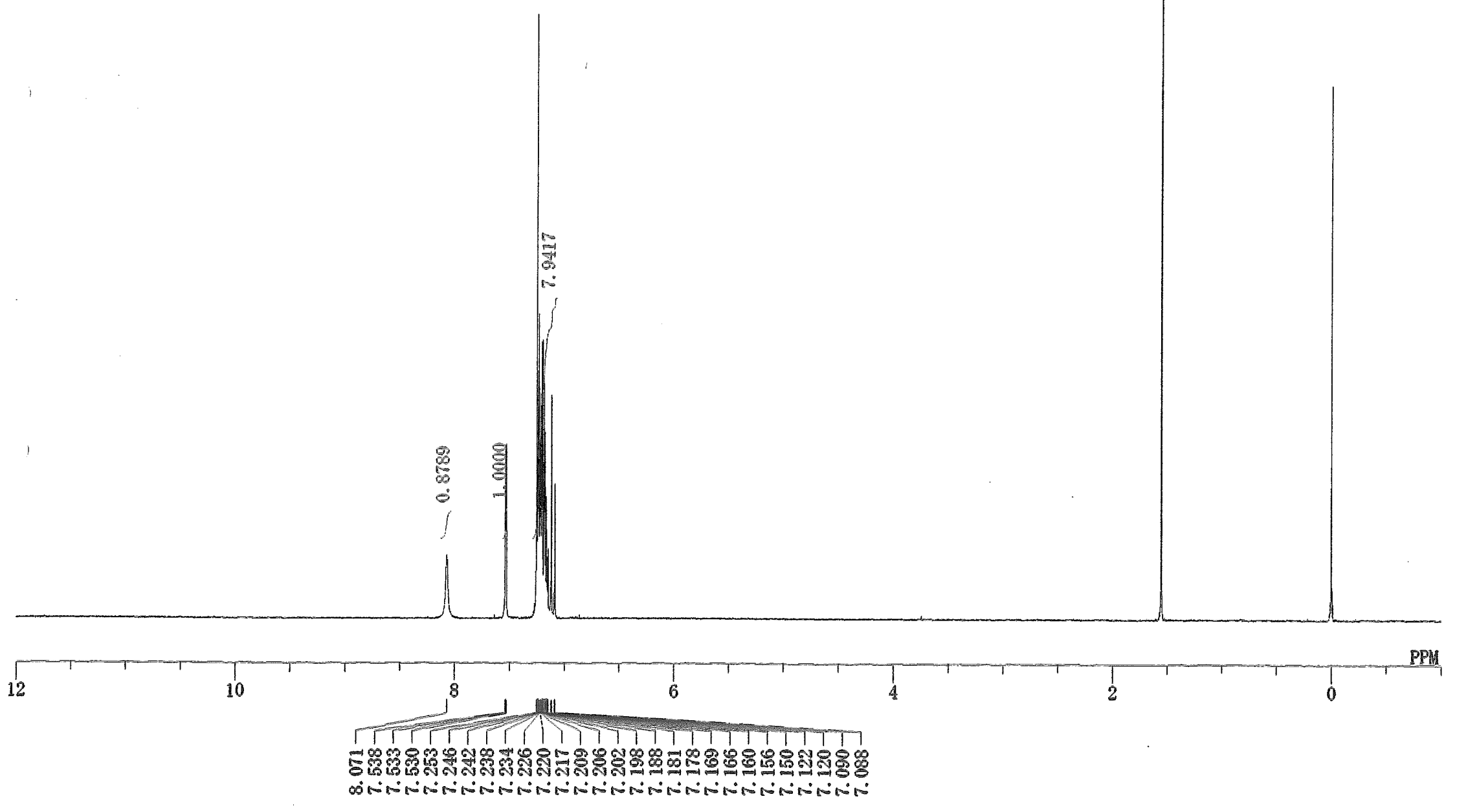


M2-OH-R82-13C

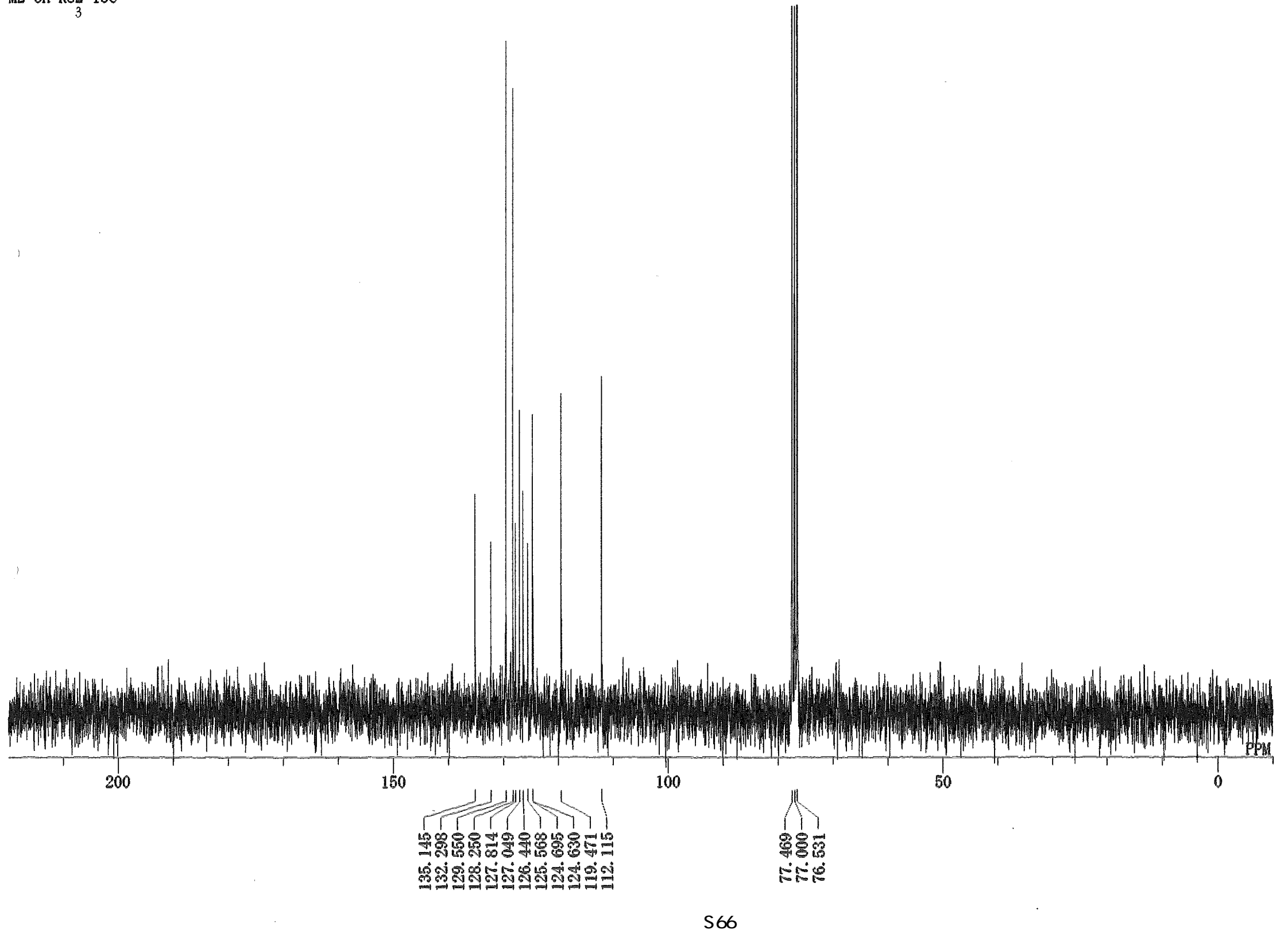

a

$5 b$ 
M2-OH-R85

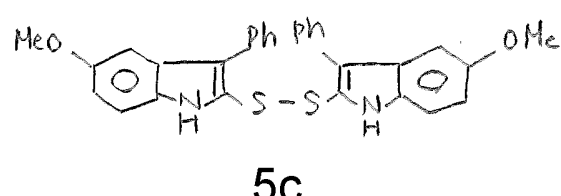

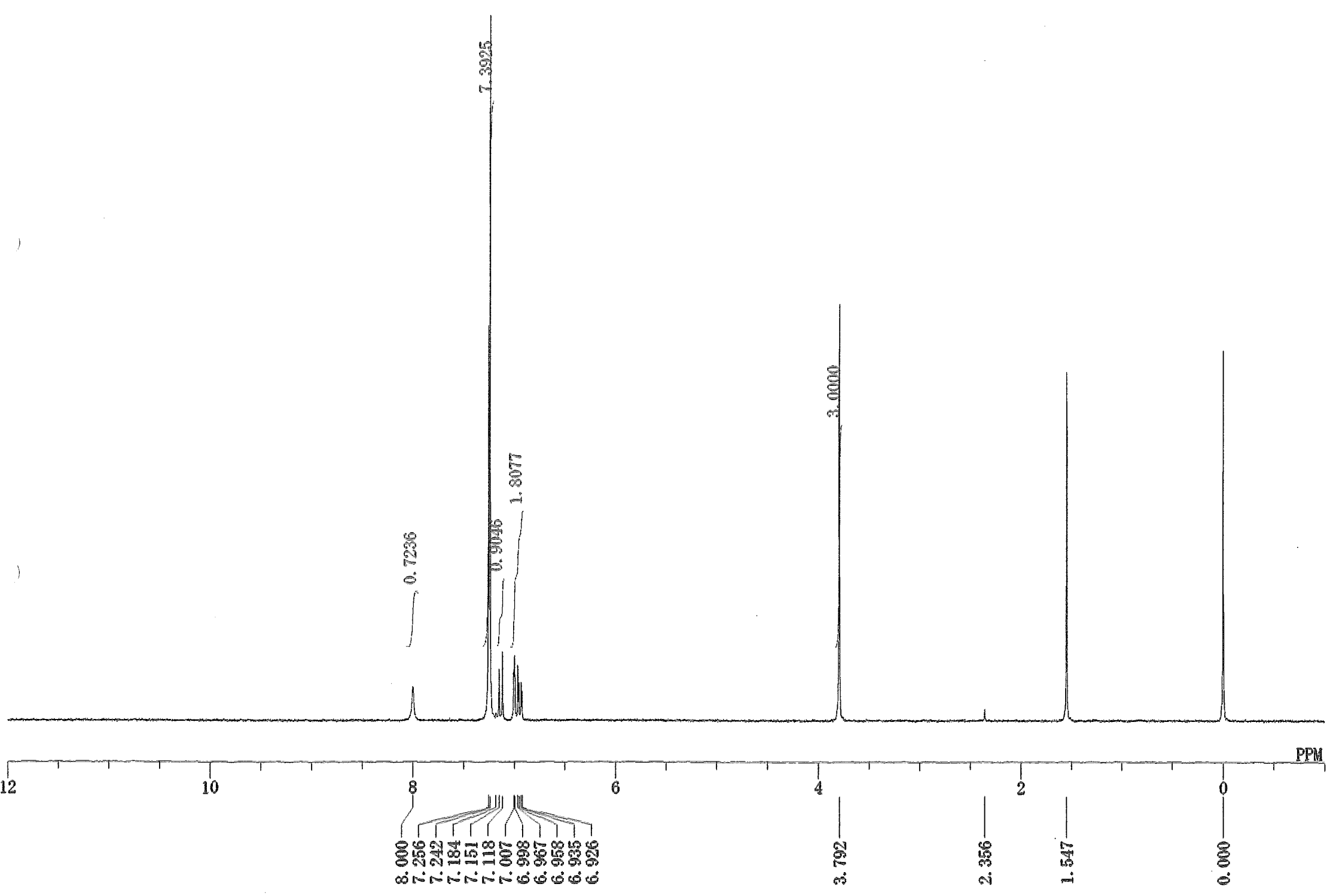


¥¥Komanmr $\mathrm{M} 2-\mathrm{OH}-\mathrm{R} 85-13 \mathrm{C}$

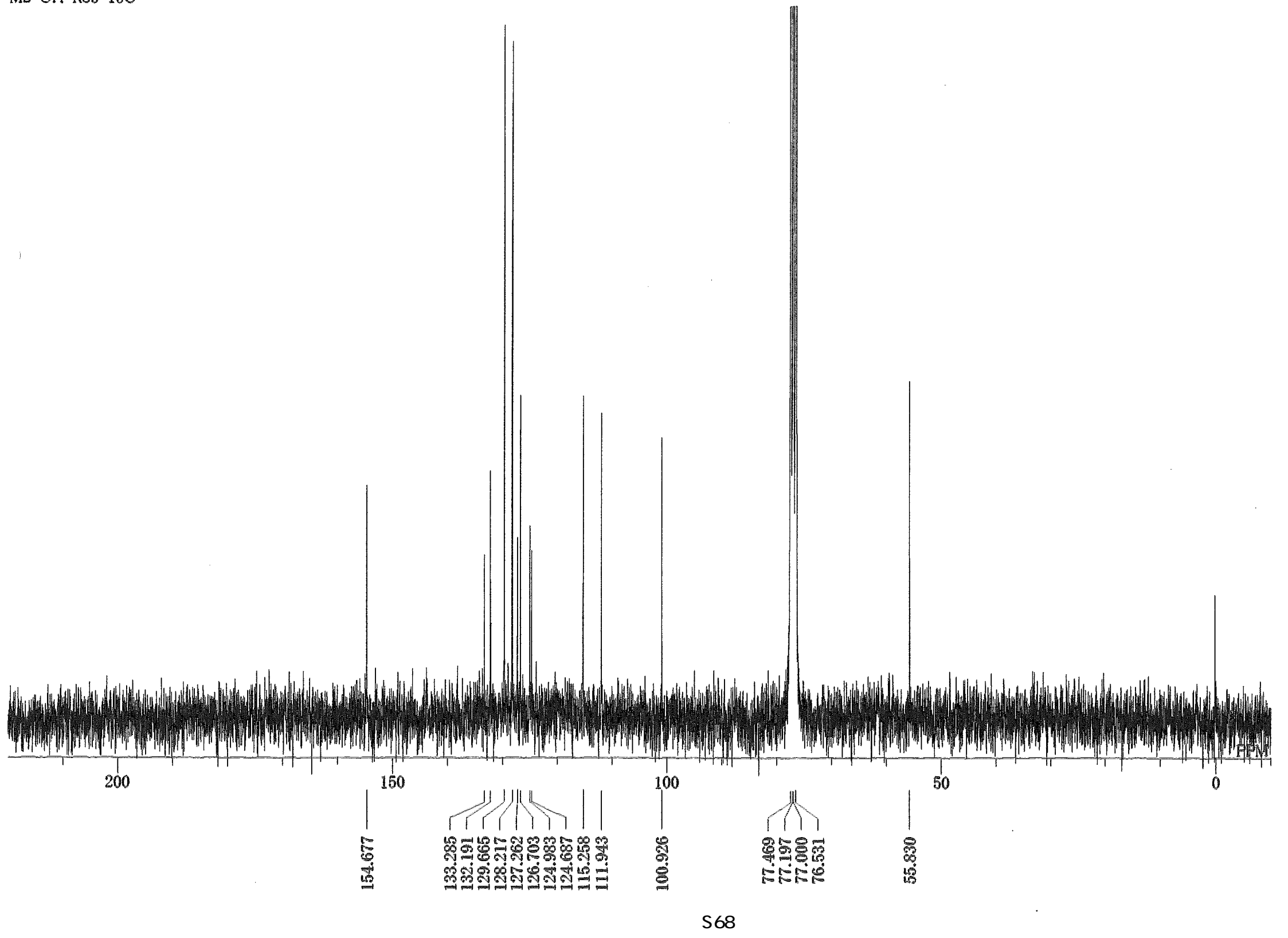

Her 
M2-0H-R87
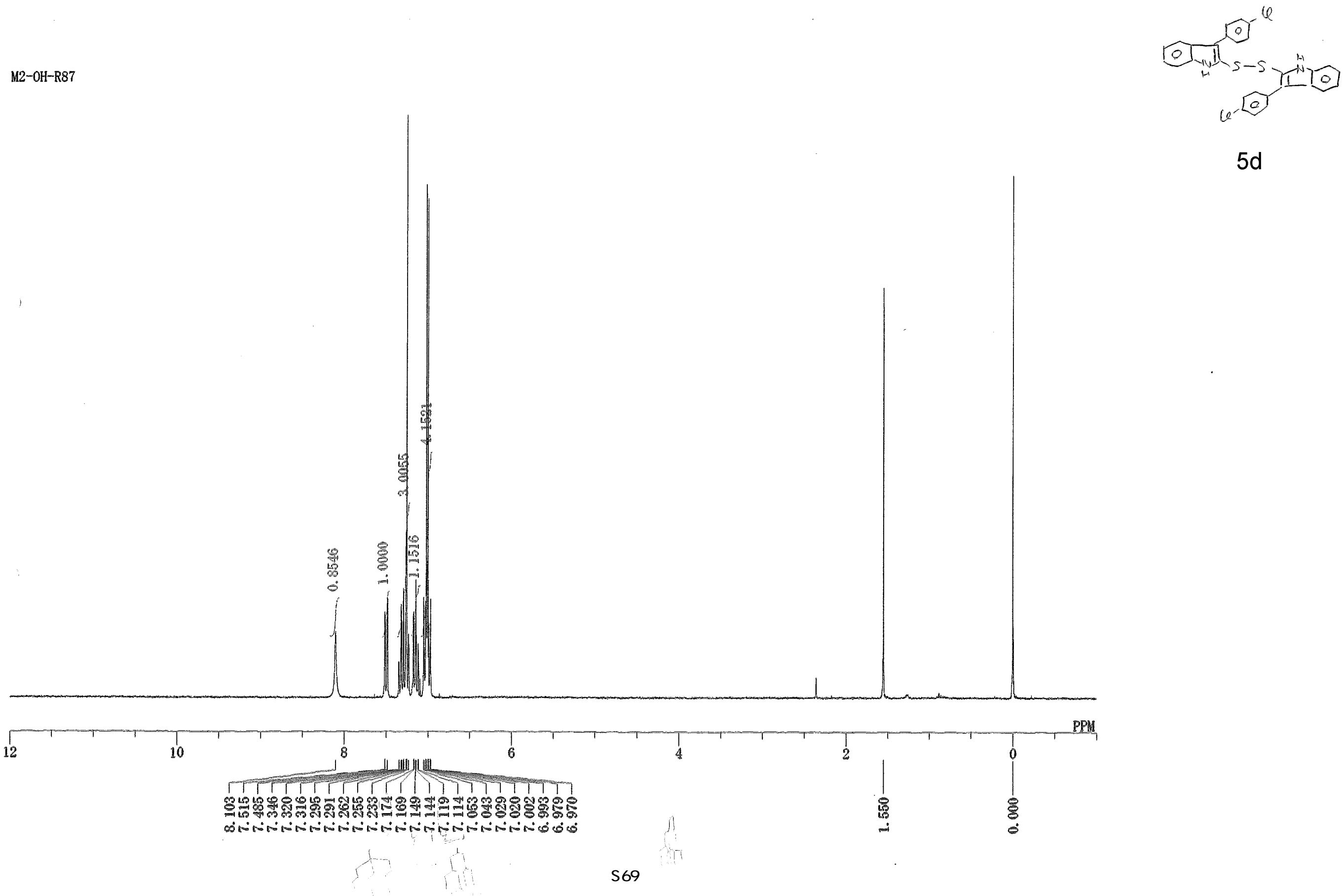
M2-OH-R87-13C

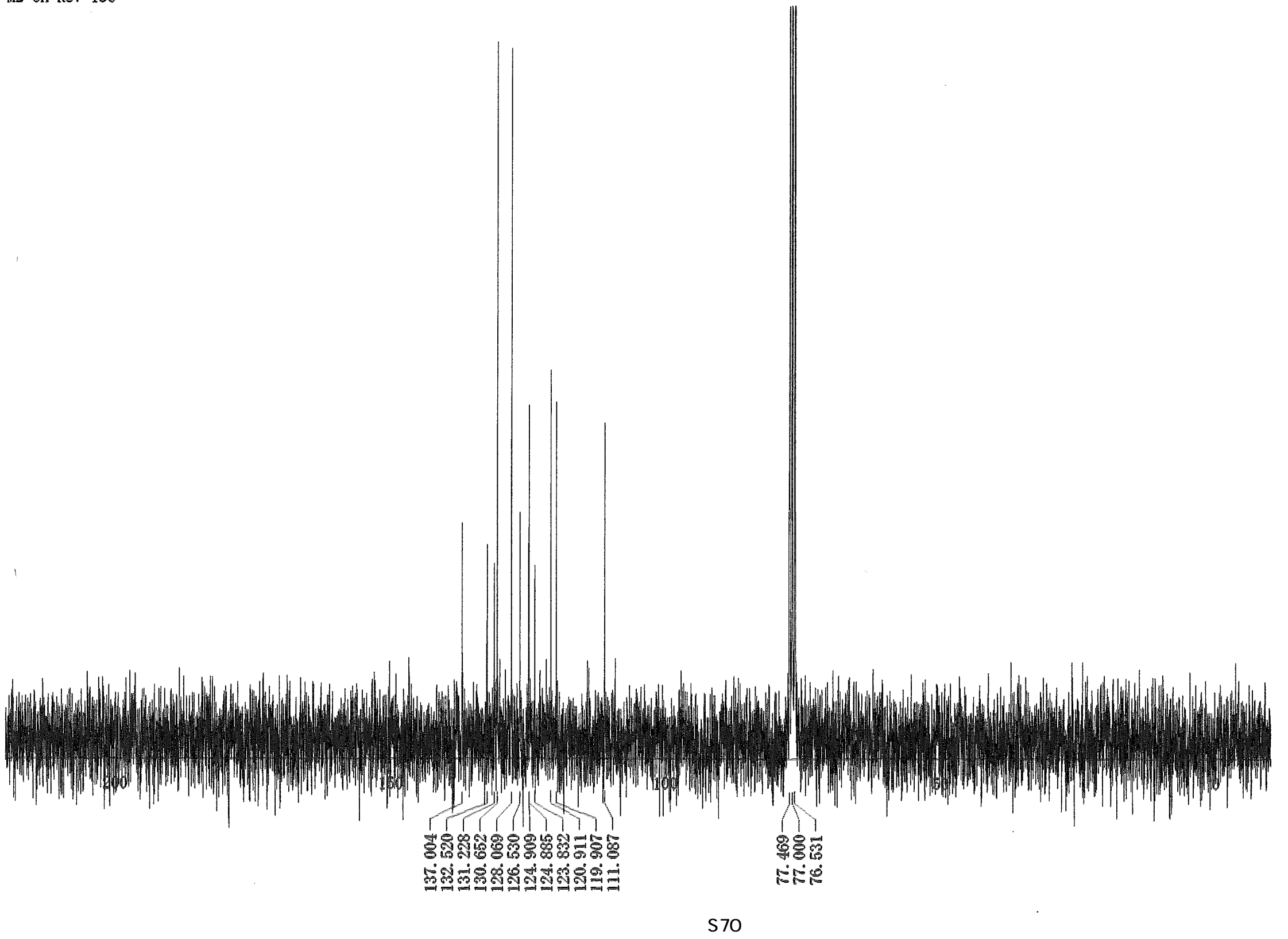

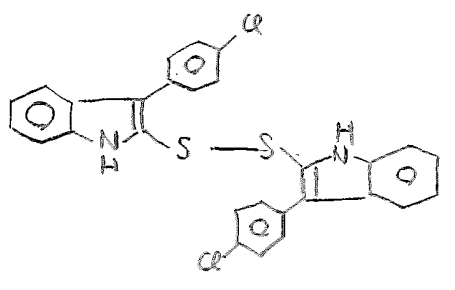

$5 d$ 


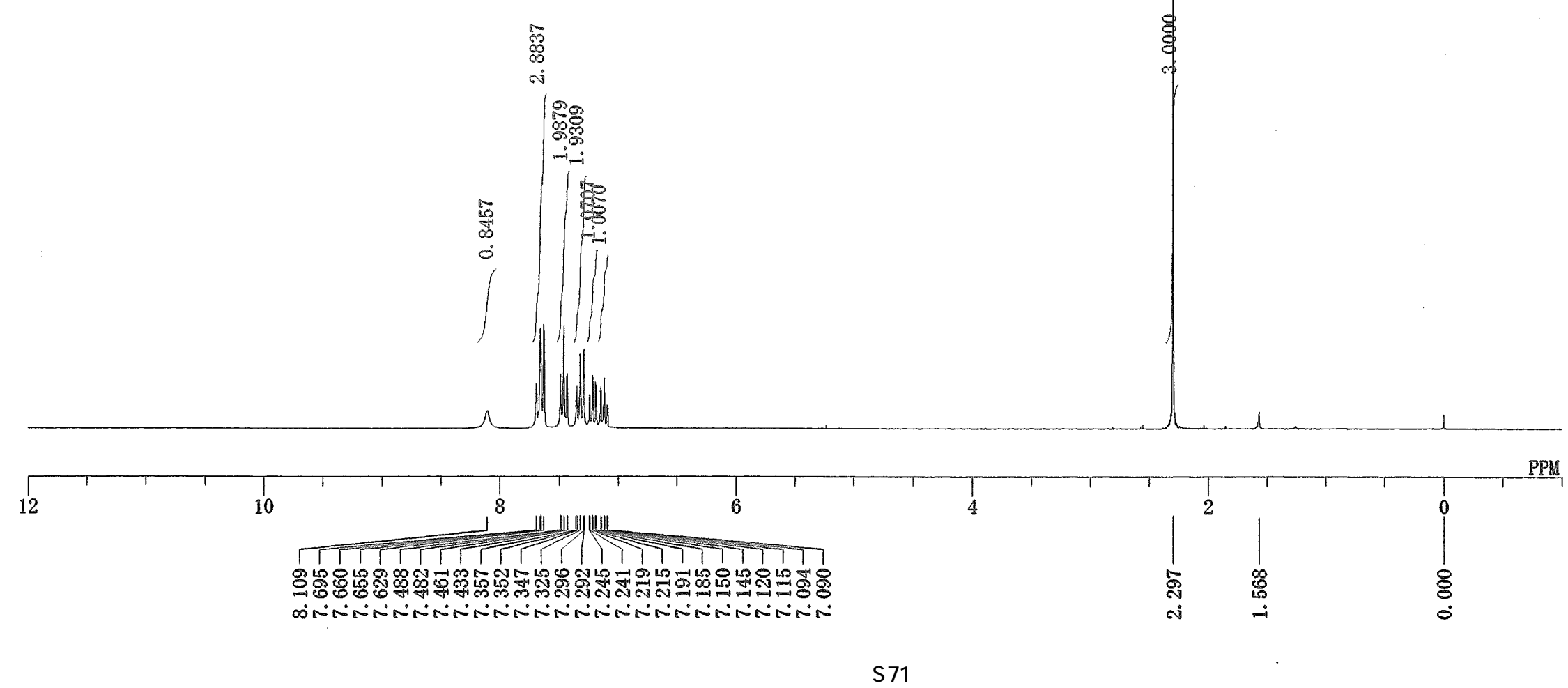




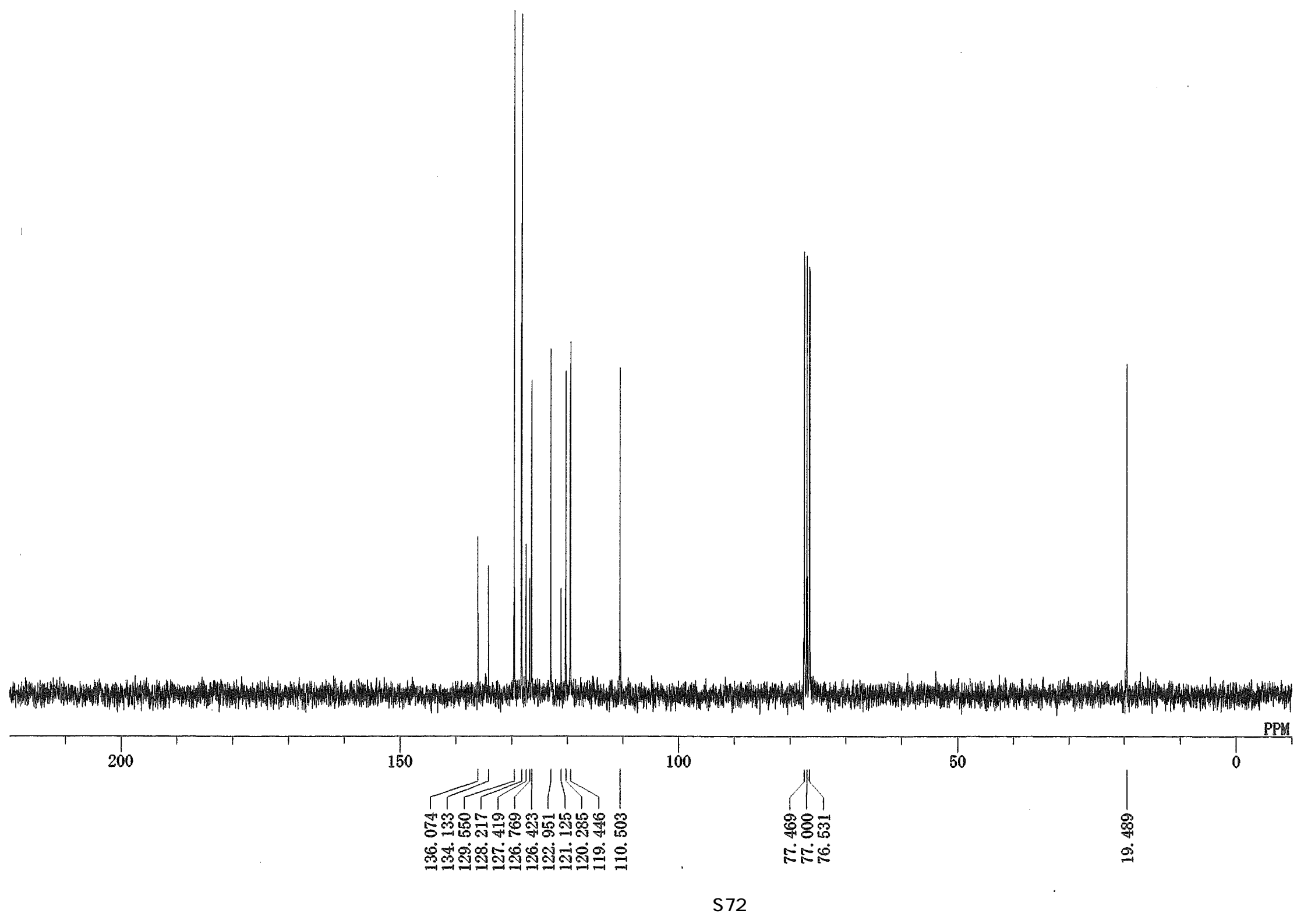


M2-OH-R90

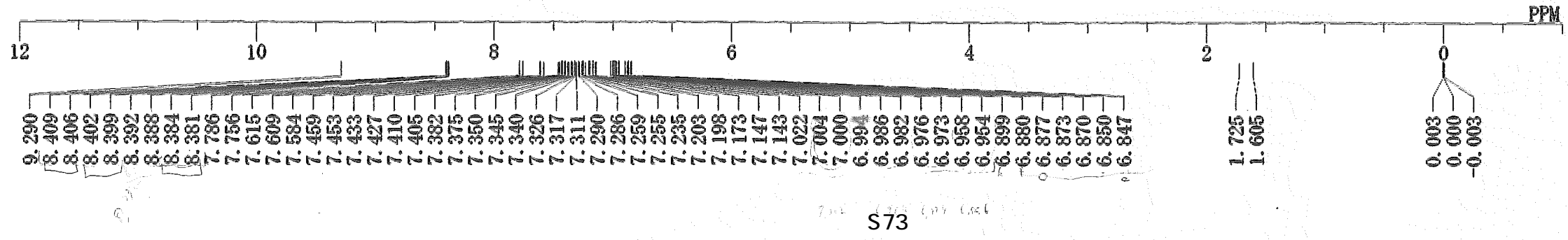




\section{M2-OH-R9O-13C}

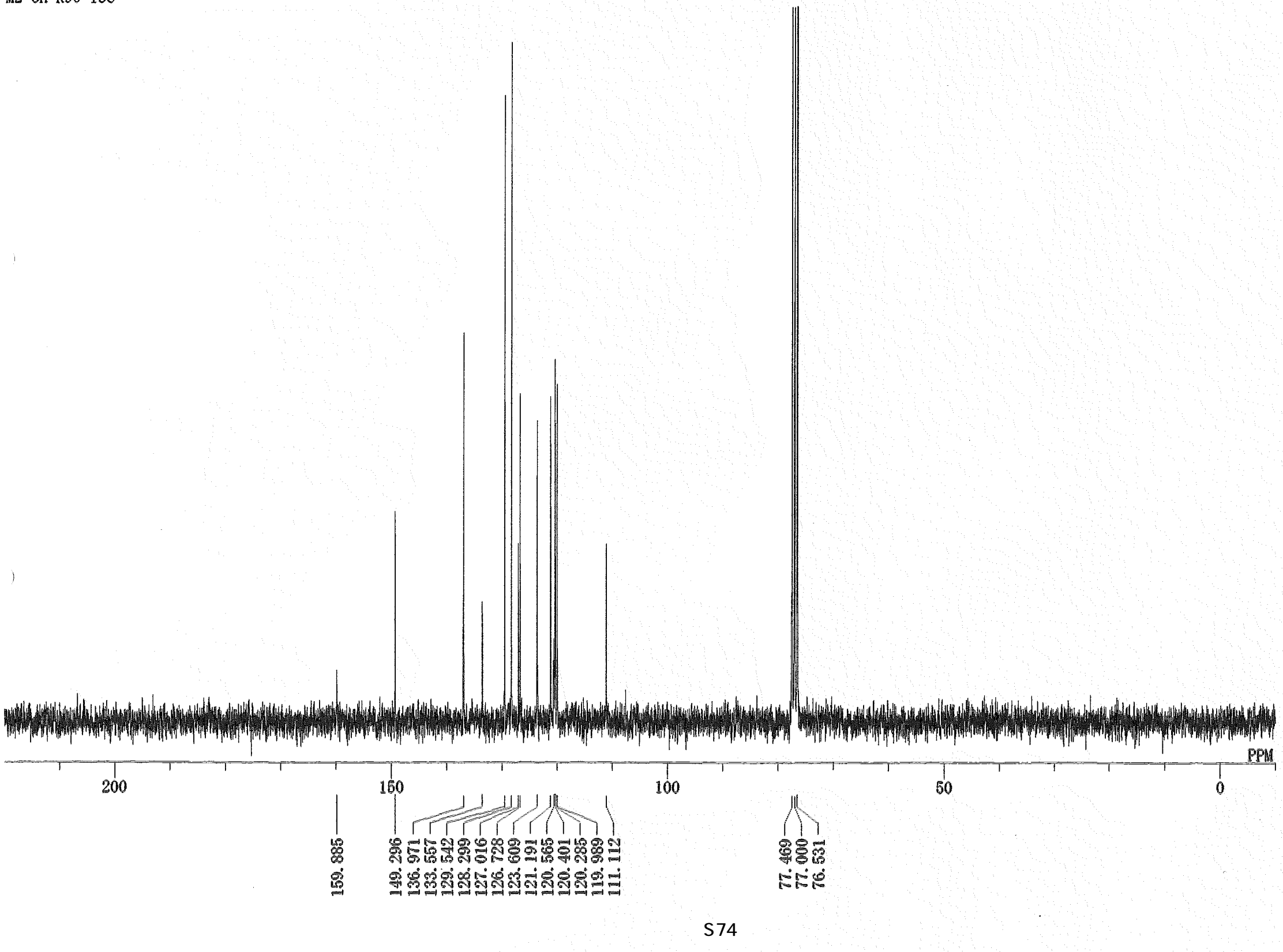

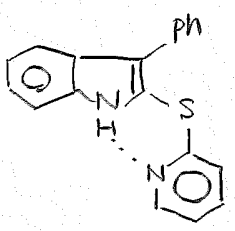

7 


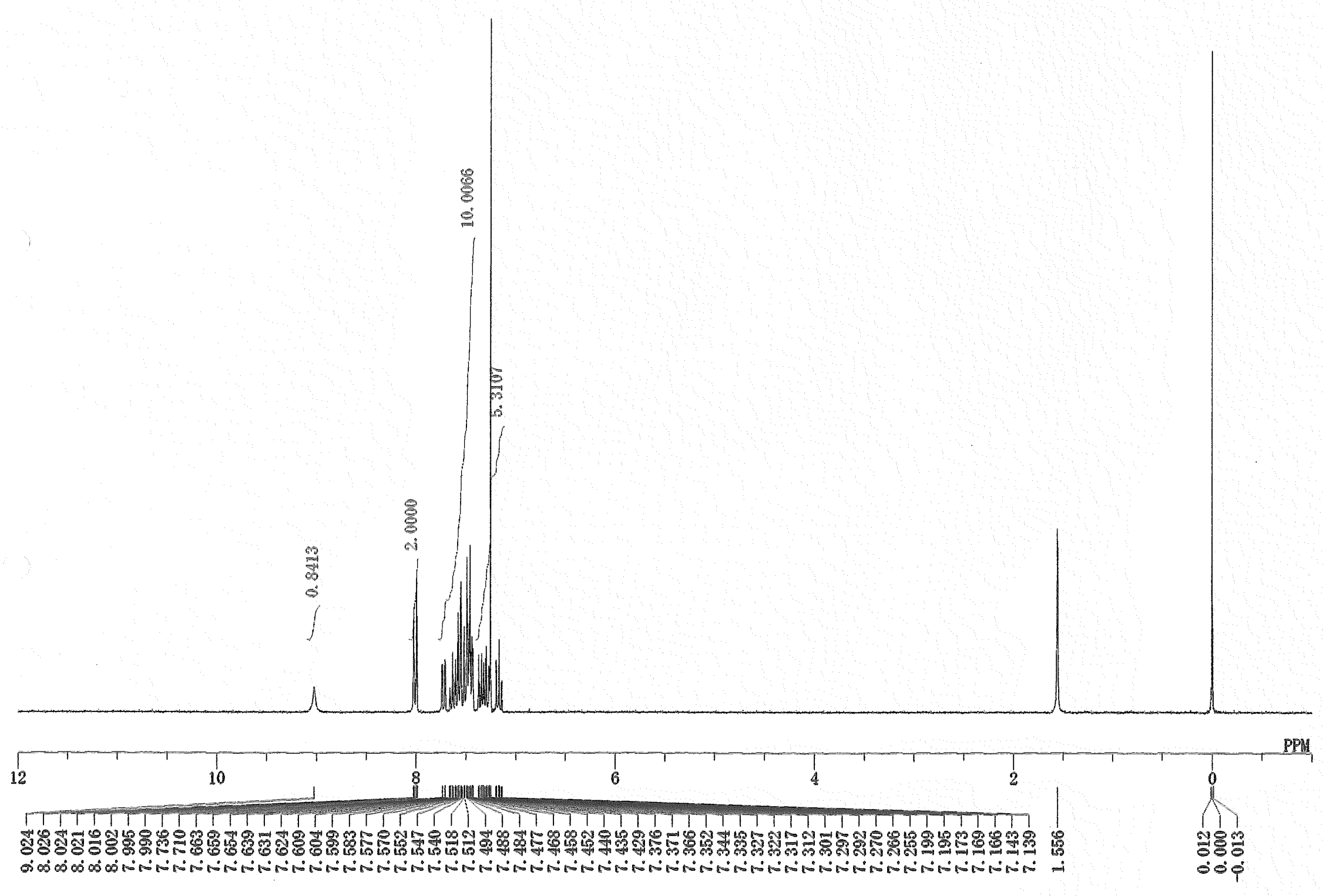




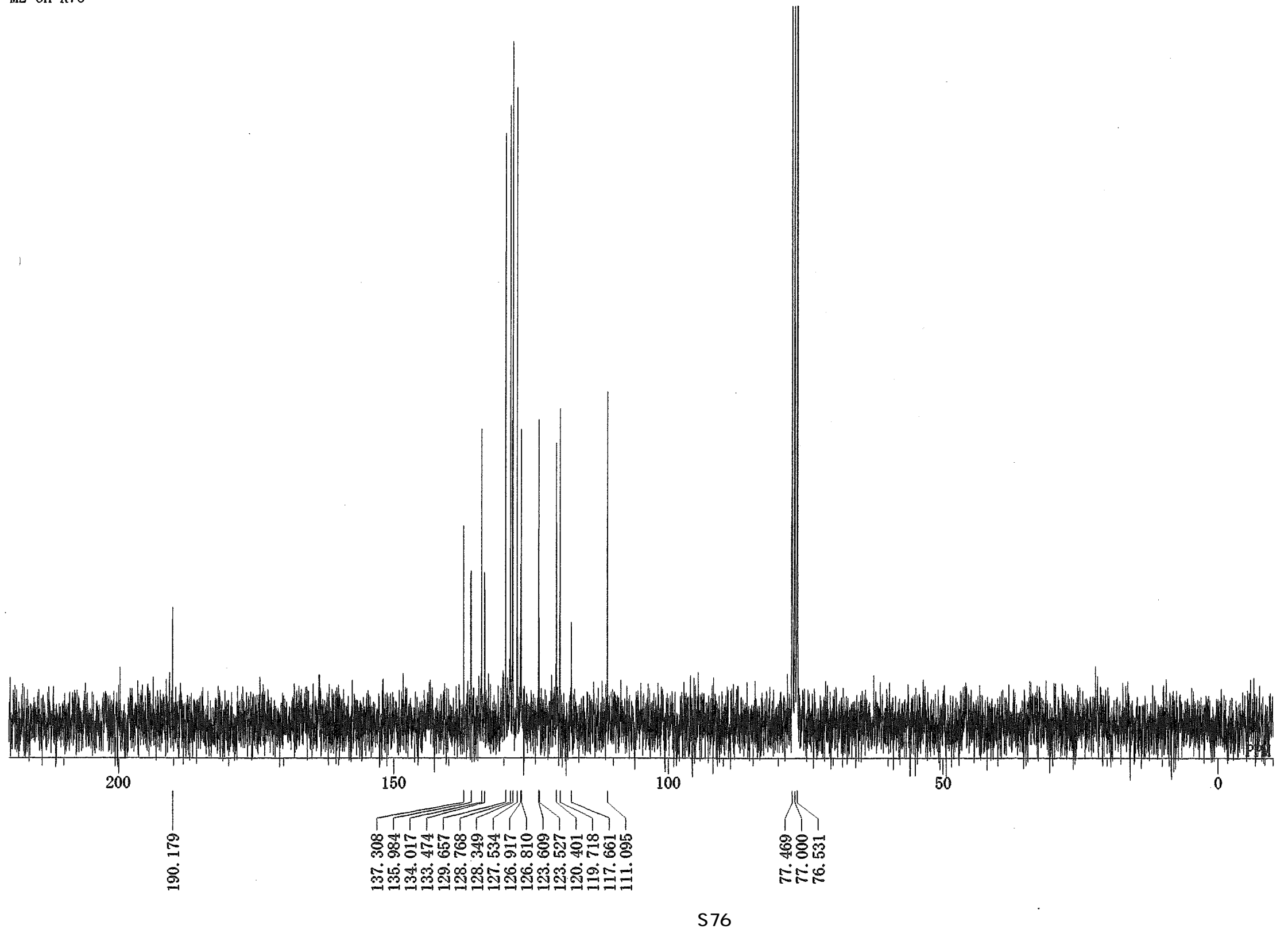

